\title{
Multi-omics of the gut microbial ecosystem in inflammatory bowel diseases
}

Jason Lloyd-Price ${ }^{1,2}$, Cesar Arze ${ }^{2}$, Ashwin N. Ananthakrishnan ${ }^{3}$, Melanie Schirmer ${ }^{1,3}$, Julian Avila-Pacheco ${ }^{4}$, Tiffany W. Poon ${ }^{1}$, Elizabeth Andrews ${ }^{3}$, Nadim J. Ajami ${ }^{5}$, Kevin S. Bonham ${ }^{1,2}$, Colin J. Brislawn ${ }^{6}$, David Casero ${ }^{7}$, Holly Courtney ${ }^{3}$, Antonio Gonzalez ${ }^{8}$, Thomas G. Graeber ${ }^{9}$, A. Brantley Hall ${ }^{1}$, Kathleen Lake ${ }^{10}$, Carol J. Landers ${ }^{11}$, Himel Mallick ${ }^{1,2}$, Damian R. Plichta ${ }^{1}$, Mahadev Prasad ${ }^{12}$, Gholamali Rahnavard ${ }^{1,2}$, Jenny Sauk ${ }^{13}$, Dmitry Shungin ${ }^{1,14}$, Yoshiki Vázquez-Baeza ${ }^{15,16}$, Richard A. White III ${ }^{6}$, IBDMDB Investigators ${ }^{17}$, Jonathan Braun ${ }^{7}$, Lee A. Denson ${ }^{10,18}$, Janet K. Jansson ${ }^{6}$, Rob Knight ${ }^{8,16,19}$, Subra Kugathasan ${ }^{12}$, Dermot P. B. McGovern ${ }^{11}$, Joseph F. Petrosino ${ }^{5}$, Thaddeus S. Stappenbeck ${ }^{20}$, Harland S. Winter ${ }^{21,22}$, Clary B. Clish ${ }^{4}$, Eric A. Franzosa ${ }^{2}$, Hera Vlamakis ${ }^{1}$, Ramnik J. Xavier ${ }^{1,3,23,24}$ \& Curtis Huttenhower ${ }^{1,2,24 *}$

Inflammatory bowel diseases, which include Crohn's disease and ulcerative colitis, affect several million individuals worldwide. Crohn's disease and ulcerative colitis are complex diseases that are heterogeneous at the clinical, immunological, molecular, genetic, and microbial levels. Individual contributing factors have been the focus of extensive research. As part of the Integrative Human Microbiome Project (HMP2 or iHMP), we followed 132 subjects for one year each to generate integrated longitudinal molecular profiles of host and microbial activity during disease (up to 24 time points each; in total 2,965 stool, biopsy, and blood specimens). Here we present the results, which provide a comprehensive view of functional dysbiosis in the gut microbiome during inflammatory bowel disease activity. We demonstrate a characteristic increase in facultative anaerobes at the expense of obligate anaerobes, as well as molecular disruptions in microbial transcription (for example, among clostridia), metabolite pools (acylcarnitines, bile acids, and short-chain fatty acids), and levels of antibodies in host serum. Periods of disease activity were also marked by increases in temporal variability, with characteristic taxonomic, functional, and biochemical shifts. Finally, integrative analysis identified microbial, biochemical, and host factors central to this dysregulation. The study's infrastructure resources, results, and data, which are available through the Inflammatory Bowel Disease Multi'omics Database (http://ibdmdb. org), provide the most comprehensive description to date of host and microbial activities in inflammatory bowel diseases.

Inflammatory bowel diseases (IBD) affect more than 3.5 million people, and their incidence is increasing worldwide ${ }^{1}$. These diseases, the most prevalent forms of which are Crohn's disease (CD) and ulcerative colitis (UC), are characterized by debilitating and chronic relapsing and remitting inflammation of the gastrointestinal tract (for CD) or the colon (in UC). These conditions result from a complex interplay among host ${ }^{2,3}$, microbial $^{4-6}$, and environmental ${ }^{7}$ factors. Drivers of IBD in the human genome include more than 200 risk variants, many of which are responsible for host-microbe interactions ${ }^{3}$. Common changes in the gut microbiome in individuals with IBD include an increase in facultative anaerobes, including Escherichia coli ${ }^{8}$, and a decrease in obligately anaerobic producers of short-chain fatty acids $(\mathrm{SCFAs})^{4}$. Here, to support a systems-level understanding of the aetiology of the IBD-associated gut microbiome that goes beyond previously reported metagenomic profiles, we introduce the IBDMDB, as part of the Integrative Human Microbiome Project.

We recruited 132 participants from five academic medical centres (three paediatric sub-cohorts: Cincinnati Children's Hospital,
Massachusetts General Hospital (MGH) Pediatrics, and Emory University Hospital; and two adult cohorts: MGH and CedarsSinai Medical Center; Fig. 1a, Extended Data Table 1, see Methods). Individuals not diagnosed with IBD on the basis of initial endoscopic and histopathologic findings were classified as 'non-IBD' controls. We analysed 651 biopsies (baseline) and 529 blood samples (approximately quarterly), which were collected in the clinic, and 1,785 stool samples, which were collected every two weeks using a home shipment protocol for one year (Fig. 1b). The latter yielded primarily microbially focused profiles: metagenomes (MGX), metatranscriptomes (MTX), proteomes (MPX), metabolomes (MBX), and viromes (VX) at several 'global' time points across all subjects (Fig. 1b), as well as denser, more intensive sampling from individuals with more variable disease activity (see Methods, Extended Data Fig. 1a-d). We generated multiple measurement types from many individual stool specimens, including 305 samples that yielded all stool-derived measurements, and 791 MGXMTX pairs (Fig. 1c, Extended Data Fig. 1b). Biopsies yielded hostand microbe-targeted human RNA sequencing (RNA-seq (HTX)),

\footnotetext{
${ }^{1}$ Infectious Disease and Microbiome Program, Broad Institute of MIT and Harvard, Cambridge, MA, USA. ${ }^{2}$ Department of Biostatistics, Harvard T. H. Chan School of Public Health, Boston, MA, USA. ${ }^{3}$ Gastroenterology, Massachusetts General Hospital, Boston, MA, USA. ${ }^{4}$ Metabolomics Platform, Broad Institute of MIT and Harvard, Cambridge, MA, USA. ${ }^{5}$ Molecular Virology and Microbiology, Baylor College of Medicine, Houston, TX, USA. ${ }^{6}$ Earth and Biological Sciences Directorate, Pacific Northwest National Lab, Richland, WA, USA. ${ }^{7}$ Department of Pathology and Laboratory Medicine,

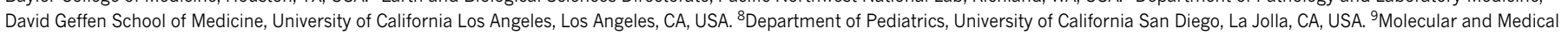
Pharmacology, University of California Los Angeles, Los Angeles, CA, USA. ${ }^{10}$ Department of Pediatrics, Cincinnati Children's Hospital Medical Center, Cincinnati, OH, USA. ${ }^{11} \mathrm{~F}$. Widjaja Foundation Inflammatory Bowel and Immunobiology Research Institute, Cedars-Sinai Medical Center, Los Angeles, CA, USA. ${ }^{12}$ Department of Pediatrics, Emory University, Atlanta, GA, USA. ${ }^{13}$ Vatche and Tamar Manoukian Division of Digestive Diseases, University of California Los Angeles, Los Angeles, CA, USA. ${ }^{14}$ Department of Odontology, Umeå University, Umeå, Sweden. ${ }^{15}$ Jacobs School of Engineering, University of California San Diego, La Jolla, CA, USA. ${ }^{16}$ Center for Microbiome Innovation, University of California San Diego, La Jolla, CA, USA. ${ }^{17} \mathrm{~A}$ list of participants and their affiliations appears at the end of the paper. ${ }^{18}$ Department of Pediatrics, University of Cincinnati College of Medicine, Cincinnati, OH, USA. ${ }^{19}$ Department of Computer Science and Engineering, University of California San Diego, La Jolla, CA, USA. ${ }^{20}$ Department of Pathology \& Immunology, Washington University, St. Louis, MO, USA. ${ }^{21}$ Department of Pediatrics, MassGeneral Hospital for Children, Boston, MA, USA. ${ }^{22}$ Department of Pediatrics, Harvard Medical School, Boston, MA, USA. ${ }^{23}$ Center for Microbiome Informatics and Therapeutics, Massachusetts Institute of Technology, Cambridge, MA, USA. ${ }^{24}$ These authors jointly supervised this work: Ramnik J. Xavier \& Curtis Huttenhower. *e-mail: chuttenh@hsph.harvard.edu
} 
a
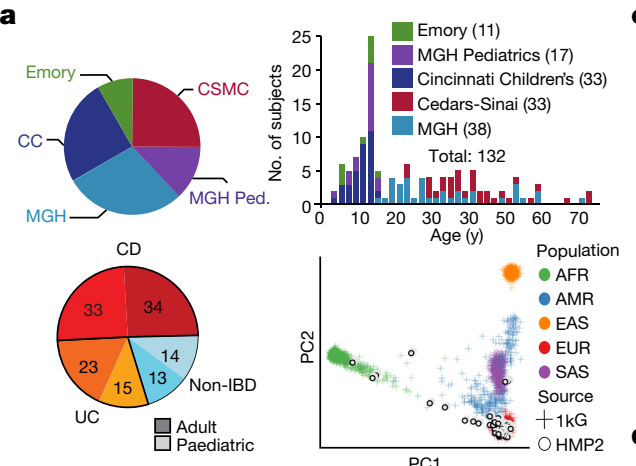

b
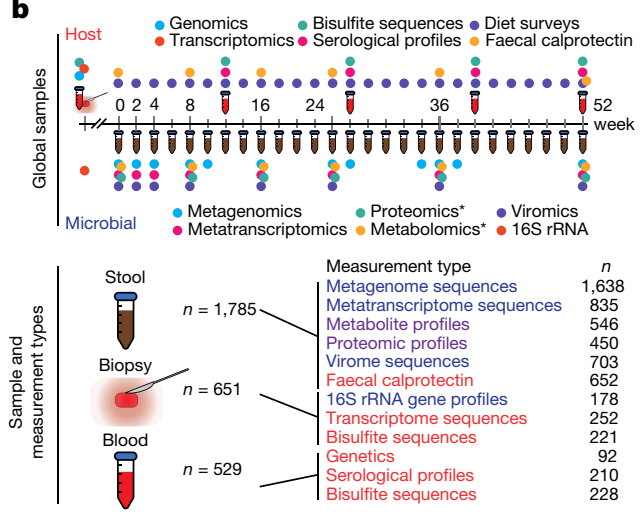

$n$
1,638
835
546
450
703
652
178
252
221
92
210
228

Fig. 1 Multi-omics of the IBD microbiome in the IBDMDB study. a, Overview of cohort characteristics. We followed 132 participants (with $\mathrm{CD}$, with UC, or without IBD (control)) for one year each. Principal component analysis (PCA) of SNP profiles shows that the resulting IBDMDB cohort is mostly of European ancestry as compared to the 1000 Genomes ( $1 \mathrm{kG})$ reference (see Methods). b, Sampling strategy. The study yielded host and microbial data from colon biopsy (baseline), blood (approximately quarterly), and stool (every two weeks), assessing global time points for all subjects and dense time courses for a subset. Raw, nonquality-controlled sample counts are shown. c, Overlap of multi-omic measurements from the same sample (strict) or from near-concordant time points (with differences of up to 2 or 4 weeks; see Methods). d, Principal coordinates analysis (PCoA) based on species-level BrayCurtis dissimilarity; most variation is driven by a tradeoff between phylum Bacteroidetes versus Firmicutes. Samples from individuals with IBD (CD in particular) had weakly lower Gini-Simpson alpha diversity

epigenetic reduced representation bisulfite sequencing (RRBS), and $16 S$ rRNA gene amplicon sequencing (16S), which were matched with human exome sequencing, serological profiles, and RRBS from blood. All data are available at https://ibdmdb.org/.

\section{Multi-omic gut microbiome changes in IBD}

Consistent with prior studies ${ }^{4,5}$, although subsets of IBD (CD in particular) contributed to the second axis of taxonomy-based principal coordinates (Fig. 1d, Extended Data Fig. 2a), inter-individual variation accounted for the majority of variance for all measurement types ${ }^{5,9,10}$ (Fig. 1e, f, Extended Data Fig. 2a). Even relatively large effects, such as disease status or physiological and technical factors, explained a smaller proportion of variation (Fig. 1f); this was true across measurement types, although these captured distinct aspects of IBD dysbiosis (see below).

Most measurement types captured correlated changes among and within subjects, cross-sectionally and longitudinally (Fig. 1e). Functional profiles, measured from MGX, MTX, and MPX, were the most tightly coupled (Fig. 1e), although some individual featurewise correlations were weak (Spearman's correlation MGX-MTX $0.44 \pm 0.10$ (mean \pm s.d.), MGX-MPX $0.14 \pm 0.083$, and MTXMPX $0.18 \pm 0.0961$; Extended Data Fig. 2b). Unexpectedly, characterized enzymes tended to be only weakly correlated with their known

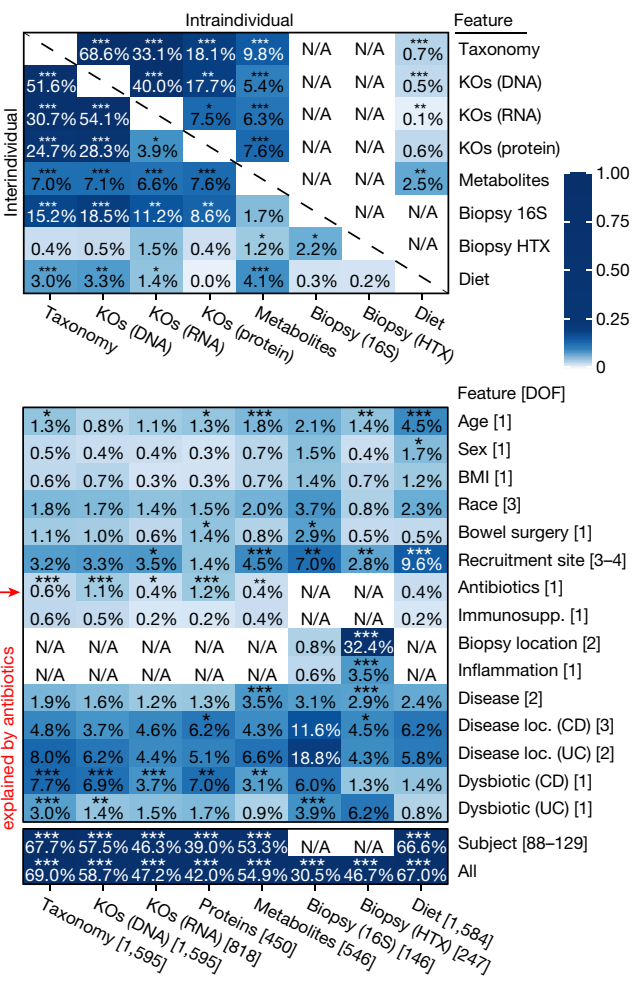

(Wald test $P=0.26$ and 0.014 for UC and CD compared with non-IBD, respectively). e, Mantel tests quantifying variance explained (square of Mantel statistic) between measurement type pairs, with differences across subjects (inter-individual) or within subjects over time (intra-individual; see Methods); results show tight coupling across measurement types. Sample sizes in f. f, PERMANOVA shows that inter-individual variation is largest for all measurement types, with even relatively large effects (for example, antibiotics or IBD phenotype) capturing less variation (see Methods). Stratified tests (CD/UC) consider only samples within the indicated phenotype (note that sample counts decrease for these, resulting in larger expected covariation by chance). Stars show FDR-corrected statistical significance (FDR $* P \leq 0.05, * * P \leq 0.01$, *** $P \leq 0.001$ ). Variance is estimated for each feature independently (Methods). 'All' refers to a model with all metadata. Total $n$ for each measurement type is shown in square brackets, distributed across up to 132 subjects (Extended Data Fig. 1a, see Methods).

substrates or products (Supplementary Fig. 1). Although our dietary characterization was obtained through a very broad-level food frequency questionnaire, it provides an initial characterization of longitudinal diet-microbiome coupling in a substantial population over many months; diet accounted for a small but significant 3\% (false discovery rate (FDR) $P=7.4 \times 10^{-4}$ ) of taxonomic variation between subjects, and $0.7 \%\left(\right.$ FDR $\left.P=4.3 \times 10^{-4}\right)$ of variation longitudinally.

Simple cross-sectional differences between individuals with IBD and those without (Supplementary Tables 1-14) were most apparent in the metabolome (Figs. 1f, 2a, Extended Data Fig. 2a, c, d, see Methods). Overall, metabolite pools were less diverse in individuals with IBD, paralleling previous observations for microbial diversity (Supplementary Table 2); this might be caused by poor nutrient absorption, greater water or blood content in the bowels, and shorter bowel transit times in individuals with active $\mathrm{IBD}^{11}$. The smaller number of compounds that were more abundant in patients with IBD included polyunsaturated fatty acids such as adrenate and arachidonate. Pantothenate and nicotinate (vitamins B5 and B3, respectively) were particularly depleted in the gut during IBD; this is notable because these are not typically among the $B$ vitamins that are deficient in the serum of patients with $\mathrm{IBD}^{12}$, although low nicotinate levels have been detected during active $\mathrm{CD}^{13}$. Both vitamins are required to produce cofactors used in lipid metabolism ${ }^{14}$, and nicotinate has anti-inflammatory and anti-apoptotic 
a

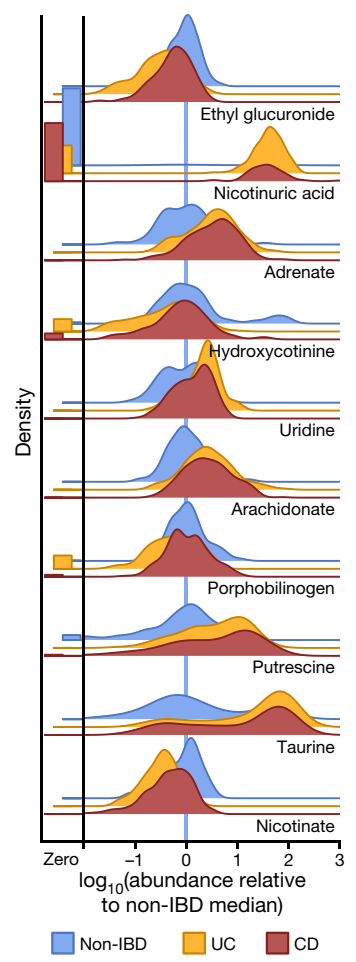

b 10

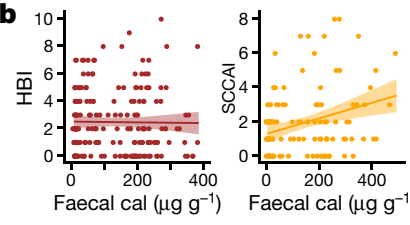

c
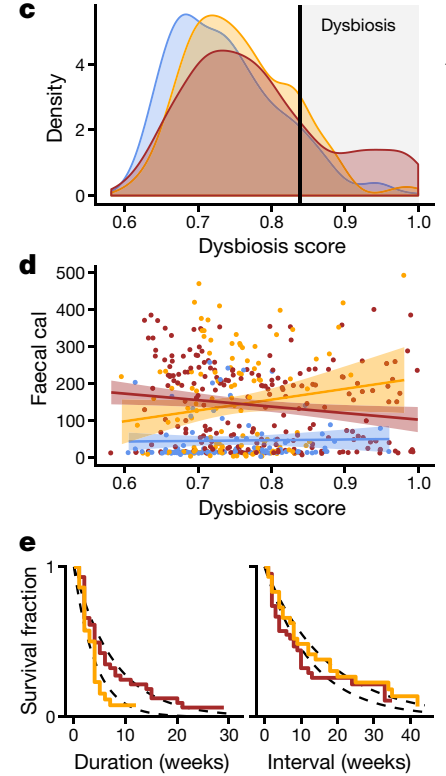
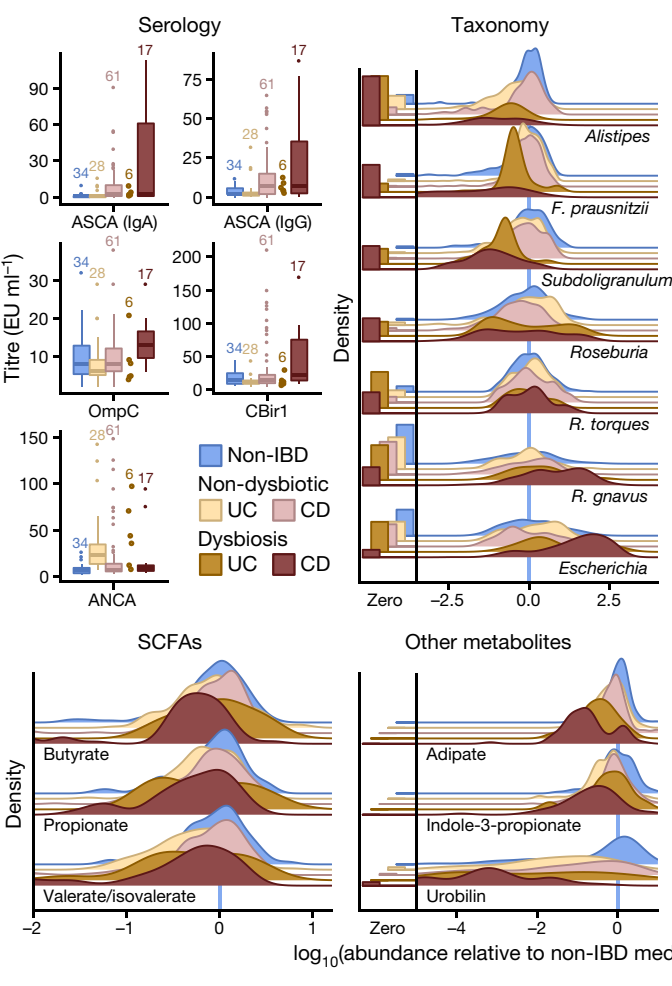

Other metabolites

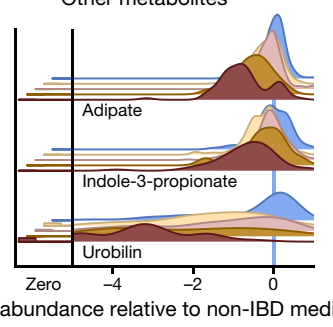

Transcription
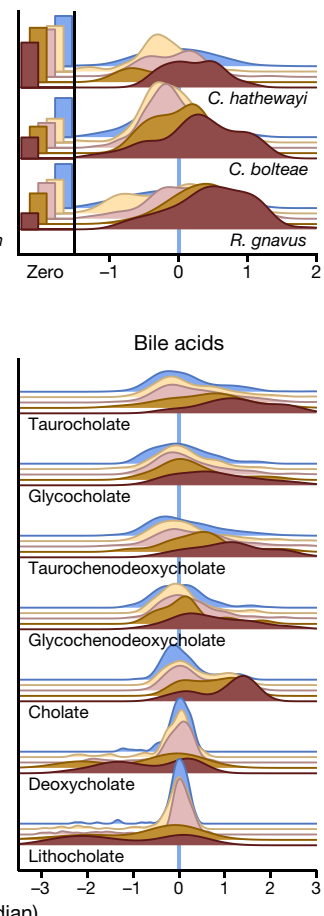

Fig. 2 Metagenomic, metatranscriptomic, and stool metabolomic profiles are disrupted during IBD activity. a, Relative abundance distributions for ten of the most cross-sectionally significantly differentially abundant metabolites in samples from individuals with IBD, as a ratio to the median relative abundance in individuals without IBD (Wald test; all FDR $P<0.003$; see Methods; Supplementary Tables 1-14). Left, fraction of samples below detection limit (see Methods). $n=546$ samples from 106 subjects. b, Relationships between two measures of disease activity: patient-reported (Harvey-Bradshaw index (HBI) in CD, $n=680$ samples from 65 subjects; simple clinical colitis activity index (SCCAI) in UC, $n=429$ samples from 38 subjects) and host molecular (faecal calprotectin (cal) ${ }^{43}, n=652$ samples from 98 subjects). Linear regression shown with $95 \%$ confidence bound. c, d, Distribution of microbial dysbiosis scores as a measure of disease activity (c, median Bray-Curtis dissimilarity between a sample and non-IBD samples;

functions in the gut ${ }^{15}$. Notably, nicotinuric acid, a metabolite of nicotinate ${ }^{16}$, was found almost exclusively in the stool of patients with IBD. Faecal calprotectin and the Harvey-Bradshaw Index (HBI), two measures of disease severity in $\mathrm{CD}$, showed no significant correlation, whereas the Simple Clinical Colitis Activity Index ${ }^{17}$ (SCCAI) in UC did correlate weakly with faecal calprotectin levels (Fig. 2b).

Notably, no metagenomic species were significantly different between samples from individuals with IBD and those from control individuals after correction for multiple hypothesis testing (Supplementary Table 1), in contrast with previous work ${ }^{4,5,18}$. We hypothesized this was due to the differentiation of study participants into two subsets, one with relatively inactive IBD (due to remission or recent onset) and the other with greater activity. This differentiation has been observed in several cohorts of patients with IBD $^{5,18}$, but it is more pronounced here because we did not take samples specifically from subjects selected for active disease. We therefore classified samples with taxonomic compositions highly unlike those of non-IBD control samples as 'dysbiotic' (Fig. 2c, Extended Data Fig. 3a-e, see Methods). Dysbiotic excursions in this cohort did not correspond with disease location (for example, ileal CD; F-test $P=0.11$, see Methods), and occurred longitudinally within subjects; they were weakly correlated with patient-reported and molecular measures of disease activity (Fig. 2d, Extended Data Fig. 3a). In total, 272 dysbiotic samples were taken during 78 full periods of dysbiosis and 9 censored periods (that is, subjects who were dysbiotic at see Methods) and its relationship with calprotectin (d, $n=652$ samples from 98 subjects). Linear regression with $95 \%$ confidence. e, KaplanMeier curves for the distributions of the durations of (left) and intervals between (right) dysbiotic episodes in UC and CD. Both are approximately exponential (fits in dashed lines), with means of 4.1 and 17.2 weeks, respectively, for UC, and 7.8 and 12.8 weeks for CD (see Methods).

f, Relative abundance distributions of significantly different metagenomic species ( $n=1,595$ samples from 130 subjects), metabolites $(n=546$ samples from 106 subjects), and microbial transcribers ( $n=818$ samples from 106 subjects) in dysbiotic samples compared to non-dysbiotic samples from the same disease group (Wald test; all FDR $P<0.05$; full results in Supplementary Tables 15-28). Also shown are antibody titres for ANCA, ASCA (IgG or IgA), anti-OmpC, and anti-CBir1 antibodies ( $n=146$ samples from 61 subjects). Boxplots show median and lower/ upper quartiles; whiskers show inner fences; sample sizes above boxes.

the end of the time series, see Methods), or $17.1 \%$ of all samples ( $n=178$ $(24.3 \%)$ in CD and $n=51$ (11.6\%) in UC). Plots of the durations of and times between dysbiotic periods were approximately exponential, suggesting that transitions are triggered, at least in part, by events with constant probability over time (and are thus potentially stochastic; Fig. 2e).

Using the resulting definition of dysbiosis, dysbiotic periods corresponded to a larger fraction of variation in all measurement types than did overall IBD phenotype (Fig. 1f, Supplementary Tables 15-28); this is likely to reflect a clearer delineation between active and less active disease states within extremely heterogeneous subjects over time. Though it is unclear which aspects of dysbiosis are causes or consequences of IBD, characterization of these changes will lead to greater understanding of microbial dynamics in disease. As in previous cross-sectional studies of established disease ${ }^{4}$, differences between dysbiotic and non-dysbiotic samples from individuals with CD were more pronounced than in those from individuals with UC (Fig. 1f). Notably, dysbiosis also distinguished between independent host measures, such as individuals with high and low ASCA (anti-Saccharomyces cerevisiae antibodies), ANCA (anti-neutrophil cytoplasm antibodies), OmpC (outer membrane protein C), and CBir1 (anti-flagellin) antibody titres in serological profiles (Fig. 2f; Fisher's combined probability test $P=0.00044$ from Wilcoxon tests between dysbiotic and non-dysbiotic CD). Dysbiosis was not significantly associated 
with demographics or medication (logistic regression with subject as random effect, all FDR $P>0.05$ ). Dysbiosis recapitulated a known decrease in alpha diversity in active disease, but we also identified numerous communities with normal complexity as dysbiotic (Extended Data Fig. 4a). Notably, taxonomic perturbations during dysbiosis mirrored those previously observed cross-sectionally in $\mathrm{IBD}^{6}$, such as the depletion of obligate anaerobes including Faecalibacterium prausnitzii and Roseburia hominis in CD and the enrichment of facultative anaerobes such as E. coli (Fig. 2f, Extended Data Fig. 4b). Ruminococcus torques and Ruminococcus gnavus, two prominent species in $\mathrm{IBD}^{19}$, were also differentially abundant in dysbiotic $\mathrm{CD}$ and $\mathrm{UC}$, respectively (FDR $P=0.041$ and 0.0087 . A smaller subset of species also increased significantly in transcriptional activity (mean total transcript relative abundance relative to genomic abundance; see Methods) as well as showing differences in abundance, including Clostridium hathewayi, Clostridium bolteae, and R. gnavus (Fig. 2f). All had significantly increased expression during dysbiosis (all FDR $P<0.07$ ), and thus their roles in IBD may be more pronounced than suggested solely by their differences in genomic abundance.

In the metabolome, SCFAs were generally reduced in dysbiosis (Fig. 2f). The reduction in butyrate in particular is consistent with the previously observed depletion of butyrate producers ${ }^{6}$ such as F. prausnitzii and R. hominis, which was also observed here (Fig. 2f). We also detected enrichment of the primary bile acid cholate and its glycine and taurine conjugates (glycocholate $q=5.2 \times 10^{-5}$, taurocholate $q=1.3 \times 10^{-5}$ ) in dysbiotic samples from participants with $\mathrm{CD}$, when compared with non-dysbiotic samples. Similarly, glycochenodeoxycholate $\left(q=1.1 \times 10^{-4}\right)$ was also enriched. By contrast, the secondary bile acids lithocholate and deoxycholate $\left(q=5 \times 10^{-7}\right.$ and $q=1.8 \times 10^{-4}$, respectively) were reduced in dysbiosis, suggesting that secondary bile-acid producing bacteria are depleted in IBD-related dysbiosis, or that transit time through the colon is too short for these compounds to be metabolized ${ }^{20,21}$. These significant metabolomic differences during microbial dysbiosis, which were concordant with changes expected during disease, provide further evidence that the dysbiosis measure is specifically relevant in IBD.

We also observed several previously undescribed biochemical differences during dysbiosis, such as large changes in acylcarnitine levels. Many acylcarnitines were significantly enriched in dysbiosis (all FDR $P<0.05$; see Extended Data Fig. 4c), whereas levels of base metabolites were typically reduced (Fig. 2f, Extended Data Fig. 4d). Of note, however, arachidonoyl carnitine (C20:4 carnitine) was reduced, and free arachidonate, a precursor of prostaglandins involved in inflammation, was increased (Fig. 2a). Like bile acids, carnitines are microbially modified compounds that can have competing phenotypic effects depending on the precise modifications: L-carnitine, for example, tends to be anti-inflammatory, whereas fatty acid-conjugated carnitine does not act uniformly on gut inflammation ${ }^{22}$. These opposing changes in biochemically related metabolites further suggest that the differences seen during dysbiosis do not stem simply from the wholesale dilution of stool. Numerous other metabolites were also significantly altered in individuals with dysbiotic IBD (117 of 548 tested known metabolites with FDR $P<0.05$; Extended Data Fig. 4d, Supplementary Table 16), showing large-scale dysregulation of metabolite pools in tandem with host- and microbiome-specific taxonomic and molecular features (Fig. 2f). Finally, although we found only a single, poorly characterized bacteriophage to be differentially prevalent in both IBD and dysbiosis (notably with reduced prevalence in IBD; Supplementary Tables 3, 17), we note that several participants showed a spike in viral load before a dysbiotic period (Supplementary Fig. 2).

\section{Decreased gut microbiome stability in IBD}

Our dense time series for stool-derived multi-omics from many subjects enabled us to carry out in-depth longitudinal analysis, integrating multiple measurements of the microbiome. Each subject's microbiome tended to diverge more from the baseline over time for metagenomic, metatranscriptomic, and metabolomic profiles (Fig. 3a; F-test power law fit $P<10^{-24}$; see Methods). These changes were most pronounced for the taxonomic profiles of individuals with $\mathrm{CD}$ and UC ( $F$-test difference in power law fits $P<10^{-9}$ ), where a the microbiome of an individual may have almost no species in common with itself at an earlier time point (dissimilarity of 1; Fig. 3a), consistent with previous observations ${ }^{9}$. Transcripts summarized within species (Extended Data Fig. 5a) showed similar trends (all $F$-test $P<8 \times 10^{-4}$ ) to metagenomic species abundances. Meanwhile, gene family transcripts (Kyoto Encyclopedia of Genes and Genomes (KEGG) Orthologues (KOs)), metabolites (Fig. 3a), and proteins (KOs, Extended Data Fig. 5a) varied much more rapidly, with essentially as much change after around two weeks as over longer time periods (increasing trends less or not significant: non-IBD, UC and CD F-test $P=0.0006,0.001$, and 0.04 , respectively for transcripts; $0.02,0.06$, and 0.003 for metabolites; and $0.5,0.15$, and 0.06 for proteomics). This indicates that these features vary rapidly in the guts of individuals with and without IBD and lack additional, more extreme excursions during disease.

We further characterized large-scale temporal differences by searching for 'shifts' in the microbiome between consecutive time points, defined as Bray-Curtis dissimilarities more similar to those between different people than within one person (Fig. 3a, Extended Data Fig. 5b, see Methods). First, considering only metagenomic taxonomic profiles, we found 166 such shifts, with 39 in individuals without IBD (of 382 total possible), 44 in individuals with UC (of 381), and 83 in individuals with CD (of 650) (Supplementary Table 29). Owing to differences in total observation times, the rate of shifts was only marginally higher in individuals with CD or UC than in non-IBD participants (2.09 and 1.83 shifts per year, respectively, compared with 1.79), and these were generally confined to a subpopulation of dysbiotic individuals (Fig. 3a). However, the species with the greatest changes in relative abundance differed markedly (Fig. 3b). Shifts in individuals without IBD occurred primarily in individuals with high abundances of Prevotella copri, which underwent repeated expansion and relaxation cycles over the course of weeks to months (Fig. 3c). This organism is of particular interest owing to its behaviour as a population-scale outgroup and its enrichment during new-onset rheumatoid arthritis ${ }^{23}$. The lack of shifts due to $P$. copri in participants with IBD was not due to an absence of $P$. copri in these individuals or an overabundance in those without IBD (6 of 27 non-IBD subjects had at least one time point with more than $10 \%$ P. copri, consistent with healthy populations ${ }^{10,24}$ ). Instead, the relative abundances that were present remained more stable in the population with IBD (Fig. 3c). Taxonomic shifts in participants with IBD mirrored earlier observations of relative reductions in obligate anaerobes and overgrowth of facultative anaerobes (Fig. 3b, Extended Data Fig. 5c), and frequently corresponded with entry into and exit from dysbiosis (28 and 23 shifts marked entries and exits in IBD, respectively, accounting for $40 \%$ of shifts in IBD). E. coli in particular contributed to a large number of shifts in IBD, although there was no clear pattern in which species it traded abundance with (Extended Data Fig. 5c, d).

When we define shifts in a similar manner for metabolomics profiles (Extended Data Fig. 5e), the rate of shifts is approximately half that seen for the metagenome (1.05 shifts per year in participants without IBD, 0.99 shifts per year in UC and 1.36 shifts per year in CD), although these data were strongly affected by the availability of fewer metabolomics samples (Extended Data Fig. 5e). We examined differences in metabolite profiles between adjacent samples from the same subjects and found significant separation by diagnosis (Fig. 3d; PERMANOVA $\left.P<10^{-4}\right)$. These differences were largely driven by unknown compounds, emphasizing the need for further compound annotation efforts and follow up to determine the significance of these compounds in IBD. Features with the greatest differences included urobilin (which showed larger differences in individuals without IBD), urate (largely in patients with CD), and a feature with an $\mathrm{m} / z$ of 152.0354 and retention time (RT) of $4.16 \mathrm{~min}$ (potentially the formic acid adduct of pyridinaldehyde), which accounted for differences largely specific to UC. The primary contributors to shifts were largely unidentified compounds (Extended Data Fig. 5f, Supplementary Table 30). HILp_QI22918, 

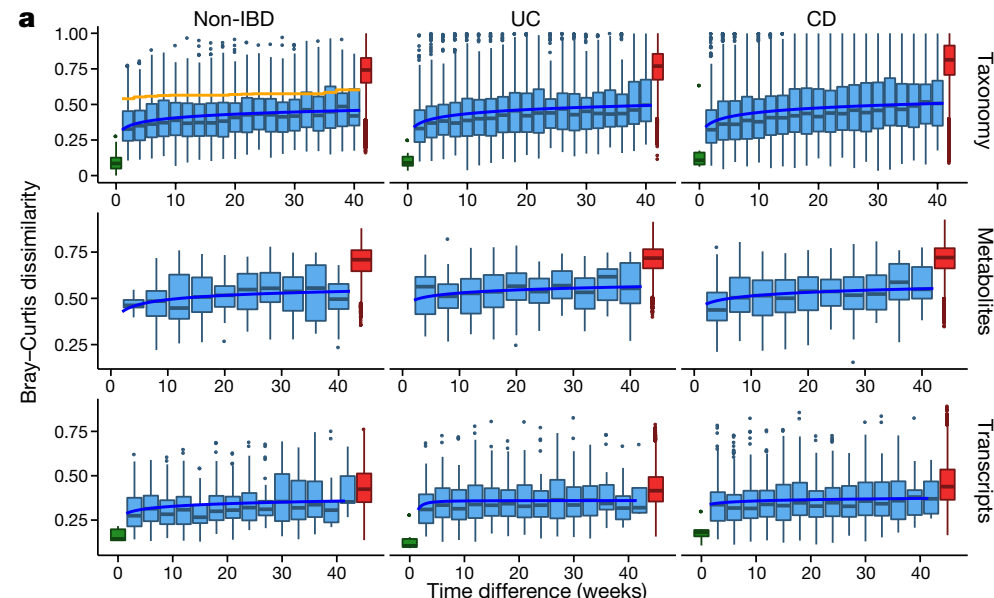

Technical replicates

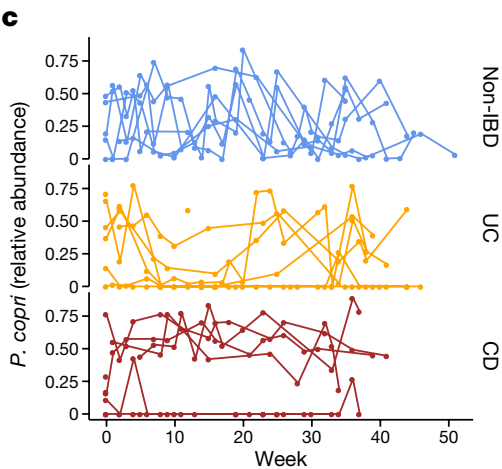

Fig. 3 Temporal shifts in the microbiome are more frequent and more extreme in IBD. a, Bray-Curtis dissimilarities within subjects as a function of intervening time difference, as compared to different people or technical replicates; calculated for metagenomic taxonomic profiles (species; $n=1,595$ samples from 130 subjects), metabolomics ( $n=546$ samples from 106 subjects), and functional profiles $\left(\mathrm{KO}^{30}\right.$ gene families; $n=818$ samples from 106 subjects). Boxplots show median and lower/ upper quartiles; whiskers show inner fences. Blue, least-squares power-law fits; orange, thresholds for microbiome shifts (see Methods). Proteomics and species-level transcripts in Extended Data Fig. 5a. Within-subject changes are significantly more extreme in $\mathrm{UC}$ and $\mathrm{CD}$ than in non-IBD for taxonomic profiles ( $F$-test $P=3.9 \times 10^{-10}$ and $1.2 \times 10^{-18}$, respectively) and transcripts $\left(P=0.00016\right.$ and $\left.1.7 \times 10^{-5}\right)$, with mixed differences for metabolites $(P=0.012$ and 0.23$)$. Technical replicates shown (when possible) at 0 weeks. $\mathbf{b}$, Shift frequencies for the top 10 species with greatest change during shifts, ranked by number of shifts as primary contributor,

an unknown feature with $m / z$ of 648.43067 and RT of $5.03 \mathrm{~min}$, contributed the most (ten) shifts exclusively in individuals with IBD. Among known compounds, methylimidazole acetic acid and urate were the primary contributors to the most shifts (four shifts each; Fig. 3e).

\section{Microbiome-associated host factors}

When we incorporated host molecular measurements, primarily from intestinal biopsies taken colonoscopically at baseline, into our analysis of the microbiome in IBD, the main influences on population variability were strikingly different from those that affected the microbiota alone. In particular, tissue location was a major driver of intestinal epithelial gene expression (Extended Data Fig. 2c) even in the face of microbial variation ${ }^{25}$ (Extended Data Fig. 2d). We therefore performed microbiome and phenotypic association analyses independently for each standardized biopsy location (see Methods).

We identified genes that were significantly differentially expressed (DEGs) in patient biopsies taken in inflamed locations of the ileum (from individuals with $\mathrm{CD}$ ) and rectum (both CD and UC) compared to individuals without IBD (Extended Data Fig. 6a). This analysis identified 305 and 920 genes, genes that were differentially expressed b
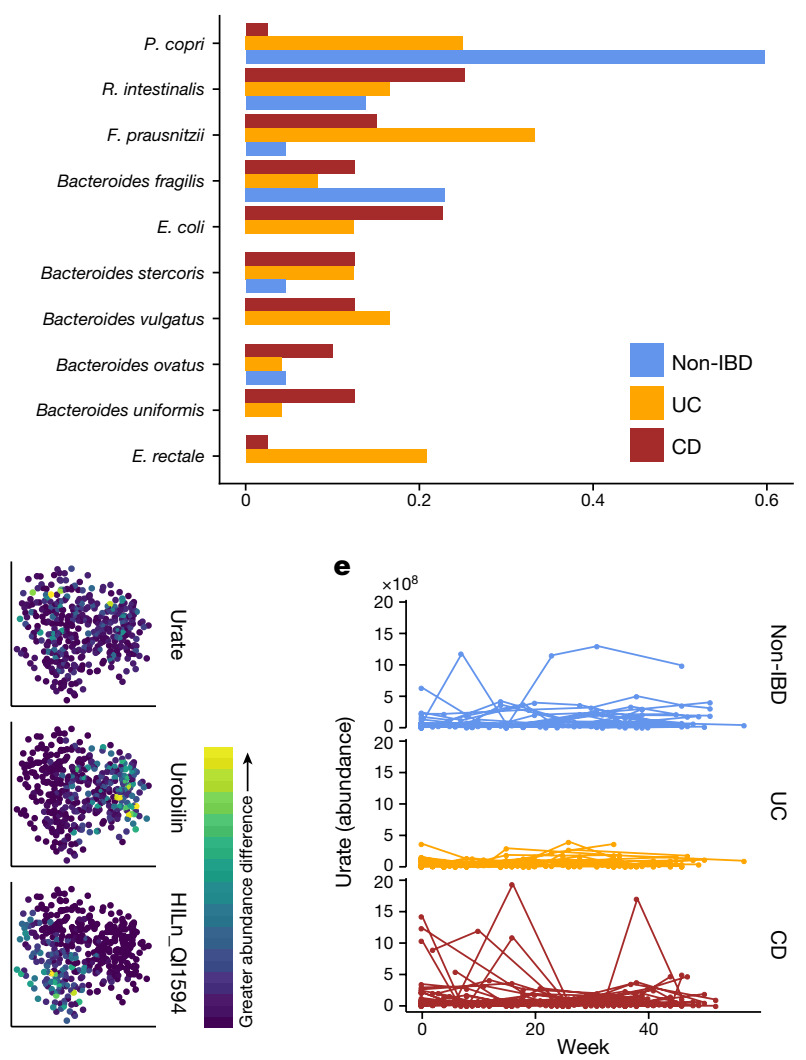

stratified by disease phenotype(s) (full table Supplementary Table 29). c, $P$. copri is of interest in arthritis ${ }^{23}$ and international populations ${ }^{44}$, and it alone retained stable abundances in $\mathrm{CD}$ but bloom-relaxation dynamics in controls (two-tailed Wilcoxon test of absolute differences between consecutive time points $P=4.2 \times 10^{-6}$ between non-IBD and UC, and $1.1 \times 10^{-4}$ between non-IBD and CD). Plot shows 22 subjects with at least one time point with more than $10 \%$ differential abundance $(n=267$ samples). d, Ordination of temporally adjacent samples within individual, based on metabolomics (Bray-Curtis principal coordinates on normalized absolute abundance differences). Disease groups separate significantly ( $n=440$ sample pairs from 106 subjects; PERMANOVA $R^{2}=2.8 \%$, $\left.P<10^{-4}\right)$. Urobilin, urate, and an unidentified untargeted feature that segregates with disease groups in the PCoA are shown (right); HILn_QI1594 (HILIC-neg method $m / z=152.0354$, RT $=4.16 \mathrm{~min}$ ). e, As in c, but for urate (two-tailed Wilcoxon test $P=0.0012$ non-IBD-UC, $P=0.044$ non-IBD-CD; $n=546$ samples from 106 subjects).

(primarily overexpressed) in the ileum and rectum, respectively, for further analysis (together representing 1,008 unique genes, negative binomial model FDR $P<0.05$ and fold-change $>1.5$; Fig. $4 \mathrm{a}$, Supplementary Table 31). These included genes that can affect commensal microorganisms directly, such as the antimicrobial CXCL6 (a cell membrane disruptor ${ }^{26}$ ) and SAA2 (which inhibits growth of Gram-negative bacteria ${ }^{27}$ ), as well as indirect microbial modulators such as DUOX2 (which produces reactive oxygen species ${ }^{28}$ ) and LCN2 (which induces microbial iron starvation through sequestration $^{29}$; Fig. 4b). Enrichment analysis testing for overrepresentation of $\mathrm{KEGG}^{30}$ pathways among DEGs also confirmed strong representation of immune-related pathways (one-sided hypergeometric test, FDR $P<0.05$ ). In particular, the IL-17 signalling pathway, components of which have been previously identified in gene expression studies of ileal biopsies from patients with $\mathrm{CD}^{31,32}$, was enriched in upregulated DEGs in both ileum and rectum (FDR $P=2.8 \times 10^{-14}$; Fig. $4 \mathrm{a}$, Supplementary Table 32). Among upregulated DEGs in rectal biopsies from patients with UC, we found further enrichment of the complement cascade (FDR $P=4.4 \times 10^{-10}$ ), a component of innate immunity ${ }^{33}$ that has been implicated in $\mathrm{IBD}^{25,34,35}$. 


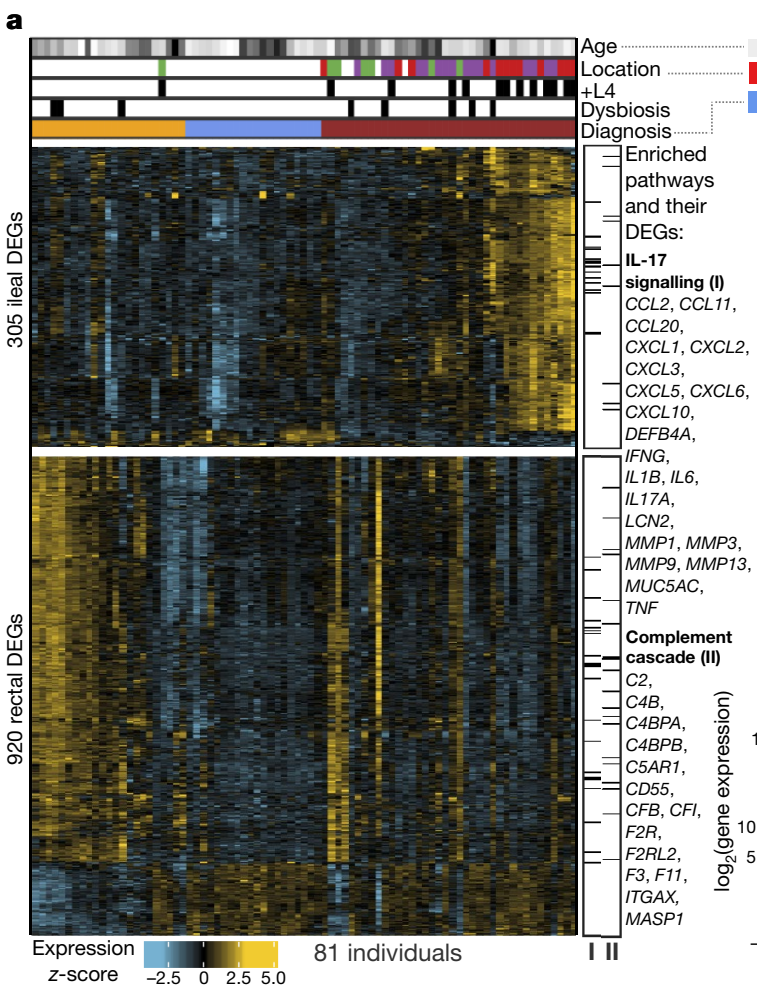

Fig. 4 | Colonic epithelial molecular processes perturbed during IBD and in tandem with multi-omic host-microbe interactions. a, Human DEGs (negative binomial FDR $P<0.05$, minimum fold change 1.5; Supplementary Table 31) from 81 subjects with paired ileal and rectal biopsies. Ordering by diagnosis, clustering within diagnosis. IL-17 signalling (I) showed strongest enrichment in ileal DEGs (FDR $\left.P=8.2 \times 10^{-12}\right)^{31}$, while the complement cascade (II) ${ }^{45}$ was enriched in rectal DEGs from patients with UC (FDR $P=5.2 \times 10^{-8}$; $\mathrm{KEGG}^{30}$ gene sets, Supplementary Table 32). Example DEGs shown with I and II b, Expression of four genes involved in host-microbe interactions ${ }^{26-29}$. Inflamed biopsy samples are shown for CD from ileum (left, $n=20$, 23, 39 independent samples for non-IBD, UC, CD respectively); for CD and $\mathrm{UC}$ in rectum (right column; $n=22,25,41$ independent samples for non-IBD, UC, CD); non-IBD samples were non-inflamed. Asterisks indicate significant differential expression compared to non-IBD

(Fisher's exact test, FDR $P<0.05 ; P$ values in Supplementary Table 31 ).

To identify the components of the microbiome that were most associated with these changes, we tested for transcripts that covaried with the relative abundance of microorganisms measured directly from the same specimens using $16 \mathrm{~S}$ amplicon sequencing. We identified 31 and 106 significant gene-operational taxonomic unit (OTU) pairs in the ileum and rectum, respectively, with no overlap between the two sites, consistent with the different overall gene expression patterns that separate them (partial Spearman correlation FDR $P<0.05$; see Methods, Extended Data Fig. 6b, Supplementary Table 33). The genes involved included known IBD-associated host-microbial interaction factors, including DUOX2 and its maturation factor DUOXA2 $2^{31,36}$, both of which were negatively associated with the abundance of Ruminococcaceae UCG 005 (OTU 89) in the ileum. The expression of several chemokine genes, some of which have reported antimicrobial properties $^{37}$ (CXCL6, CCL20), were negatively correlated with the relative abundance of Eubacterium rectale (OTU 120) in the ileum, and Streptococcus (OTU 37) and Eikenella (OTU 39) in the rectum, suggesting that these species are the most susceptible to the activity of these chemokines. Finally, although this cohort was not designed for genetic association discovery (Supplementary Discussion, Extended Data Fig. 6c, d, Supplementary Table 34), we also provide exome sequencing for 92 subjects, which may be integrated with larger populations in the future.

\section{Dynamic, multi-omic microbiome interactions}

We next searched for host and microbial molecular interactions that might underlie disease activity in IBD by constructing a large-scale cross-measurement type association network that incorporated ten microbiome measurements: metagenomic species, species-level transcription ratios, functional profiles captured as Enzyme Commission (EC) gene families (MGX, MTX and MPX), metabolites, host transcription (rectum and ileum separately), serology, and faecal calprotectin. To identify co-variation between components of the microbiome above and beyond those linked strictly to inflammation and disease state, each measurement type was first residualized using the same mixed-effects model (or linear model when appropriate) used to determine differential abundance ('adjusted' network; see Methods). This residualization uses longitudinal measurements to minimize any inter-individual variation (including IBD status), as well as dysbiotic excursions as drivers of the detected associations, and thus highlights within-person associations over time. The resulting network contained 53,161 total significant edges (FDR $P<0.05)$ and 2,916 nodes spanning features from all measurement types (Supplementary Table 35). We constructed a filtered subnetwork for visualization from the top 300 edges (by $P$ value) per measurement type in which at least one connected node was dysbiosis-associated (Fig. 4c). 
Representatives from the five stool-derived measurements occurred as hubs (defined as nodes with at least 20 connections) in this network, all of which were identified as differentially abundant in dysbiosis. Particularly connected taxonomic features (from metagenomes and metatranscriptomes) included the abundances of $F$. prausnitzii and unclassified clades related to Subdoligranulum ${ }^{38}$, which are closely phylogenetically related, although the only molecular features common to both organisms covaried with the abundances of cholesterol and inosine (Extended Data Fig. 7a). F. prausnitzii accounted for some of the strongest associations overall, including the expression of numerous ECs that were downregulated in dysbiosis. On the other hand, E. coli (and to lesser extent Haemophilus parainfluenzae) accounted for a large fraction of upregulated ECs. Members of the Roseburia genus were also associated, metatranscriptionally as well as metagenomically, with bile acids and number of acylcarnitines, suggesting that Roseburia (together with Subdoligranulum) are involved in the carnitine and bile acid dysregulation observed in IBD.

Acylcarnitines and bile acids as overall chemical classes featured prominently in the network, related in part to their changes during dysbiosis. Acylcarnitines were associated with numerous dysbiosisassociated species including $R$. hominis (nine acylcarnitines, FDR $P<0.05$; Supplementary Table 35), Klebsiella pneumoniae (three), and $H$. parainfluenzae (three), as well as expression of C. bolteae (three), suggesting that multiple scales of regulation, including long-term growth-based and short-term transcriptional, are involved. Particularly notable biochemical hubs in the network included C8 carnitine, another acylcarnitine that was significantly increased in dysbiotic $C D$, cholate, chenodeoxycholate, and taurochenodeoxycholate, which together accounted for 107 edges (6\%; Fig. 4c). Other prominent metabolite associations included several long-chain lipid hubs and the SCFA propionate; antibodies against $\mathrm{OmpC}$ were strongly associated with these, as well as with the metagenomic abundances of the numerous ECs involved in the system's biosynthesis or as interactors. Calprotectin, as the sole feature in its own measurement type, was weakly associated with a number of metabolites that were not differentially abundant in dysbiosis, as well as with the metagenomic abundance of several dysbiosisassociated ECs. Three host genes appeared in this high-significance subnetwork: ileal expression of GIP, NXPE4, and ANXA10. Expression of RNA polymerase was also a prominent node in the network, though not a hub, that was upregulated in dysbiosis (Extended Data Fig. 8). The regulation of this essential enzyme class is growth-rate-dependent ${ }^{39}$, suggesting that microbial communities as a whole are more often in higher growth conditions in dysbiotic IBD.

Finally, we also identified associations among features in the microbiome that took dysbiosis into account, resulting in a second network using the same methodology but without adjusting for dysbiosis ('unadjusted'; Supplementary Discussion, Extended Data Figs. 7b, 9, Supplementary Table 36). Together, these networks contextualize the multiple types of microbiome disruption that are observed in IBD, with associations among many molecular feature types that represent potential targets for follow-up studies on the mechanisms that underlie IBD and gastrointestinal inflammation.

\section{Conclusions}

As part of the HMP2, we have developed the IBDMDB, one of the first integrated studies of multiple molecular features of the gut microbiome that have been implicated in IBD dynamics. While overall population structure was comparable among measurements of the microbiomemetagenomic, metatranscriptomic, metabolomic, and others-each measurement identified complementary molecular components of longitudinal dysbioses in CD and UC. Some, such as taxonomic shifts in favour of aerotolerant, pro-inflammatory clades, have been captured by previous studies; others, such as greater gene expression by clostridia during disease, were discovered by the use of new measurements (metatranscriptomes). The temporal stability of multiple microbiome measurements likewise differed across IBD phenotypes and disease activity, with distinct effects on molecular components of the microbiome (including unexpected stability of the relative abundance of $P$. copri in individuals with IBD). Our data provide a catalogue of new relationships between multi-omic features identified as potentially central during IBD, in addition to data, protocols, and relevant bioinformatic approaches to enable future research.

By leveraging a multi-omic view on the microbiome, our results single out a number of host and microbial features for follow-up characterization. An unclassified Subdoligranulum species, recently shown to form a complex of new species-level clades ${ }^{38}$, was both markedly reduced in IBD and central to the functional network, associating with a wide range of IBD-linked metabolites both identifiable (for example, bile acids and polyunsaturated fatty acids) and unidentifiable. The clade is likely to contain at least seven species that are closely related to the Subdoligranulum, Gemmiger, and Faecalibacterium genera, typically butyrate producers that are considered to be beneficial, particularly in $\mathrm{IBD}^{40}$. Therefore, the isolation and characterization of additional species-especially in tandem with these associated metabolites-is likely to reveal these clades' physiological and immunological interactions and the consequences of their depletion in IBD. More generally, strain-level profiling of implicated microorganisms remains to be carried out, particularly in direct association with host epithelium and corresponding molecular changes. This profiling is feasible with existing data from this study, and will serve to pinpoint the specific organisms responsible for IBD-associated accumulation of primary unconjugated bile acids and depletion of secondary bile acids ${ }^{41}$. Only very few, low-abundance species are currently known to be capable of secondary bile acid metabolism ${ }^{42}$, and expanding the range of strains known to carry appropriate metabolic cassettes will indicate potential new targets for therapeutic restoration. Beyond short-chain fatty acids and bile acids, the large-scale acylcarnitine dysbiosis observed here may also provide a promising new target for IBD, particularly after determining whether this shift in metabolite pools is host- or microbiome-driven.

We stress that it has not yet been determined whether these multiomic features of the microbiome can predict disease events before their occurrence and that the disease-relevant time scales of distinct molecular events have not been identified (for example, static host genetics, relatively slow epigenetics or microbial growth, rapid host and microbial transcriptional changes). It may also be fruitful to seek out the earliest departures from a subject-specific baseline state that, while themselves still 'eubiotic', may predict the subsequent onset of dysbiosis or disease symptoms. Some such characterization may be possible in data from this study, although other causal analysis may be better carried out at finer-grained time scales or using interventional study designs. It will be most important to take these molecular results back to the clinic, in the form of better predictive biomarkers of IBD progression and outcome, and as a set of new host-microbe interaction targets for which treatments to ameliorate the disease may be developed.

\section{Online content}

Any methods, additional references, Nature Research reporting summaries, source data, statements of data availability and associated accession codes are available at https://doi.org/10.1038/s41586-019-1237-9.

Received: 6 February 2018; Accepted: 16 April 2019; Published online 29 May 2019.

1. Kaplan, G. G. The global burden of IBD: from 2015 to 2025. Nat. Rev. Gastroenterol. Hepatol. 12, 720-727 (2015).

2. Hugot, J. P. et al. Association of NOD2 leucine-rich repeat variants with susceptibility to Crohn's disease. Nature 411, 599-603 (2001)

3. Huang, $H$. et al. Fine-mapping inflammatory bowel disease loci to singlevariant resolution. Nature 547, 173-178 (2017).

4. Morgan, X. C. et al. Dysfunction of the intestinal microbiome in inflammatory bowel disease and treatment. Genome Biol. 13, R79 (2012)

5. Gevers, D. et al. The treatment-naive microbiome in new-onset Crohn's disease. Cell Host Microbe 15, 382-392 (2014).

6. Kostic, A. D., Xavier, R. J. \& Gevers, D. The microbiome in inflammatory bowel disease: current status and the future ahead. Gastroenterology 146, 1489-1499 (2014). 
7. Ananthakrishnan, A. N. Environmental risk factors for inflammatory bowel diseases: a review. Dig. Dis. Sci. 60, 290-298 (2015).

8. Knights, D. et al. Complex host genetics influence the microbiome in inflammatory bowel disease. Genome Med. 6, 107 (2014)

9. Halfvarson, J. et al. Dynamics of the human gut microbiome in inflammatory bowel disease. Nat. Microbiol. 2, 17004 (2017).

10. Lloyd-Price, J. et al. Strains, functions and dynamics in the expanded Human Microbiome Project. Nature 550, 61-66 (2017).

11. Vandeputte, D. et al. Stool consistency is strongly associated with gut microbiota richness and composition, enterotypes and bacterial growth rates. Gut 65, 57-62 (2016).

12. Weisshof, R. \& Chermesh, I. Micronutrient deficiencies in inflammatory bowel disease. Curr. Opin. Clin. Nutr. Metab. Care 18, 576-581 (2015).

13. Kuroki, F. et al. Multiple vitamin status in Crohn's disease. Correlation with disease activity. Dig. Dis. Sci. 38, 1614-1618 (1993)

14. Depeint, F., Bruce, W. R., Shangari, N., Mehta, R. \& O'Brien, P. J. Mitochondrial function and toxicity: role of the $B$ vitamin family on mitochondrial energy metabolism. Chem. Biol. Interact. 163, 94-112 (2006).

15. Li, J. et al. Niacin ameliorates ulcerative colitis via prostaglandin $\mathrm{D}_{2}$-mediated D prostanoid receptor 1 activation. EMBO Mol. Med. 9, 571-588 (2017).

16. Figge, H. L. et al. Comparison of excretion of nicotinuric acid after ingestion of two controlled release nicotinic acid preparations in man. J. Clin. Pharmacol. 28, 1136-1140 (1988)

17. Walmsley, R. S., Ayres, R. C., Pounder, R. E. \& Allan, R. N. A simple clinical colitis activity index. Gut 43, 29-32 (1998).

18. Frank, D. N. et al. Molecular-phylogenetic characterization of microbial community imbalances in human inflammatory bowel diseases. Proc. Natl Acad. Sci. USA 104, 13780-13785 (2007).

19. Hall, A. B. et al. A novel Ruminococcus gnavus clade enriched in inflammatory bowel disease patients. Genome Med. 9, 103 (2017).

20. Kruis, W., Kalek, H. D. Stellaard, F. \& Paumgartner, G. Altered fecal bile acid pattern in patients with inflammatory bowel disease. Digestion 35, 189-198 (1986).

21. Duboc, H. et al. Connecting dysbiosis, bile-acid dysmetabolism and gut inflammation in inflammatory bowel diseases. Gut 62, 531-539 (2013).

22. Meadows, J. A. \& Wargo, M. J. Carnitine in bacterial physiology and metabolism. Microbiology 161, 1161-1174 (2015).

23. Scher, J. U. et al. Expansion of intestinal Prevotella copri correlates with enhanced susceptibility to arthritis. eLife 2, e01202 (2013).

24. Abu-Ali, G. S. et al. Metatranscriptome of human faecal microbial communities in a cohort of adult men. Nat. Microbiol. 3, 356-366 (2018).

25. Morgan, X. C. et al. Associations between host gene expression, the mucosal microbiome, and clinical outcome in the pelvic pouch of patients with inflammatory bowel disease. Genome Biol. 16, 67 (2015).

26. Linge, H. M. et al. The human CXC chemokine granulocyte chemotactic protein 2 (GCP-2)/CXCL6 possesses membrane-disrupting properties and is antibacterial. Antimicrob. Agents Chemother. 52, 2599-2607 (2008).

27. Eckhardt, E. R. et al. Intestinal epithelial serum amyloid A modulates bacterial growth in vitro and pro-inflammatory responses in mouse experimental colitis. BMC Gastroenterol. 10, 133 (2010).

28. El Hassani, R. A. et al. Dual oxidase 2 is expressed all along the digestive tract. Am. J. Physiol. Gastrointest. Liver Physiol. 288, G933-G942 (2005).

29. Bachman, M. A., Miller, V. L. \& Weiser, J. N. Mucosal lipocalin 2 has proinflammatory and iron-sequestering effects in response to bacterial enterobactin. PLOS Pathog. 5, e1000622 (2009).

30. Kanehisa, M. et al. Data, information, knowledge and principle: back to metabolism in KEGG. Nucleic Acids Res. 42, D199-D205 (2014).

31. Haberman, Y. et al. Pediatric Crohn disease patients exhibit specific ileal transcriptome and microbiome signature. J. Clin. Invest. 124, 3617-3633 (2014).

32. Kugathasan, $\mathrm{S}$. et al. Prediction of complicated disease course for children newly diagnosed with Crohn's disease: a multicentre inception cohort study. Lancet 389, 1710-1718 (2017).

33. Hajishengallis, G., Reis, E. S., Mastellos, D. C., Ricklin, D. \& Lambris, J. D. Novel mechanisms and functions of complement. Nat. Immunol. 18, 1288-1298 (2017).

34. Ross, I. N., Thompson, R. A., Montgomery, R. D. \& Asquith, P. Significance of serum complement levels in patients with gastrointestinal disease. J. Clin. Pathol. 32, 798-801 (1979).

35. Jain, U., Otley, A. R., Van Limbergen, J. \& Stadnyk, A. W. The complement system in inflammatory bowel disease. Inflamm. Bowel Dis. 20, 1628-1637 (2014)

36. Lipinski, S. et al. DUOX2-derived reactive oxygen species are effectors of NOD2-mediated antibacterial responses. J. Cell Sci. 122, 3522-3530 (2009).

37. Yung, S. C. \& Murphy, P. M. Antimicrobial chemokines. Front. Immunol. 3, 276 (2012).

38. Pasolli, E. et al. Extensive unexplored human microbiome diversity revealed by over 150,000 genomes from metagenomes spanning age, geography, and lifestyle. Cell 176, 649-662e620 (2019).

39. Ralling, G., Bodrug, S. \& Linn, T. Growth rate-dependent regulation of RNA polymerase synthesis in Escherichia coli. Mol. Gen. Genet. 201, 379-386 (1985).

40. Sokol, H. et al. Faecalibacterium prausnitzii is an anti-inflammatory commensal bacterium identified by gut microbiota analysis of Crohn disease patients. Proc. Natl Acad. Sci. USA 105, 16731-16736 (2008).

41. Vítek, L. Bile acid malabsorption in inflammatory bowel disease. Inflamm. Bowel Dis. 21, 476-483 (2015).
42. Dawson, P. A. \& Karpen, S. J. Intestinal transport and metabolism of bile acids. J. Lipid Res. 56, 1085-1099 (2015).

43. Walsham, N. E. \& Sherwood, R. A. Fecal calprotectin in inflammatory bowel disease. Clin. Exp. Gastroenterol. 9, 21-29 (2016).

44. Truong, D. T., Tett, A., Pasolli, E., Huttenhower, C. \& Segata, N. Microbial strain-level population structure and genetic diversity from metagenomes. Genome Res. 27, 626-638 (2017). https://doi.org/10.1101/gr.216242.116.

45. Ricklin, D., Reis, E. S. \& Lambris, J. D. Complement in disease: a defence system turning offensive. Nat. Rev. Nephrol. 12, 383-401 (2016).

Acknowledgements We thank G. Ackermann, E. X. Li, J. Livny, D. McDonald, C. Moran, A. Robbins-Pianka, and S. Sun for their contributions during this project; the Broad Institute of MIT and Harvard Microbial 'Omics Core, Genomics Platform, and the Harvard T. H. Chan School of Public Health Microbiome Analysis Core for data generation and management; and T. Reimels for editorial assistance. This work was supported by National Institutes of Health grants P01DK046763 (D.P.B.M.), U01DK062413 (D.P.B.M.), U54DK102557 (D.P.B.M.), UL1TR001881 (J.B.), P30DK043351 (R.J.X.), R24DK110499 (C.H.), R01HG005969 (C.H.), U54DE023798 (C.H. and R.J.X.), National Science Foundation grant DBI-1053486 (C.H.), and Army Research Office grant W911NF-11-1-0473 (C.H.). D.S. was supported by the Swedish Research Council International Career Fellowship (4.1-2016-00416). A.B.H. is a Merck Fellow of the Helen Hay Whitney Foundation. A.N.A. was supported by the Crohn's and Colitis Foundation. D.P.B.M. was supported by the Leona M. and Harry B. Helmsley Charitable Trust. Pacific Northwest National Laboratory is a multi-program laboratory operated by Battelle for the US Department of Energy under contract DE-AC05-76RL01830.

Reviewer information Nature thanks Jeroen Raes, Philippe Schmitt-Kopplin, Eran Segal and the other anonymous reviewer(s) for their contribution to the peer review of this work.

Author contributions Study design: H.C., J.S., J. Braun, J.K.J., D.P.B.M., C.B.C., R.J.X., and C.H. Clinical coordination: A.N.A., E.A., H.C., K.L., M.P., J.S., J.L.K., H.K., C.J.M., B.W.S., W.T., R.G.W., V.Y., J. Braun, L.A.D., S.K., D.P.B.M., and H.S.W. Project managers: J.A.-P., T.W.P., E.A., H.C., T.G.G., E.A.F., and H.V. Data generation: J.L.-P., M.S., J.A.-P., T.W.P., E.A., N.J.A., D.C., T.G.G., C.J.L., M.P., J.S., R.A.W., K.B., A.D., C.D., J.L.K., C.J.M., K.A.P., W.T., J.J.t.H., R.G.W., J. Braun, D.P.B.M., J.F.P., T.S.S., C.B.C., and H.V. Bioinformatics: J.L.-P., C.A., M.S., J.A.-P., N.J.A., K.S.B., C.J.B., A.G., H.M., D.R.P., G.R., D.S., Y.V.-B., R.A.W., J. Bishai, L.J.M., R.S., A.S.-M., G.W., J. Braun, J.F.P., and C.H. Manuscript: J.L.-P., C.A., M.S., J.A.-P., K.S.B., C.J.B., A.B.H., H.M., D.R.P., G.R., D.S., L.N., C.B.C., E.A.F., H.V., and C.H. Principal Investigators: J. Braun, L.A.D., J.K.J., R.K., S.K., D.P.B.M., J.F.P., H.S.W., C.B.C., H.V., R.J.X., and C.H.

Competing interests $\mathrm{J}$. Braun is on the Scientific Advisory Board for Janssen Research \& Development, LLC. C.H. is on the Scientific Advisory Board for Seres Therapeutics. J.F.P. and N.J.A. own shares at Diversigen Inc. R.J.X. is a consultant to Novartis and Nestle.

\section{Additional information}

Supplementary information is available for this paper at https://doi.org/ 10.1038/s41586-019-1237-9.

Reprints and permissions information is available at http://www.nature.com/ reprints.

Correspondence and requests for materials regarding clinical aspects including data generation and interpretation should be addressed to R.J.X. (xavier@molbio.mgh.harvard.edu).Correspondence regarding data generation, interpretation, and analysis should be addressed to C.H.

Publisher's note: Springer Nature remains neutral with regard to jurisdictional claims in published maps and institutional affiliations.

Open Access This article is licensed under a Creative Commons Attribution 4.0 International License, which permits use, sharing, adaptation, distribution and reproduction in any medium or format, as long as you give appropriate credit to the original author(s) and the source, provide a link to the Creative Commons license, and indicate if changes were made. The images or other third party material in this article are included in the article's Creative Commons license, unless indicated otherwise in a credit line to the material. If material is not included in the article's Creative Commons license and your intended use is not permitted by statutory regulation or exceeds the permitted use, you will need to obtain permission directly from the copyright holder. To view a copy of this license, visit http://creativecommons. org/licenses/by/4.0/.

(c) The Author(s) 2019

\section{IBDMDB Investigators}

Jason Bishai ${ }^{1}$, Kevin Bullock ${ }^{4}$, Amy Deik ${ }^{4}$, Courtney Dennis ${ }^{4}$, Jess L. Kaplann ${ }^{21}$, Hamed Khalilili, Lauren J. Mclver ${ }^{2}$, Christopher J. Moran ${ }^{21}$, Long Nguyen ${ }^{3}$, Kerry A. Pierce ${ }^{4}$, Randall Schwager ${ }^{2}$, Alexandra Sirota-Madi ${ }^{1}$,

Betsy W. Stevens ${ }^{3}$, William Tan ${ }^{3}$, Johanna J. ten Hoeve ${ }^{9}$, George Weingart ${ }^{2}$, Robin G. Wilson ${ }^{3} \&$ Vijay Yajnik ${ }^{3}$ 


\section{METHODS}

Recruitment and specimen collection. Recruitment. Five medical centres participated in the IBDMDB: Cincinnati Children's Hospital, Emory University Hospital, Massachusetts General Hospital, Massachusetts General Hospital for Children, and Cedars-Sinai Medical Center. Patients were approached for potential recruitment upon presentation for routine age-related colorectal cancer screening, work up of other gastrointestinal (GI) symptoms, or suspected IBD, either with positive imaging (for example, colonic wall thickening or ileal inflammation) or symptoms of chronic diarrhoea or rectal bleeding. Participants could not have had a prior screening or diagnostic colonoscopy. Potential participants were excluded if they were unable to or did not consent to provide tissue, blood, or stool, were pregnant, had a known bleeding disorder or an acute gastrointestinal infection, were actively being treated for a malignancy with chemotherapy, were diagnosed with indeterminate colitis, or had undergone a prior, major gastrointestinal surgery such as an ileal/colonic diversion or j-pouch. Upon enrolment, an initial colonoscopy was performed to determine study strata. Subjects not diagnosed with IBD based on endoscopic and histopathologic findings were classified as 'non-IBD' controls, including the aforementioned healthy individuals presenting for routine screening, and those with more benign or non-specific symptoms. This creates a control group that, while not completely 'healthy', differs from the IBD cohorts specifically by clinical IBD status. Differences observed between these groups are therefore more likely to constitute differences specific to IBD, and not differences attributable to general GI distress. In total, 132 subjects took part in the study (Extended Data Table 1).

Regulatory compliance. The study was reviewed by the Institutional Review Boards at each sampling site: overall Partners Data Coordination (IRB \#2013P002215); MGH Adult cohort (IRB \#2004P001067); MGH Paediatrics (IRB \#2014P001115); Emory (IRB \#IRB00071468); Cincinnati Children's Hospital Medical Center (20137586); and Cedars-Sinai Medical Center (3358/CR00011696). All study participants gave written informed consent before providing samples. Each IRB has a federal wide assurance and follows the regulations established at 45 CFR Part 46. The study was conducted in accordance with the ethical principles expressed in the Declaration of Helsinki and the requirements of applicable federal regulations. Specimen collection and storage. Specimens for research (biopsies, blood draws, and stool samples) were collected during the screening colonoscopy, at up to five quarterly follow-up visits at the clinic (termed 'baseline', visit 2 , and so on, occurring at months $0,3,6,9$, and 12 ), and every two weeks by mail.

Biopsies. Biopsies were primarily gathered during the initial screening colonoscopy, where approximately four to fourteen biopsies were collected for each subject. For each location sampled (at least ileum and $10 \mathrm{~cm}$ from rectum, plus discretionary sites of inflammation), one biopsy was collected for standard histopathology at the sampling institution, two biopsies were collected and stored in RNAlater for molecular data generation (host and microbial, stored at $-20^{\circ} \mathrm{C}$ ), and one biopsy was collected and placed in a sterile tube with $5 \%$ glycerol (stored at $-80^{\circ} \mathrm{C}$ ). If possible, additional biopsies from inflamed tissue and nearby non-inflamed tissue were taken from participants with CD or UC. For adults, a second set of biopsies was also collected from each location (rectum and ileum) for epithelial cell culture (for detailed protocols see http://ibdmdb.org/protocols). All biopsies were stored for up to two months at the collection site, and shipped overnight on dry ice to Washington University for epithelial cell culture or to the Broad Institute for molecular profiling.

Blood samples. Blood samples (whole blood and serum) were taken at the quarterly clinical visits. For whole blood, $1 \mathrm{ml}$ of blood was collected and stored at $-80^{\circ} \mathrm{C}$. For serum, blood was drawn into a 5-ml SST tube, and left at room temperature for $40 \mathrm{~min}$. This was centrifuged for $15 \mathrm{~min}$ at 3,000 r.p.m. and 0.5 -ml portions were immediately aliquoted into $2-\mathrm{ml}$ microtubes. Tubes were stored at $-80^{\circ} \mathrm{C}$. Stool samples. Stool specimens were collected both at the clinical visits and every two weeks by mail using a home collection kit developed for the project (http:// ibdmdb.org/protocols) and previously validated ${ }^{46}$. Participants first deposited stool into a collection bowl suspended over a commode. They then collected two aliquots using a scoop to transfer stool into two Sarstedt 80.623 tubes: one with approximately $5 \mathrm{ml}$ molecular biology grade $100 \%$ ethanol, and one with no preservative. Stool samples were then sent from each participant by FedEx to the Broad Institute where they were processed immediately before storage at $-80^{\circ} \mathrm{C}$. The ethanol tube was centrifuged to pellet stool, which was subaliquotted, and the supernatant was transferred to a new tube for metabolomic analysis. Stool from ethanol was aliquoted into 2 - $\mathrm{ml}$ cryovials in $~ 100-200-\mathrm{mg}$ aliquots, prioritizing specimens for metaomic sequencing, metabolomics, and viromics in that order. Any remaining stool was stored in additional aliquot tubes. One hundred milligrams of the non-ethanol stool was stored for assaying faecal calprotectin and the remainder was saved in a second tube. All samples were stored at $-80^{\circ} \mathrm{C}$ after receipt before processing. This home-collection method was shown previously to produce reproducible results compared to flash-frozen samples ${ }^{46}$, consistent with previous observations across data types ${ }^{47-49}$. Note that an accurate estimate of the stool water content could not be obtained, as samples were collected by subjects and preserved in ethanol at room temperature until aliquots were generated for the different data generation platforms.

Participant and sample metadata. Descriptions of each participant and specimen were captured at baseline and accompanying each specimen collection, respectively. At baseline (that is, during or before the screening colonoscopy), subjects completed a Reported Symptoms Questionnaire, the Short Inflammatory Bowel Disease Questionnaire ${ }^{50}$, a Food Frequency Questionnaire, and an Environmental Questionnaire, and the Simple Endoscopic Score ${ }^{51}$ for CD subjects or Baron's Score ${ }^{52}$ for UC subjects was assessed.

During both follow-up visits and paired with mailed stool samples, subjects completed an Activity Index and Dietary Recall Questionnaire to assess their disease activity index (HBI for CD or SCCAI for UC) and provide a retrospective recall of their recent diet. All questionnaires, as well as detailed protocols (including product numbers), can be found on the IBDMDB data portal at http:// ibdmdb.org/protocols. Responses and metadata are available at http://ibdmdb. org/results, and summaries of phenotypes for samples and subjects are provided (Supplementary Fig. 3) along with summaries of the final time series for each subject (Supplementary Fig. 2).

Stool specimen processing. Sample selection. Sample selection proceeded in two phases, with an initial round of data generation producing a pilot metagenomics and metatranscriptomics data set, which was analysed separately ${ }^{53}$. This pilot sample selection included at least one sample per participant that was enrolled in the study at that time, two long time courses per disease group (CD, UC, non-IBD), and multiple shorter time courses, resulting in 300 samples. For a subset of 78 samples, metatranscriptomic data were generated. Samples were chosen on the basis of sample mass, preferentially selecting samples that could be re-sequenced if needed during the later data generation.

For the second, larger phase of data generation, stool samples were selected for different assays with the goal of generating data covering as many aspects of the cohort as possible, including per-subject time courses, cross-subject global time points, and samples from all patients, phenotypes, age ranges, clinical centres, and so forth (Fig. 1b). The subset of measurements performed for each sample was determined in large part by aliquot requirements (in particular, mass requirements for the assay relative to how much the patient provided) and cost.

For proteomics and metabolomics, six global time points were equally distributed over the year-long time series for as many subjects as possible. Restrictions such as available sample mass and missing samples were incorporated by selecting the nearest suitable sample in time, resulting in slight irregularities in the sampling pattern. In total, 546 metabolite profiles and 450 proteomics profiles were generated. From among these samples, 768 were selected for metagenomics, metatranscriptomics, and viromics, corresponding to 8 plates of 96 samples each. Samples already selected for proteomics or metabolomics were prioritised to facilitate integrated data analysis (316 samples had sufficient mass), resulting in six global time points for all subjects. In cases where the respective sample was not available for a subject, the nearest suitable sample in time was selected. Subjects with greater fluctuations in their HBI or SCCAI scores were then prioritized for denser sampling, resulting in 12 long time courses for 5 participants with CD, 4 with UC, and 3 without IBD. The selection also included 23 technical replicates for metagenomics, metatranscriptomics and viromics.

Finally, 576 additional samples were selected specifically for metagenomic sequencing (6 plates) resulting in a total of 1,344 metagenomic samples. Samples at previously selected global time points and long time courses that had been restricted by available mass for other measurement types were prioritized. An additional four global time points were added by this process, as well as 15 long time courses (representing 10 participants with CD, 10 with UC, and 7 without IBD), and 22 samples that had been previously sequenced for the pilot data and represented additional technical replicates. Lastly, 522 samples were selected for faecal calprotectin measurements, prioritizing samples that were selected for any other multi-omics data generation and representing a broad overview of the cohort. Of a total of 2,653 collected stool samples, 1,785 generated at least one measurement type (Fig. 1b).

Sample selection for RNA-seq and 16S sequencing from biopsies, and host genotyping from blood draws, aimed to cover the 95 subjects who contributed at least 14 stool samples, as permitted by the availability of biopsies and blood draws for each assay. Sample selection from biopsies additionally aimed to cover biopsies from inflamed and non-inflamed sites. In total, 254 biopsies were selected for RNA-seq, covering 43 participants with CD, 25 with UC, and 22 without IBD, and distributed across biopsy sites and inflammation statuses (Extended Data Fig. 6A); and 161 biopsies were selected for $16 \mathrm{~S}$ sequencing, covering 36 participants with $\mathrm{CD}, 21$ with UC, and 22 without IBD. Exome sequencing was performed for 46 participants with CD, 24 with UC, and 22 without IBD.

Sample selection for remaining sample types (RRBS, blood serology) included all samples with a suitable sample available. 
Sequencing assays. DNA and RNA isolation for metagenomics and metatranscriptomics. Total nucleic acid was extracted from one aliquot of each assayed stool sample via the Chemagic MSM I with the Chemagic DNA Blood Kit-96 from Perkin Elmer. This kit combines chemical and mechanical lysis with magnetic bead-based purification. Prior to extraction on the MSM-I, TE buffer, lysozyme, proteinase K, and RLT buffer with beta-mercaptoethanol were added to each stool sample. The stool lysate solution was vortexed to mix.

Samples were then placed on the MSM I unit to automate the following steps: M-PVA magnetic beads were added to the stool lysate solution and vortexed to mix. The bead-bound total nucleic acid was then removed from solution using a 96-rod magnetic head and washed in three ethanol-based wash buffers. The beads were then washed in a final water wash buffer. Finally, the beads were dipped in elution buffer to resuspend the DNA sample in solution. The beads were then removed from solution, leaving purified total nucleic acid eluate. The eluate was then split into two equal volumes: one for DNA and the other for RNA. SUPERase-IN solution was added to the DNA samples, and the reaction was cleaned up using AMPure XP SPRI beads. DNase was added to the RNA samples, and the reaction was cleaned up using AMPure XP SPRI beads.

DNA samples were quantified using a fluorescence-based PicoGreen assay. RNA samples were quantified using a fluorescence-based RiboGreen assay. RNA quality was assessed via smear analysis on the Caliper LabChip GX.

Metagenome sequencing. Metagenomes were generated from the resulting DNA for 1,638 stool samples, selected to obtain both a broad overview of targeted, aligned time points for all subjects (Fig. 1b), complemented by a dense sampling of subjects which tended to have greater disease activity, as determined by their HBI or SCCAI scores.

Whole-genome fragment libraries were prepared as follows. Metagenomic DNA samples were quantified by Quant-iT PicoGreen dsDNA Assay (Life Technologies) and normalized to a concentration of $50 \mathrm{pg} / \mathrm{ul}$. Illumina sequencing libraries were prepared from 100-250 pg DNA using the Nextera XT DNA Library Preparation kit (Illumina) according to the manufacturer's recommended protocol, with reaction volumes scaled accordingly. Prior to sequencing, libraries were pooled by collecting equal volumes ( $200 \mathrm{nl}$ ) of each library from batches of 96 samples. Insert sizes and concentrations for each pooled library were determined using an Agilent Bioanalyzer DNA 1000 kit (Agilent Technologies). Libraries were sequenced on HiSeq2000 or $25002 \times 101$ to yield $\sim 10$ million paired end reads. Post-sequencing de-multiplexing and generation of BAM and FASTQ files were generated using the Picard suite (https://broadinstitute.github.io/picard).

Metatranscriptome sequencing. Metatranscriptomes were generated for 855 stool samples, subsampled from metagenomic selections as above. Illumina cDNA libraries were generated using a modified version of the RNAtag-seq protocol ${ }^{54}$. In brief, $500 \mathrm{ng}-1 \mu \mathrm{g}$ of total RNA was fragmented, depleted of genomic DNA, dephosphorylated, and ligated to DNA adapters carrying $5^{\prime}$-AN8-3' barcodes of known sequence with a $5^{\prime}$ phosphate and a $3^{\prime}$ blocking group. Barcoded RNAs were pooled and depleted of rRNA using the RiboZero rRNA depletion kit (Epicentre). Pools of barcoded RNAs were converted to Illumina cDNA libraries in two main steps: (i) reverse transcription of the RNA using a primer designed to the constant region of the barcoded adaptor with addition of an adaptor to the $3^{\prime}$ end of the cDNA by template switching using SMARTScribe (Clontech) as described ${ }^{55}$; (ii) PCR amplification using primers whose $5^{\prime}$ ends target the constant regions of the $3^{\prime}$ or $5^{\prime}$ adaptors and whose $3^{\prime}$ ends contain the full Illumina P5 or P7 sequences. cDNA libraries were sequenced on the Illumina HiSeq2500 platform to generate $\sim 13$ million paired end reads.

Viromics. We selected 703 stool samples for viral profiling, following the sample selection used for metatranscriptomics and adjusted slightly only when aliquots were unavailable (Fig. 1c). Viral nucleic acids were extracted using the MagMax Viral RNA Isolation Kit (AM1939, Thermo Fisher Scientific). Viral RNA was reverse transcribed using SuperScript II RT (18064014, Thermo Fisher) and random hexamers. After short molecule and random hexamer removal using ChargeSwitch (CS12000, Thermo Fisher), molecules were amplified and tagged with a BC12-V8A2 construct ${ }^{56}$ using AccuPrimeTM Taq polymerase and cleaned with ChargeSwitch kit.

The resulting viral amplicons were normalized, pooled, and made into an Illumina library without shearing. The library (150-600 bp) was loaded into an Illumina HiSeq 2000 (Illumina, Carlsbad, CA) and sequenced using the $2 \times 100 \mathrm{bp}$ chemistry. Reads were demultiplexed into a sample bin using the barcode prefixing read-1 and read-2, allowing zero mismatches. Demultiplexed reads were further processed by trimming off barcodes, semi-random primer sequences, and Illumina adapters. This process used a custom demultiplexer and the BBDuk algorithm included in BBMap (http://sourceforge.net/projects/bbmap). The resulting trimmed data set was analysed using a pipeline created at the Alkek Center for Metagenomics and Microbiome Research at Baylor College of Medicine ${ }^{57}$. In brief, the viral analysis pipeline uses a clustering algorithm creates putative viral genomes using a mapping assembly strategy that leverages nucleotide and translated nucleotide alignment information. Viral taxonomies were assigned using a scoring system that incorporates nucleotide and translated nucleotide alignment results in a per-base fashion and optimizes for the highest resolution taxonomic rank. Metabolomics. Sample selection, receipt, and storage. Sample selection for metabolomics aimed to obtain only a broad sampling of many subjects. In total, 546 stool samples were selected for profiling (Fig. 1b). A portion of each selected stool sample (40-100 $\mathrm{mg}$ ) and the entire volume of originating ethanol preservative were stored in $15-\mathrm{ml}$ centrifuge tubes at $-80^{\circ} \mathrm{C}$ until all samples were collected. Sample processing. Samples were thawed on ice and then centrifuged $\left(4^{\circ} \mathrm{C}, 5,000 \mathrm{~g}\right)$ for $5 \mathrm{~min}$. Ethanol was evaporated using a gentle stream of nitrogen gas using a nitrogen evaporator (TurboVap LV; Biotage) and stored at $-80^{\circ} \mathrm{C}$ until all samples in the study had been dried. Aqueous homogenates were generated by sonicating each sample in $900 \mu \mathrm{l} \mathrm{H} \mathrm{H}_{2} \mathrm{O}$ using an ultrasonic probe homogenizer (Branson Sonifier 250) set to a duty cycle of $25 \%$ and output control of 2 for $3 \mathrm{~min}$. Samples were kept on ice during the homogenization process. The homogenate for each sample was aliquoted into two $10-\mu \mathrm{l}$ and two $30-\mu \mathrm{l}$ samples in 1.5 - $\mathrm{ml}$ centrifuge tubes for LC-MS sample preparation and $30 \mu \mathrm{l}$ of homogenate from each sample was transferred into a $50-\mathrm{ml}$ conical tube on ice to create a pooled reference sample. The pooled reference mixture was mixed by vortexing and then aliquoted $(100 \mu \mathrm{l}$ per aliquot) into $1.5-\mathrm{ml}$ centrifuge tubes. Aliquots and reference sample aliquots were stored at $-80^{\circ} \mathrm{C}$ until LC-MS analyses were conducted.

$L C-M S$ analyses. A combination of four LC-MS methods were used to profile metabolites in the faecal homogenates, as previously published ${ }^{58}$; two methods that measure polar metabolites, a method that measures metabolites of intermediate polarity (for example, fatty acids and bile acids), and a lipid profiling method. For the analysis queue in each method, subjects were randomized and longitudinal samples from each subject were randomized and analysed as a group. Additionally, pairs of pooled reference samples were inserted into the queue at intervals of approximately 20 samples for quality control and data standardization. Samples were prepared for each method using extraction procedures that are matched for use with the chromatography conditions. Data were acquired using LC-MS systems comprised of Nexera X2 U-HPLC systems (Shimadzu Scientific Instruments) coupled to Q Exactive/Exactive Plus orbitrap mass spectrometers (Thermo Fisher Scientific). The method details are summarized below.

LC-MS Method 1: HILIC-pos (positive ion mode MS analyses of polar metabolites). LC-MS samples were prepared from stool homogenates $(10 \mu \mathrm{l})$ by protein precipitation with the addition of nine volumes of 74.9:24.9:0.2 v/v/v acetonitrile/methanol/formic acid containing stable isotope-labelled internal standards (valine- $\mathrm{d} 8$, Isotec; and phenylalanine- $\mathrm{d} 8$, Cambridge Isotope Laboratories). The samples were centrifuged $\left(10 \mathrm{~min}, 9,000 \mathrm{~g}, 4^{\circ} \mathrm{C}\right)$, and the supernatants injected directly onto a $150 \times 2$-mm Atlantis HILIC column (Waters). The column was eluted isocratically at a flow rate of $250 \mu \mathrm{l} / \mathrm{min}$ with $5 \%$ mobile phase A $(10 \mathrm{mM}$ ammonium formate and $0.1 \%$ formic acid in water) for $1 \mathrm{~min}$ followed by a linear gradient to $40 \%$ mobile phase B (acetonitrile with $0.1 \%$ formic acid) over $10 \mathrm{~min}$. MS analyses were carried out using electrospray ionization in the positive ion mode using full scan analysis over $m / z 70-800$ at 70,000 resolution and 3-Hz data acquisition rate. Additional MS settings are: ion spray voltage, $3.5 \mathrm{kV}$; capillary temperature, $350^{\circ} \mathrm{C}$; probe heater temperature, $300^{\circ} \mathrm{C}$; sheath gas, 40 ; auxiliary gas, 15; and S-lens RF level 40.

LC-MS Method 2: HILIC-neg (negative ion mode MS analysis of polar metabolites). LC-MS samples were prepared from stool homogenates $(30 \mu \mathrm{l})$ by protein precipitation with the addition of four volumes of $80 \%$ methanol containing inosine-15N4, thymine- $\mathrm{d} 4$ and glycocholate- $\mathrm{d} 4$ internal standards (Cambridge Isotope Laboratories). The samples were centrifuged $\left(10 \mathrm{~min}, 9,000 \mathrm{~g}, 4^{\circ} \mathrm{C}\right)$ and the supernatants were injected directly onto a $150 \times 2.0-\mathrm{mm}$ Luna NH 2 column (Phenomenex). The column was eluted at a flow rate of $400 \mu \mathrm{l} / \mathrm{min}$ with initial conditions of $10 \%$ mobile phase $\mathrm{A}(20 \mathrm{mM}$ ammonium acetate and $20 \mathrm{mM}$ ammonium hydroxide in water) and $90 \%$ mobile phase $\mathrm{B}(10 \mathrm{mM}$ ammonium hydroxide in $75: 25 \mathrm{v} / \mathrm{v}$ acetonitrile/methanol) followed by a 10 -min linear gradient to $100 \%$ mobile phase A. MS analyses were carried out using electrospray ionization in the negative ion mode using full scan analysis over $m / z 60-750$ at 70,000 resolution and $3 \mathrm{~Hz}$ data acquisition rate. Additional MS settings are: ion spray voltage, $-3.0 \mathrm{kV}$; capillary temperature, $350^{\circ} \mathrm{C}$; probe heater temperature, $325^{\circ} \mathrm{C}$; sheath gas, 55; auxiliary gas, 10; and S-lens RF level 40.

LC-MS Method 3: C18-neg (negative ion mode analysis of metabolites of intermediate polarity; for example, bile acids and free fatty acids). Stool homogenates $(30 \mu \mathrm{l})$ were extracted using $90 \mu \mathrm{l}$ methanol containing PGE2-d4 as an internal standard (Cayman Chemical Co.) and centrifuged $\left(10 \mathrm{~min}, 9,000 \mathrm{~g}, 4^{\circ} \mathrm{C}\right)$. The supernatants $(10 \mu \mathrm{l})$ were injected onto a $150 \times 2.1-\mathrm{mm}$ ACQUITY BEH C18 column (Waters). The column was eluted isocratically at a flow rate of $450 \mu \mathrm{l} / \mathrm{min}$ with $20 \%$ mobile phase $\mathrm{A}(0.01 \%$ formic acid in water) for $3 \mathrm{~min}$ followed by a linear gradient to $100 \%$ mobile phase B ( $0.01 \%$ acetic acid in acetonitrile) over $12 \mathrm{~min}$. MS analyses were carried out using electrospray ionization in the negative ion mode using full scan analysis over $m / z 70-850$ at 70,000 resolution and $3 \mathrm{~Hz}$ data 
acquisition rate. Additional MS settings are: ion spray voltage, $-3.5 \mathrm{kV}$; capillary temperature, $320^{\circ} \mathrm{C}$; probe heater temperature, $300^{\circ} \mathrm{C}$; sheath gas, 45 ; auxiliary gas, 10; and S-lens RF level 60.

LC-MS Method 4: C8-pos. Lipids (polar and nonpolar) were extracted from stool homogenates $(10 \mu \mathrm{l})$ using $190 \mu \mathrm{l}$ isopropanol containing 1-dodecanoyl-2tridecanoyl-sn-glycero-3-phosphocholine as an internal standard (Avanti Polar Lipids; Alabaster, AL). After centrifugation (10 min, 9,000g, ambient temperature), supernatants $(10 \mu \mathrm{l})$ were injected directly onto a $100 \times 2.1$-mm ACQUITY BEH C8 column ( $1.7 \mu \mathrm{m}$; Waters). The column was eluted at a flow rate of $450 \mu \mathrm{l} / \mathrm{min}$ isocratically for $1 \mathrm{~min}$ at $80 \%$ mobile phase A (95:5:0.1 v/v/vl $10 \mathrm{mM}$ ammonium acetate/methanol/acetic acid), followed by a linear gradient to $80 \%$ mobile phase B (99.9:0.1 v/v methanol/acetic acid) over $2 \mathrm{~min}$, a linear gradient to $100 \%$ mobile phase B over $7 \mathrm{~min}$, and then $3 \mathrm{~min}$ at $100 \%$ mobile phase B. MS analyses were carried out using electrospray ionization in the positive ion mode using full scan analysis over $m / z 200-1,100$ at 70,000 resolution and $3 \mathrm{~Hz}$ data acquisition rate. Additional MS settings are: ion spray voltage, $3.0 \mathrm{kV}$; capillary temperature, $300^{\circ} \mathrm{C}$; probe heater temperature, $300^{\circ} \mathrm{C}$; sheath gas, 50 ; auxiliary gas, 15 ; and S-lens RF level 60 .

Metabolomics data processing. Raw LC-MS data were acquired to the data acquisition computer interfaced to each LC-MS system and then stored on a robust and redundant file storage system (Isilon Systems) accessed via the internal network at the Broad Institute. Nontargeted data were processed using Progenesis QIsoftware (v 2.0, Nonlinear Dynamics) to detect and de-isotope peaks, perform chromatographic retention time alignment, and integrate peak areas. Peaks of unknown ID were tracked by method, $\mathrm{m} / \mathrm{z}$ and retention time. Identification of nontargeted metabolite LC-MS peaks was conducted by: i) matching measured retention times and masses to mixtures of reference metabolites analysed in each batch; and ii) matching an internal database of $>600$ compounds that have been characterized using the Broad Institute methods. Temporal drift was monitored and normalized with the intensities of features measured in the pooled reference samples.

Proteomics. Sample selection and LC-MS/MS. Sample selection for proteomics largely followed sample selection for metabolomics (Fig. 1b, c), with slight adjustments when aliquots were unavailable. In total, 447 stool samples were targeted for profiling. From the selected samples, proteins were proteolytically digested using trypsin, and each digest was subjected to automated offline high-pH reversedphase fractionation with fraction concatenation. LC-MS/MS analysis for each fraction was performed using a Thermo Scientific Q-Exactive Orbitrap mass spectrometer at UCLA, outfitted with a custom-built nano-ESI interface. Samples were loaded onto an in-house packed capillary LC column $(70 \mathrm{~cm} \times 75 \mu \mathrm{m}, 3-\mu \mathrm{m}$ particle size), and data were acquired for $120 \mathrm{~min}$. Precursor MS spectra were collected over $400-2,000 \mathrm{~m} / \mathrm{z}$, followed by data-dependent MS/MS spectra of the twelve most abundant ions, using a collision energy of $30 \%$. A dynamic exclusion time of $30 \mathrm{~s}$ was used to discriminate against previously analysed ions.

Peptide identification and protein data roll-up. Mass spectra from the resulting analyses were evaluated using the $\mathrm{MSGF}^{+}$software ${ }^{59}$ v10072 using the HMP 1 gut reference genomes (HMP_Refgenome-gut_2015-06-18). In brief, after conversion of the metagenomic assemblies into predicted open reading frames (for example, predicted proteins), libraries were created using the forward and reverse direction to allow determination of FDR. The reverse decoy database allows measurement of the rate of detection of false hits, which in turn allows calculation of FDR and appropriate filtering of the data to maximize real peptide identifications while minimizing spurious ones. $\mathrm{MSGF}^{+}$was then used to search the experimental mass spectra data against both the forward and reverse decoy databases. Cut-offs for data included: $\mathrm{MSGF}^{+}$spectra probability $\left(>1 \times 10^{10}\right.$, equivalent to a BLAST $e$ value), mass accuracy ( \pm 20 p.p.m.), protein level FDR of $1 \%$ and one unique peptide per protein identification.

Faecal calprotectin. Faecal calprotectin was quantified for 652 stool samples, which were stored at $-80^{\circ} \mathrm{C}$ without preservative before processing. Sample selection focused on obtaining a broad survey of all subjects rather than detailed time series (Fig. 1b). Calprotectin was quantified using QUANTA Lite Calprotectin ELISA (Inova Diagnostics 704770) following the manufacturer's protocol. Between 80 and $120 \mathrm{mg}$ of stool was used for input. Incubation time before stopping the reaction was adjusted to obtain $\mathrm{OD}_{405}$ values in the suggested range for assay.

Biopsy specimen processing. Co-isolation of DNA and RNA from frozen tissue. DNA and RNA were extracted from RNA-later-preserved biopsies using the AllPrep DNA/RNA Universal Kit from Qiagen. Biological samples were cut into 20-25-mg pieces on a dry ice batch, then placed in tubes with a steel bead for mechanical homogenization and a highly denaturing guanidine isothiocyanatecontaining buffer, which immediately inactivates DNases and RNases to ensure isolation of intact DNA and RNA. After homogenization, the lysate was passed through an AllPrep DNA Mini spin column. This column, in combination with the high-salt buffer, allows selective and efficient binding of genomic DNA. On-column proteinase $\mathrm{K}$ digestion in optimized buffer conditions allows purification of high DNA yields from all sample types. The column was then washed and DNA was eluted in TE buffer. Flow-through from the AllPrep DNA Mini spin column was digested by proteinase $\mathrm{K}$ in the presence of ethanol. This optimized digestion, together with the subsequent addition of further ethanol, allowed for appropriate binding of total RNA, including miRNA, to the RNeasy Mini spin column. Samples were then digested with DNase I to ensure high-yields of DNA-free RNA. Contaminants were efficiently washed away and RNA was eluted in water. $16 S$ rRNA gene profiling. We selected 178 biopsies for $16 \mathrm{~S}$ amplicon-based taxonomic profiling. The $16 \mathrm{~S}$ rRNA gene-sequencing protocol was adapted from the Earth Microbiome Project ${ }^{60}$ and the Human Microbiome Project ${ }^{61-63}$. In brief, bacterial genomic DNA was extracted from the total mass of the biopsied specimens using the MoBIO PowerLyzer Tissue and Cells DNA isolation kit and sterile spatulas for tissue transfer. The $16 \mathrm{~S}$ rDNA V4 region was amplified from the extracted DNA by PCR and sequenced in the MiSeq platform (Illumina) using the $2 \times 250 \mathrm{bp}$ paired-end protocol, yielding pair-end reads that overlapped almost completely. The primers used contained adapters for MiSeq sequencing and single-index barcodes such that PCR products may be pooled and sequenced directly ${ }^{61}$, targeting at least 10,000 reads per sample.

Read pairs were demultiplexed and merged using USEARCH v7.0.109064. Sequences were clustered into OTUs at a similarity threshold of $97 \%$ using the UPARSE algorithm ${ }^{65}$. OTUs were subsequently mapped to a subset of the SILVA database ${ }^{66}$ containing only sequences from the V4 region of the $16 \mathrm{~S}$ rRNA gene to determine taxonomies. Abundances were then recovered by mapping the demultiplexed reads to the UPARSE OTUs, producing the final taxonomic profiles. The 150 samples with $\geq 1,000$ mapped reads were used in downstream analyses.

Host RNA-seq. cDNA library construction. In total, 252 biopsies were selected for transcriptional profiling. Total RNA was quantified using the Quant-iT RiboGreen RNA Assay Kit and normalized to $5 \mathrm{ng} / \mu \mathrm{l}$. Following plating, $2 \mu \mathrm{l}$ of ERCC controls (using a 1:1,000 dilution) were spiked into each sample. An aliquot of $200 \mathrm{ng}$ for each sample was transferred into library preparation, which was an automated variant of the Illumina TruSeq Stranded mRNA Sample Preparation Kit. This method preserves strand orientation of the RNA transcript. It uses oligo dT beads to select mRNA from the total RNA sample. It is followed by heat fragmentation and cDNA synthesis from the RNA template. The resultant 500-bp cDNA then goes through library preparation (end repair, base 'A' addition, adaptor ligation, and enrichment) using Broad Institute designed indexed adapters substituted in for multiplexing. After enrichment the libraries were quantified using Quant-iT PicoGreen (1:200 dilution). After normalizing samples to $5 \mathrm{ng} / \mu \mathrm{l}$, the set was pooled and quantified using the KAPA Library Quantification Kit for Illumina Sequencing Platforms. The entire process is in 96-well format and all pipetting is done by either Agilent Bravo or Hamilton Starlet.

Illumina sequencing. Pooled libraries were normalized to $2 \mathrm{nM}$ and denatured using $0.1 \mathrm{~N} \mathrm{NaOH}$ before sequencing. Flowcell cluster amplification and sequencing were performed according to the manufacturer's protocols using either the HiSeq 2000 or HiSeq 2500. Each run was a 101-bp paired-end with an eight-base index barcode read. Data were organized using the Broad Institute Picard Pipeline which includes de-multiplexing and lane aggregation.

Blood specimen processing. Serological analysis. We analysed 210 serum samples for expression of ANCA, ASCA, anti-OmpC, and anti-CBir1 by ELISA as previously described ${ }^{67,68}$. Antibody levels were determined and the results expressed as ELISA units $(\mathrm{EU} / \mathrm{ml})$, which are relative to laboratory standards consisting of pooled, antigen-reactive sera from of patients with well-characterized disease. DNA isolation from whole blood. DNA was extracted using Chemagic MSM I with the Chemagic DNA Blood Kit-96 from Perkin Elmer. The kit combines chemical and mechanical lysis with magnetic bead-based purification. Whole blood samples were incubated at $37^{\circ} \mathrm{C}$ for $5-10 \mathrm{~min}$ to thaw. The blood was then transferred to a deep well plate with protease and placed on the Chemagic MSM I. The following steps were automated on the MSM I.

M-PVA magnetic beads were added to the blood and protease solution. Lysis buffer was added to the solution and vortexed to mix. The bead-bound DNA was then removed from solution using a 96-rod magnetic head and washed in three ethanol-based wash buffers to eliminate cell debris and protein residue. The beads were then washed in a final water wash buffer. Finally, the beads were dipped in elution buffer to resuspend the DNA. The beads were then removed from solution, leaving purified DNA eluate. The resulting DNA samples were quantified using a fluorescence-based PicoGreen assay.

Host exome sequencing. Ninety-two host exomes were sequenced from DNA extracts using previously published methods ${ }^{69}$. Whole-exome libraries were constructed and sequenced on an Illumina HiSeq 4000 sequencer with 151-bp pairedend reads. Output from Illumina software was processed by the Picard pipeline to yield BAM files containing calibrated, aligned reads.

Library construction. Library construction was performed as described ${ }^{69}$ with some slight modifications. Initial genomic DNA input into shearing was reduced from $3 \mu \mathrm{g}$ to $50 \mathrm{ng}$ in $10 \mu \mathrm{l}$ solution and enzymatically sheared. In addition, for adaptor ligation, dual-indexed Illumina paired end adapters were replaced with 
palindromic forked adapters with unique 8-base index sequences embedded within the adaptor and added to each end.

In-solution hybrid selection for exome enrichment. In-solution hybrid selection was performed using the Illumina Rapid Capture Exome enrichment kit with $38 \mathrm{Mb}$ target territory ( $29 \mathrm{Mb}$ baited). The targeted region includes $98.3 \%$ of the intervals in the Refseq exome database. Dual-indexed libraries were pooled into groups of up to 96 samples before hybridization. The liquid handling was automated on a Hamilton Starlet. The enriched library pools were quantified using PicoGreen after elution from streptavadin beads and then normalized to a range compatible with sequencing template denature protocols.

Preparation of libraries for cluster amplification and sequencing. Following sample preparation, the libraries prepared using forked, indexed adapters were quantified using quantitative PCR (purchased from KAPA biosystems), normalized to $2 \mathrm{nM}$ using the Hamilton Starlet Liquid Handling system, and pooled by equal volume using the Hamilton Starlet Liquid Handling system. Pools were then denatured using $0.1 \mathrm{~N} \mathrm{NaOH}$. Denatured samples were diluted into strip tubes using the Hamilton Starlet Liquid Handling system.

Cluster amplification and sequencing. Cluster amplification of the templates was performed according to the manufacturer's protocol (Illumina) using the Illumina cBot. Flow cells were sequenced on HiSeq 4000 Sequencing-by-Synthesis Kits, then analysed using RTA2.7.3

Host genetic data processing. Host genetic exome sequence data were processed using the Broad Institute sequencing pipeline by the Data Sciences Platform (Broad Institute). This was done in three steps: pre-processing (including reads mapping, alignment to a reference genome and data cleanup), variant discovery (including per-sample variant calling and joint genotyping), and variant filtering to produce callset ready for downstream genetic analysis, using Genome Analysis Toolkit (GATK) (detailed documentation at https://software.broadinstitute.org/ gatk/documentation/).

Reduced representation bisulfite sequencing. Reduced representation bisulfite sequencing (RRBS) libraries were prepared for 221 biopsies and 228 blood samples as described previously ${ }^{70}$ with modifications detailed below. In brief, genomic DNA samples were quantified using a Quant-It dsDNA high sensitivity kit (ThermoFisher, Q33120) and normalized to a concentration of $10 \mathrm{ng} / \mu \mathrm{l}$. A total of $100 \mathrm{ng}$ of normalized genomic DNA was digested with MspI in a 20- $\mu \mathrm{l}$ reaction containing $1 \mu \mathrm{l} \mathrm{MspI} \mathrm{(20} \mathrm{U/ \mu l)} \mathrm{(NEB,} \mathrm{R0106L)} \mathrm{and} 2 \mu \mathrm{l}$ of $10 \times$ CutSmart Buffer (NEB, B7204S). MspI digestion reactions were then incubated at $37^{\circ} \mathrm{C}$ for $2 \mathrm{~h}$ followed by a $15 \mathrm{~min}$ incubation at $65^{\circ} \mathrm{C}$.

Next, A-tailing reactions were performed by adding $1 \mu \mathrm{ldNTP}$ mix (containing $10 \mathrm{mM}$ dATP, $1 \mathrm{mM}$ dCTP and $1 \mathrm{mM}$ dGTP) (NEB, N0446S), $1 \mu \mathrm{l} \mathrm{Klenow} 3^{\prime}-5^{\prime}$ exo $(\mathrm{NEB}, \mathrm{M} 0212 \mathrm{~L})$ and $1 \mu \mathrm{l} 10 \times$ CutSmart Buffer in a total reaction volume of $30 \mu \mathrm{l}$. A-tailing reactions were then incubated at $30^{\circ} \mathrm{C}$ for $20 \mathrm{~min}$, followed by $37^{\circ} \mathrm{C}$ for $20 \mathrm{~min}$, followed by $65^{\circ} \mathrm{C}$ for $15 \mathrm{~min}$.

Methylated Illumina sequencing adapters ${ }^{70}$ were then ligated to the A-tailed material ( $30 \mu \mathrm{l})$ by adding $1 \mu \mathrm{l} 10 \times$ CutSmart Buffer, $5 \mu \mathrm{l} 10 \mathrm{mM}$ ATP (NEB, P0756S), $1 \mu \mathrm{l} \mathrm{T} 4$ DNA Ligase (2,000,000 U/ml) (NEB, M0202M) and $2 \mu \mathrm{l}$ methylated adapters in a total reaction volume of $40 \mu \mathrm{l}$. Adaptor ligation reactions were then incubated at $16^{\circ} \mathrm{C}$ overnight $(16-20 \mathrm{~h})$ followed by incubation at $65^{\circ} \mathrm{C}$ for $15 \mathrm{~min}$. Adaptor ligated material was purified using $1.2 \times$ volumes of Ampure $\mathrm{XP}$ according to the manufacturer's recommended protocol (Beckman Coulter, A63881).

Following adaptor ligation, bisulfite conversion and subsequent sample purification were performed using the QIAGEN EpiTect kit according to the manufacturer's recommended protocol designated for DNA extracted from FFPE tissues (QIAGEN, 59104). Two rounds of bisulfite conversion were performed yielding a total of $40 \mu \mathrm{l}$ bisulfite-converted DNA.

In order to determine the minimum number of PCR cycles required for final library amplification, $50 \mu \mathrm{l}$ PCR reactions containing $3 \mu \mathrm{l}$ bisulfite-converted DNA, $5 \mu \mathrm{l} 10 \times$ PfuTurbo Cx hotstart DNA polymerase buffer, $0.5 \mu 1100 \mathrm{mM} \mathrm{dNTP}(25 \mathrm{mM}$ each dNTP) (Agilent, 200415), $0.5 \mu l$ Illumina TruSeq PCR primers $(25 \mu \mathrm{M}$ each primer $^{70}$ and $1 \mu \mathrm{l}$ PfuTurbo Cx hotstart DNA polymerase (Agilent, 600412) were prepared. Reactions were then split equally into four separate tubes and thermocycled using the following conditions: denature at $95^{\circ} \mathrm{C}$ for $2 \mathrm{~min}$ followed by $X$ cycles of $95^{\circ} \mathrm{C}$ for $30 \mathrm{~s}, 65^{\circ} \mathrm{C}$ for $30 \mathrm{~s}, 72^{\circ} \mathrm{C}$ for $45 \mathrm{~s}$ (where $X$ number of cycles $=11$, 13,15 and 17), followed by a final extension at $72^{\circ} \mathrm{C}$ for $7 \mathrm{~min}$. PCR products were purified using $1.2 \times$ volumes of Ampure XP and analysed on an Agilent Bioanalyzer using a High Sensitivity DNA kit (Agilent, 5067-4626). Once the optimal number of PCR cycles was determined, $200-\mu \mathrm{l}$ PCR reactions were prepared using $24 \mu \mathrm{l}$ bisulfite-converted DNA, $20 \mu \mathrm{l} 10 \times$ PfuTurbo Cx hotstart DNA polymerase buffer, $2 \mu \mathrm{l} 100 \mathrm{mM}$ dNTPs ( $25 \mathrm{mM}$ each), $2 \mu \mathrm{l}$ Illumina TruSeq PCR primers $(25 \mu \mathrm{M}$ each) and $4 \mu \mathrm{l}$ PfuTurbo Cx hotstart DNA polymerase with the thermal cycling conditions listed above. PCR reactions were purified using $1.2 \times$ volumes of Ampure XP according to the manufacturer's recommended protocol and analysed on an Agilent Bioanalyzer using a High Sensitivity DNA kit.
RRBS sequencing produced an average of $15.0 \mathrm{M}$ reads (s.d. $4.0 \mathrm{M}$ reads) over all 504 samples, with 448 (88.9\%) samples exceeding 10M reads. Samples were analysed with Picard 2.9.4 using default parameters, resulting in a mean alignment rate to the human genome hg19 of $95.1 \%$. Mean CpG coverage was $8.9 \times$ (s.d. $2.1 \%$ ). As expected, $99.9 \%$ (s.d. $0.02 \%$ ) of non-CpG bases and $49.8 \%$ (s.d. $2.8 \%$ ) of $\mathrm{CpG}$ bases were converted.

Data handling. Informatics for microbial community sequencing data. For metagenomes and metatranscriptomes, sequencing reads from each sample in a pool were demultiplexed based on their associated barcode sequence using custom scripts. Up to one mismatch in the barcode was allowed provided it did not make assignment of the read to a different barcode possible. Barcode sequences were removed from the first read as were terminal Gs from the second read that may have been added by SMARTScribe during template switching.

Taxonomic and functional profiles were generated with the bioBakery metaomics workflow ${ }^{71}$ v0.9.0 (http://huttenhower.sph.harvard.edu/biobakery_workflows). In brief, reads mapping to the human genome were first filtered out using KneadData 0.7.0. Taxonomic profiles of shotgun metagenomes were generated using MetaPhlAn2 $2^{72} \mathrm{v} 2.6 .0$, which uses a library of clade-specific markers to provide pan-microbial (bacterial, archaeal, viral, and eukaryotic) profiling (http:// huttenhower.sph.harvard.edu/metaphlan2). Functional profiling was performed by HUMAnN2 ${ }^{73}$ v0.11.0 (http://huttenhower.sph.harvard.edu/humann2). HUMAnN2 constructs a sample-specific reference database from the pangenomes of the subset of species detected in the sample by MetaPhlAn2 (pangenomes are precomputed representations of the open reading frames of a given species ${ }^{74}$ ). Sample reads are mapped against this database to quantify gene presence and abundance on a per-species basis. A translated search is then performed against a UniRef-based protein sequence catalogue ${ }^{75}$ (UniRef release 2014_07) for all reads that fail to map at the nucleotide level. The result are abundance profiles of gene families (UniRef90s), for both metagenomics and metatranscriptomics, stratified by each species contributing those genes, and which can then be summarized to higher-level gene groupings such as ECs or KOs.

Sample counts in Fig. $1 \mathrm{~b}$ represent the numbers of raw products available. To ensure a reasonable read depth in each sample, only samples (metagenomes and metatranscriptomes) with at least 1 million reads (after human filtering) and at least one non-zero microbial abundance detected by MetaPhlAn2 were used in downstream analyses (Fig. 1c and later). In total, this was 1,595 metagenomic and 818 metatranscriptomic samples. Principal coordinates plots were generated with the cmdscale function in the R package stats. Visualizations were principally generated using ggplot $2^{76}$.

Species-level metaomic functional profile summaries. Functional profiles per clade (typically species) were further quantified by summing the total sum-normalized stratified abundance attributed to each organism in the HUMAnN2 functional profiles from both metatranscriptomics and metagenomics. For metatranscriptomic analyses, the expression ratio for the species was then also defined as the ratio between these sums.

Metaproteomic gene family functional profiles. Gene family profiles were generated from metaproteomic peptides using UniRef 90 identifiers by mapping peptide sequences to the Diamond-annotated reference in HUMAnN2 v0.11.0 with v0.8.22.84 ${ }^{77}$. Each peptide was mapped to the UniRef90 with the highest per cent identity (minimum 90\% match).

Statistical methods and association testing. PERMANOVA and Mantel tests. Omnibus testing was performed on Bray-Curtis dissimilarity matrices from MGX, MTX, MPX, and biopsy measurements. Functional profiles (MGX, MTX, and MPX) were first summarized to the KO level using HUMAnN2. Profiles were first normalized before calculation of dissimilarities. Dietary distance matrices were calculated by ordering the dietary intake frequencies from less to more frequent, assigning integers to these levels, and calculating the Manhattan distance.

Quantifications of covariation between measurement types in Fig. le were done using Mantel tests. To quantify cross-sectional ('inter-individual') covariation, we first produced an average profile for each subject by taking the feature-wise mean over all samples from the subject. Subject-subject dissimilarity matrices were then generated and compared using the mantel.rtest function in the R package ape4. To quantify longitudinal covariation, we first generated the complete sample-sample dissimilarity matrix, but only calculate the Mantel test statistic (the Pearson correlation between distances) from distances between samples from the same subject. Significance in this case was assessed using a permutation test with permutations limited within-subject.

Quantifications of variance explained in Fig. If were calculated using PERMANOVA with the adonis function in the R package Vegan ${ }^{78}$. Apart from the All row in Fig. If, the total variance explained by each variable was calculated independently of other variables (that is, as the sole variable in the model) to avoid issues related to variable ordering, and should thus be considered the total variance explainable by that variable. To account for the repeated measures present for all measurement types tested, relevant permutations were performed blocked within 
subject for variables that change over time (medication, biopsy location, inflammation status, and dysbiosis). Meanwhile, variables that were constant (or change slowly enough to be considered constant) across samples from the same subject (age, sex, body mass index (BMI), race, recruitment site, diagnosis, and disease location) were first permuted across subjects and samples were relabelled with the variable from their permuted subject. To determine the significance of models including inter-individual variance (the Subject and All rows), permutations were performed freely. For subjects with incomplete records for BMI, we imputed the mean BMI of the remaining population. The All row is the total variance explained when including all other variables in the model.

Differential microbiome feature abundance. Differential abundance (DA) analysis of all microbial measurement types (except for viruses, which were modelled as presence/absence binary features) were tested as follows. First, an appropriate transformation/normalization method was applied: arcsine square-root transformation for microbial taxonomic and functional relative abundances, $\log$ transformation (with pseudo count 1 for zero values) for metabolite profiles and protein abundances, and log transform with no pseudocount for expression ratios (non-finite values removed). Transformed abundances were then fit with the following per-feature linear mixed-effects model:

\section{feature (intercept) + diagnosis + diagnosis/dysbiosis + antibiotic use + consent age $+(1 \mid$ recruitment site $)+(1 \mid$ subject $)$}

That is, in each per-feature multivariable model, recruitment sites and subjects were included as random effects to account for the correlations in the repeated measures (denoted by ( $1 \mid$ recruitment site) and ( $1 \mid$ subject), respectively) and the transformed abundance of each feature was modelled as a function of diagnosis (a categorical variable with non-IBD as the reference group) and dysbiosis state as a nested binary variable (with non-dysbiotic as reference) within each IBD phenotype (UC, CD, and non-IBD), while adjusting for consent age as a continuous covariate, and antibiotics as as binary covariate. Pearson's residual values from the above linear mixed effects models were retained for use in subsequent analyses (see 'Cross-measurement type interaction testing').

Fitting was performed with the nlme package in $\mathrm{R}^{79}$ (using the lme function), where significance of the association was assessed using Wald's test (except for viruses, where a logistic random effects model was considered with the glmer function from the lmer R package). Nominal $P$ values were adjusted for multiple hypothesis testing with a target FDR of 0.25 . In order to reduce the effect of zeroinflation in microbiome data, features with no variance or with $>90 \%$ zeros were removed before fitting linear models. In addition, a variance filtering step was applied to remove features with very low variance. To further remove the effect of redundancy in $\mathrm{KO}$ gene family abundances (explainable by at most a single taxon), only features (both DNA and RNA) with low correlation with individual microbial abundances (Spearman correlation coefficient $<0.6$ ) were retained.

Differential host gene expression. Differentially expressed human genes between disease groups were quantified using a quasi-likelihood negative binomial generalized log-linear model (glmQLFit), implemented in the edgeR package in $\mathrm{R}^{80,81}$. Analysis was performed separately for each section of the intestine on genes with at least 2 CPM (counts per million) in 10 or more samples, with significance threshold FDR $P<0.05$ and $>1.5 \log$-fold change. Gene enrichment analysis was performed on differentially expressed genes against the KEGG database ${ }^{82}$ using a one-sided hypergeometric test in the package limma ${ }^{83}$.

Associations with host gene expression. Associations between host gene expression and biopsy taxonomic profiles were assessed using partial Spearman correlation, accounting for BMI, age at consent, sex and diagnosis. Association testing was performed for each biopsy location independently, as biopsy location was shown to heavily influence expression profiles (Figs. 1f, $4 \mathrm{a}, \mathrm{b}$ ). This simpler method was used rather than the more complex procedure outlined above for microbial measurement types since host gene expression, once filtered by biopsy location, do not have the same repeat measures problem as the microbial measurement types, allowing a simpler test.

Genetic associations. Genetic principal components for IBDMDB subjects as well as 1,000 Genomes subjects ${ }^{84}$ were calculated for a set of independent SNPs overlapping between the two data sets and pruned on the basis of linkage disequilibrium (LD). Pruning was first performed in HMP2 using the -indep-pairwise 15001500.1 command in PLINK ${ }^{85}$ by calculating $\operatorname{LD}\left(r^{2}\right)$ for each pair of SNPs within a window of 1,500 SNPs, removing one of a pair of SNPs if $r^{2}>0.1$ and repeating this procedure by shifting the window 150 SNPs forward. We then used the 1,000 Genomes reference phase 3 version $5 \mathrm{a}$ data for 2,504 participants (http://bochet.gcc.biostat.washington.edu/beagle/1000_ Genomes_phase3_v5a) to merge with the HMP2 pruned data, resulting in 7,227 overlapping independent SNPs. Using these, we performed genome-wide estimation of identity-by-descent allele sharing on the combined data set using the-genome function in PLINK, followed by calculation of genetic principal components using the -cluster-mds-plot function for the first two principal components (Fig. 1a).

For association analyses, we used first 20 genetic principal components as covariates, obtained from the same identity-by-descent sharing matrix using the -cluster-pca 20 function. We targeted associations in five loci that had strong previously reported associations with IBD and/or have been implicated in microbial interactions ${ }^{86-88}$. To avoid confounding by ancestry, we restricted the analysis to subjects of European ancestry, excluding eight subjects with exomes available from other ancestral backgrounds. When available, we used reported SNPs that had minor allele frequency of at least $5 \%$ and Hardy-Weinberg equilibrium $P<5 \times 10^{-5}$. If not, we used close proxies ( $\mathrm{LD} r^{2}<0.8$ in CEU population using 1,000 genomes phase 3 version 5 reference via http://analysistools.nci.nih.gov/ LDlink (MST1, FUT2, IRGM, NKX2-3) or SNPs from the gnomAD browser at http://gnomad.broadinstitute.org (PTGER4)).

We used the following linear mixed effect model with the SNP as a predictor variable, coded with an additive genetic model with the outcome as the arcsine-square root transformed microbial relative abundance measured from stool metagenomes. Age, sex, antibiotic and immunosuppressant use, and the first 20 genetic principal components (PCs) were fitted as covariates with subjects as the random effect:

$$
\begin{gathered}
\text { taxon } \sim \text { intercept }+ \text { SNP }+ \text { antibiotic use }+ \text { sex }+ \text { age } \\
+ \text { recruitment site }+ \text { PC1 }- \text { PC20 }+(1 \mid \text { subject })
\end{gathered}
$$

Optimization was performed using the lme function (from the nlme R package), with $P$ values calculated using the Wald test.

Associations between the rs 1042712 SNP of the $L C T$ locus ${ }^{89}$ and self-reported milk intake from dietary recall forms were tested using the same mixed effect model. Reported dairy intake options were assigned the following numeric values for regression: 1 ('yesterday, 3 or more times'), 2 ('yesterday, 1 to 2 times'); 3 ('within the past 2 to 3 days'), 4 ('within the past 4 to 7 days'), and 5 ('did not consume in last 7 days').

Density ridgeline plots of differentially abundant features. To visualize the abundances of features that showed significantly different abundances by one of the tests above (Fig. 2, Extended Data Fig. 4), the bandwidth for kernel density estimation was selected independently for the portion of each feature above the detection limit (non-'zero') using the Sheather and Jones method ${ }^{90}$. Density estimates were scaled such that the maximum density for the plot spanned the distance between baselines for a given disease group. Samples below the detection limit are represented as barplots on the left, where a bar that spans the distance between baselines for a disease group represents $100 \%$ zeros. Density estimates were then additionally scaled by the fraction of non-zero samples such that relative differences in densities between groups with differing fractions of zeros are accurately represented. For both density estimates and fraction of zeros, samples were weighted by the inverse of the number of samples obtained from that subject, to avoid biasing estimates towards subjects with more densely sampled time series.

Dysbiosis analyses. Dysbiosis score. To identify samples with highly divergent (dysbiotic) metagenomic microbial compositions, as a complement to baseline disease diagnosis, we defined a dysbiosis score based on Bray-Curtis dissimilarities to non-IBD metagenomes. First, a 'reference set' of samples was constructed from non-IBD subjects by taking all samples after the 20th week after the subject's first stool sample. This was chosen because a subset of the non-IBD subjects at the start of their respective time series may not yet have overcome any gastrointestinal symptoms that triggered the initial visit to a doctor, though these were ultimately not caused by IBD. The dysbiosis score of a given sample was then defined as the median Bray-Curtis dissimilarity to this reference sample set, excluding samples that came from the same subject (Fig. 2c).

To identify samples that were highly divergent from the reference set, we thresholded the dysbiosis score at the 90th percentile of this score for non-IBD samples. This therefore identifies samples with a feature configuration that has a less than $10 \%$ probability of occurring in a participant without IBD. By this measure, 272 metagenomes were classified as dysbiotic. Samples from participants with CD or UC were overrepresented in the dysbiotic set, with $24.3 \%$ and $11.6 \%$ of their samples classified as dysbiotic, respectively. As expected, these samples also tended to locate in the extremes of the taxonomic ordination based on metagenomes (Extended Data Fig. 3b, c). Dysbiosis was unevenly distributed among subjects (Extended Data Fig. 3d), with some subjects remaining dysbiotic for all or most of their time series, while others remained non-dysbiotic for their entire time series.

To lend additional support to the definition of dysbiosis (that is, as outliers by one type of microbiome profile), we tested the concordance between dysbiosis classifications made using the same statistical definition, but applied to metabolomic rather than taxonomic profiles. That is, we defined a metabolomic dysbiosis score as the median Bray-Curtis dissimilarity of one metabolomic profile to the non-IBD metabolomic profiles (after the 20th week), and defined the dysbiosis threshold as the 90th percentile of this distribution among non-IBD metabolomic profiles. 
We then compared these dysbiosis classifications with those from the nearest metagenomic sample (up to two weeks, see 'Cross-measurement type temporal matching') and found that dysbiotic samples identified by metagenomics were 4.6 times more likely to be dysbiotic by metabolomics (Fisher's exact $P=5.9 \times 10^{-9}$ ), showing that dysbiosis measurements are highly consistent across measurement types.

To test the sensitivity of the dysbiosis classification to the choice of reference data set, we also performed the dysbiosis classification using the HMP1-II stool samples ${ }^{10}$ as the reference sample set instead of the non-IBD samples. The resulting dysbiosis scores (Extended Data Fig. 3e) were highly concordant (Spearman $\rho=0.86 ; P<2.2 \times 10^{-16}$ ), as were the dysbiosis classifications (odds ratio of 56 ; Fisher's exact $P<2.2 \times 10^{-16}$ ). This shows that, despite the inclusion of subjects with other conditions in the non-IBD group here, as well as large differences in measurement technologies between the data sets, the dysbiosis classification is highly robust. Furthermore, 43 out of 426 (10.1\%) of non-IBD samples were classified as dysbiotic using the HMP1-II samples as reference, falling remarkably close to the $10 \%$ expected by the definition and showing that the enrichment of IBD samples in the dysbiotic set is not simply a consequence of the definition. Dysbiosis durations and intervals. Samples of the dysbiosis durations and intervals were obtained by taking the difference in time between stool metagenomes in which the dysbiosis state changes, that is, the time from the first dysbiotic sample in an excursion into dysbiosis until the next non-dysbiotic sample was taken as one sample of the dysbiosis duration distribution. If the subject's time series ended before this transition occurred, this resulted in a 'censored' duration or interval (Fig. 2e). Estimates of the durations of and time between dysbioses were then obtained from a censored maximum likelihood estimator for the mean of an exponential distribution. This incorporates the censored durations and intervals into the estimate to avoid underestimating these durations owing to limited observation times.

Association of dysbiosis with disease location. We tested for a relationship between the Montreal disease location classification in $\mathrm{CD}$ and periods of dysbiosis to ensure that dysbiosis was not simply detecting different disease locations. For this, an $F$-test of no significance was used with a Kenward-Roger approximation of degrees of freedom ${ }^{91}$ in a logistic random effects regression that models dysbiosis as the binary outcome with subjects as a random effect and disease location as covariate, as implemented in the function glmer in the R package lme4. Only individuals with $\mathrm{CD}$ were considered.

Temporal analyses. Power-law fits to Bray-Curtis dissimilarities. Power-law fits to species-level metagenomic, metatranscriptomic, and metabolite Bray-Curtis dissimilarities (Fig. 3a, Extended Data Fig. 5a) were performed by fitting a power-law curve with free intercept by least-squares using the neldermead function from the $\mathrm{R}$ package nloptr. Significance was assessed using an $F$-test to compare the fit model with a flat line. Significance of the difference of the fit between disease groups was also assessed using an $F$-test, comparing a model jointly fit to both disease groups with separate fits to each group.

Microbiome shifts. A microbiome 'shift' was defined as having occurred between two consecutive time points from the same person if the Bray-Curtis dissimilarity between their profiles was more likely to have come from a comparison between samples from different people rather than from the same person (Extended Data Fig. 5b). As an individual's microbial profile naturally changes over time $e^{10,92}$, the Bray-Curtis threshold at which this occurs will increase with the time difference between samples. To determine these thresholds, kernel density estimates were generated for the distribution of Bray-Curtis dissimilarities between profiles from different individuals without IBD and between samples from the individuals without IBD at a range of time differences, using the density function in $\mathrm{R}$. The point at which the inter-individual density estimate exceeded the intra-individual density estimate was then taken as the threshold to define a 'shift', with the additional constraint that this must be a monotonically increasing function of the time difference between samples (Fig. 3a). Metabolomic shifts were defined similarly, although owing to the more sparse temporal sampling of the metabolomics data (Extended Data Fig. 1) and lack of a strong upward trend in Bray-Curtis dissimilarities with time difference (Fig. 3a), only a single threshold was used based on the distribution of Bray-Curtis dissimilarities from comparisons within-subject over time in participants without IBD. Heatmaps of shift differences were generated using the R package pheatmap $1.0 .10^{93}$.

Longitudinal multi-omic study design. Owing to the large variation in microbial profiles between people (Supplementary Fig. 2), with relatively smaller variation within subjects over time (Fig. 3a), longitudinal study designs have the potential to be higher-powered than purely cross-sectional studies, particularly in their ability to self-control individuals and to capture transitions between phenotypes (or after interventions) of interest ${ }^{94}$. Here, although some subjects remained in a dysbiotic state far longer than others (Extended Data Fig. 3d), the heterogeneity observed was enough to discover differences in measurement types other than where dysbiosis was defined. For example, subjects who had unusual, disease-associated microbiome taxonomic profiles also proved to have generally shifted serological and/or metabolomic profiles at corresponding time points. Noting these dysbiotic time points offered a complementary set of differences to what was visible cross-sectionally (Fig. 2).

Among microbially related measurements, metabolomics provided the most robust separation between disease and dysbiosis groups, possibly because it integrated a combination of host, microbial, and dietary differences (Fig. 1e, f). Thus, despite the challenges presented by untargeted metabolomics, such as unknown compound identification, the presence of redundant and background signals, and the complexity of the stool matrix, this measurement type often provides a robust characterization of subjects, their disease state, and individual small molecules that interface between host and microbiome. Conversely, the current state of viromics assays and reference databases makes this more challenging to work with, although the importance of the virome in microbial community dynamics ${ }^{95}$ will make this an extremely interesting feature space going forward.

All longitudinal microbial measurement types showed significant variation within two weeks (Fig. 3a, Extended Data Fig. 5a). This suggests that even higher sampling rates may be needed to catch relevant microbial variation, particularly before the onset of more severe clinical symptoms. Our sampling protocol also did not account for other potential sources of within-subject variation, such as transit time or the precise portion of each whole stool that was sampled. In this data set, we thus cannot distinguish between temporal and technical variation within subjects. To mitigate the higher costs associated with processing additional samples, a future study aiming to achieve higher temporal resolution might proceed in two phases, thanks to the coupling between data types (Fig. 1e): first, collect samples at a higher frequency, and process these with metagenomic or $16 \mathrm{~S}$ sequencing. Then proceed with more expensive and detailed data generation only for samples taken specifically around periods of interest, such as periods of dysbiosis identified during the first stage. To this end, the sampling rate can also be tuned to target a particular probability of missing a dysbiosis period.

Integrative analyses. Lenient cross-measurement type temporal matching. For comparison between multiple measurement types, we first constructed sets of samples corresponding to the same biosample across data sets. However, exact matches were not always possible, for example, owing to specimen limitations during sample selection (see 'Sample selection') or to samples that failed quality control. In these cases, matching sample sets across data sets were created using nearby samples. During this process, a degree of leniency was allowed in the matching, allowing samples up to a given time difference (two or four weeks) apart to be 'matched'. To perform this matching (sample numbers in Fig. 1c and Extended Data Fig. 1b), we used the following algorithm.

For a given set of measurement types to be matched for a particular subject, find the first time window in which all measurement types have at least one produced sample. Next, within this window, find the time point with the most measurement types produced; in the event of a tie, select earlier time points. Finally, for each data set, select the nearest sample to this target time point that is within the time window, breaking ties towards earlier time points. This set of selected samples comprises one 'matched' sample. For each data set, all samples up to and including the later of the selected sample or the target time point are then disregarded from future consideration (and thus any sample will be included in at most only one matched time point). This process is repeated for each subject until no such window exists.

Cross-measurement type interaction testing. Significant associations between features from multiple measurement types were identified using two different models: an 'unadjusted' model of associations that are mainly due to dysbiosis, and an 'adjusted' model that emphasizes associations in addition to those that are dysbiosislinked. Associations in both cases incorporated features from ten data sets: metagenomic species, species-level transcription ratios, functional profiles at the EC level (MGX, MTX and MPX), metabolites, host transcription (rectal and ileal separately), serology and faecal calprotectin.

To detect adjusted associations, we first obtained residuals of features from the above data types fit to a mixed-effects model including subjects as random effects as above for the differential abundance testing (or a simple linear model without the random effects when only baseline samples were used) and adjusting for age, sex, diagnosis, dysbiosis status, antibiotic and immunosuppressant use, and bowel surgery status. Residuals from subjects with fewer than four samples in their time series for the measurement type were ignored, and for measurements with no longitudinal samples (for example, serology and host transcriptomics measurements), the residualization was repeated with the first available samples using a simple linear model without random effects. This allowed the identification of significant (FDR $P<0.05$ for most measurement types, $P<0.25$ for serology) Spearman associations using HAllA 0.8.17 (hierarchical all-against-all association testing, http://huttenhower.sph.harvard.edu/halla, Supplementary Table 35). As subject-specific random effects and covariate effects were removed from these residuals, the resulting correlations are likely to be independent of all sources of 
inter-individual variation as well as confounding effects due to the covariates. See Fig. 4c and Extended Data Figs. 7-9 for summary visualizations of these results. Similarly, unadjusted associations were identified using the same procedure, but without including dysbiosis as a covariate (Supplementary Table 36). Network visualization was done using Cytoscape ${ }^{96}$ 3.6.0.

Reporting summary. Further information on research design is available in the Nature Research Reporting Summary linked to this paper.

\section{Data availability}

Protocols and data (both raw and summarized to data type-dependent profiles) are available at the IBDMDB website (https://ibdmdb.org), the HMP DACC web portal (https://www.hmpdacc.org/ihmp/), and Qiita ${ }^{97}$ (https://qiita.ucsd.edu/). Sequence data are available from SRA BioProject PRJNA398089. Expression data have been deposited in the NCBI Gene Expression Omnibus ${ }^{98}$ and is accessible through GEO Series accession number GSE111889. Metabolomics data are available at the NIH Common Fund's Metabolomics Data Repository and Coordinating Center (supported by NIH grant U01-DK097430) website, the Metabolomics Workbench (http://www.metabolomicsworkbench.org), where it has been assigned Project ID PR000639. Mass spectrometry proteomics data have been deposited to the ProteomeXchange Consortium via the PRIDE $^{99}$ partner repository with the data set identifiers PXD008675 and 10.6019/PXD008675. Reprints and permissions information is available at www.nature.com/reprints.

\section{Code availability}

Bioinformatics workflows for metagenomics and metatranscriptomics data are available at https://bitbucket.org/biobakery/hmp2_workflows. Analysis scripts are available at https://bitbucket.org/biobakery/hmp2_analysis.

46. Franzosa, E. A. et al. Relating the metatranscriptome and metagenome of the human gut. Proc. Natl Acad. Sci. USA 111, E2329-E2338 (2014).

47. Vogtmann, E. et al. Comparison of collection methods for fecal samples in microbiome studies. Am. J. Epidemiol. 185, 115-123 (2017).

48. Loftfield, E. et al. Comparison of collection methods for fecal samples for discovery metabolomics in epidemiologic studies. Cancer Epidemiol. Biomarkers Prev. 25, 1483-1490 (2016).

49. Voigt, A. Y. et al. Temporal and technical variability of human gut metagenomes. Genome Biol. 16, 73 (2015)

50. Jowett, S. L., Seal, C. J., Barton, J. R. \& Welfare, M. R. The short inflammatory bowel disease questionnaire is reliable and responsive to clinically important change in ulcerative colitis. Am. J. Gastroenterol. 96, 2921-2928 (2001).

51. Daperno, M. et al. Development and validation of a new, simplified endoscopic activity score for Crohn's disease: the SES-CD. Gastrointest. Endosc. 60, 505-512 (2004)

52. Baron, J. H., Connell, A. M. \& Lennard-Jones, J. E. Variation between observers in describing mucosal appearances in proctocolitis. BMJ 1, 89-92 (1964)

53. Schirmer, M. et al. Dynamics of metatranscription in the inflammatory bowel disease gut microbiome. Nat. Microbiol. 3, 337-346 (2018).

54. Shishkin, A. A. et al. Simultaneous generation of many RNA-seq libraries in a single reaction. Nat. Methods 12, 323-325 (2015).

55. Zhu, Y. Y., Machleder, E. M., Chenchik, A., Li, R. \& Siebert, P. D. Reverse transcriptase template switching: a SMART approach for full-length cDNA library construction. Biotechniques 30, 892-897 (2001).

56. Clem, A. L., Sims, J., Telang, S., Eaton, J. W. \& Chesney, J. Virus detection and identification using random multiplex (RT)-PCR with $3^{\prime}$-locked random primers. Virol. J. 4, 65 (2007)

57. Ajami, N. J., Wong, M. C., Ross, M. C., Lloyd, R. E. \& Petrosino, J. F. Maximal vira information recovery from sequence data using VirMAP. Nat. Commun. 9 3205 (2018)

58. Kostic, A. D. et al. The dynamics of the human infant gut microbiome in development and in progression toward type 1 diabetes. Cell Host Microbe $\mathbf{1 7}$ 260-273 (2015)

59. Kim, S. \& Pevzner, P. A. MS-GF+ makes progress towards a universal database search tool for proteomics. Nat. Commun. 5, 5277 (2014).

60. Thompson, L. R. et al. A communal catalogue reveals Earth's multiscale microbial diversity. Nature 551, 457-463 (2017).

61. Caporaso, J. G. et al. Ultra-high-throughput microbial community analysis on the Illumina HiSeq and MiSeq platforms. ISME J. 6, 1621-1624 (2012).

62. Human Microbiome Project Consortium. Structure, function and diversity of the healthy human microbiome. Nature 486, 207-214 (2012).

63. Human Microbiome Project Consortium. A framework for human microbiome research. Nature 486, 215-221 (2012).

64. Edgar, R. C. Search and clustering orders of magnitude faster than BLAST. Bioinformatics 26, 2460-2461 (2010)

65. Edgar, R. C. UPARSE: highly accurate OTU sequences from microbial amplicon reads. Nat. Methods 10, 996-998 (2013).

66. Pruesse, E. et al. SILVA: a comprehensive online resource for quality checked and aligned ribosomal RNA sequence data compatible with ARB. Nucleic Acids Res. 35, 7188-7196 (2007)

67. Landers, C. J. et al. Selected loss of tolerance evidenced by Crohn's disease-associated immune responses to auto- and microbial antigens. Gastroenterology 123, 689-699 (2002).
68. Targan, S. R. et al. Antibodies to CBirl flagellin define a unique response that is associated independently with complicated Crohn's disease. Gastroenterology 128, 2020-2028 (2005).

69. Fisher, $\mathrm{S}$. et al. A scalable, fully automated process for construction of sequence-ready human exome targeted capture libraries. Genome Biol. 12, R1 (2011).

70. Gu, H. et al. Preparation of reduced representation bisulfite sequencing libraries for genome-scale DNA methylation profiling. Nat. Protocols 6 468-481 (2011).

71. Mclver, L. J. et al. bioBakery: A meta'omic analysis environment. Bioinformatics 34, 1235-1237 (2018)

72. Truong, D. T. et al. MetaPhIAn2 for enhanced metagenomic taxonomic profiling. Nat. Methods 12, 902-903 (2015).

73. Franzosa, E. A. et al. Species-level functional profiling of metagenomes and metatranscriptomes. Nat. Methods 15, 962-968 (2018).

74. Huang, K. et al. MetaRef: a pan-genomic database for comparative and community microbial genomics. Nucleic Acids Res. 42, D617-D624 (2014).

75. Suzek, B. E., Wang, Y., Huang, H., McGarvey, P. B. \& Wu, C. H. UniRef clusters: a comprehensive and scalable alternative for improving sequence similarity searches. Bioinformatics 31, 926-932 (2015).

76. Wickham, H. ggplot2: Elegant Graphics for Data Analysis (Springer, New York, 2016).

77. Buchfink, B., Xie, C. \& Huson, D. H. Fast and sensitive protein alignment using DIAMOND. Nat. Methods 12, 59-60 (2015).

78. Oksanen, J. et al. vegan: Community Ecology Package. R package version 2.5-3. https://CRAN.R-project.org/package=vegan (2018).

79. Pinheiro, J. et al. nlme: Linear and Nonlinear Mixed Effects Models. R package version 3.1-108. https://CRAN.R-project.org/package=nlme (2013).

80. Robinson, M. D., McCarthy, D. J. \& Smyth, G. K. edgeR: a Bioconductor package for differential expression analysis of digital gene expression data. Bioinformatics 26, 139-140 (2010).

81. McCarthy, D. J., Chen, Y. \& Smyth, G. K. Differential expression analysis of multifactor RNA-seq experiments with respect to biological variation. Nucleic Acids Res. 40, 4288-4297 (2012).

82. Kanehisa, M., Furumichi, M., Tanabe, M., Sato, Y. \& Morishima, K. KEGG: new perspectives on genomes, pathways, diseases and drugs. Nucleic Acids Res. 45, D353-D361 (2017)

83. Ritchie, M. E. et al. limma powers differential expression analyses for RNA-sequencing and microarray studies. Nucleic Acids Res. 43, e47 (2015).

84. The 1000 Genomes Project Consortium A global reference for human genetic variation. Nature 526, 68-74 (2015).

85. Purcell, S. et al. PLINK: a tool set for whole-genome association and population-based linkage analyses. Am. J. Hum. Genet. 81, 559-575 (2007).

86. Jostins, L. et al. Host-microbe interactions have shaped the genetic architecture of inflammatory bowel disease. Nature 491, 119-124 (2012)

87. Liu, J. Z. et al. Association analyses identify 38 susceptibility loci for inflammatory bowel disease and highlight shared genetic risk across populations. Nat. Genet. 47, 979-986 (2015).

88. Hall, A. B., Tolonen, A. C. \& Xavier, R. J. Human genetic variation and the gut microbiome in disease. Nat. Rev. Genet. 18, 690-699 (2017).

89. Enattah, N. S. et al. Identification of a variant associated with adult-type hypolactasia. Nat. Genet. 30, 233-237 (2002).

90. Sheather, S. J. \& Jones, M. C. A reliable data-based bandwidth selection method for kernel density estimation. J. R. Stat. Soc. 53, 683-690 (1991).

91. Kenward, M. G. \& Roger, J. H. An improved approximation to the precision of fixed effects from restricted maximum likelihood. Comput. Stat. Data Anal. 53, 2583-2595 (2009)

92. Faith, J. J. et al. The long-term stability of the human gut microbiota. Science 341, 1237439 (2013)

93. Kolde, R. Pheatmap: pretty heatmaps. R Package Version 1.0.10. https:// CRAN.R-project.org/package=pheatmap (2012).

94. Gibbons, R. D., Hedeker, D. \& DuToit, S. Advances in analysis of longitudinal data. Annu. Rev. Clin. Psychol. 6, 79-107 (2010).

95. Minot, S. et al. The human gut virome: inter-individual variation and dynamic response to diet. Genome Res. 21, 1616-1625 (2011).

96. Shannon, P. et al. Cytoscape: a software environment for integrated models of biomolecular interaction networks. Genome Res. 13, 2498-2504 (2003).

97. Gonzalez, A. et al. Qiita: rapid, web-enabled microbiome meta-analysis. Nat Methods 15, 796-798 (2018)

98. Edgar, R., Domrachev, M. \& Lash, A. E. Gene Expression Omnibus: NCBI gene expression and hybridization array data repository. Nucleic Acids Res. 30 , 207-210 (2002)

99. Vizcaíno, J. A. et al. 2016 update of the PRIDE database and its related tools. Nucleic Acids Res. 44, D447-D456 (2016)

100. Suzek, B. E., Huang, H., McGarvey, P., Mazumder, R. \& Wu, C. H. UniRef: comprehensive and non-redundant UniProt reference clusters. Bioinformatics 23, 1282-1288 (2007).

101. Bar-Joseph, Z., Gifford, D. K. \& Jaakkola, T. S. Fast optimal leaf ordering for hierarchical clustering. Bioinformatics 17 (Suppl. 1), S22-S29 (2001).

102. Silverman, B. W. Density Estimation for Statistics and Data Analysis 48, eqn 43.31 (Chapman and Hall, 1986).

103. Wellcome Trust Case Control Consortium. Genome-wide association study of 14,000 cases of seven common diseases and 3,000 shared controls. Nature 447, 661-678 (2007) 


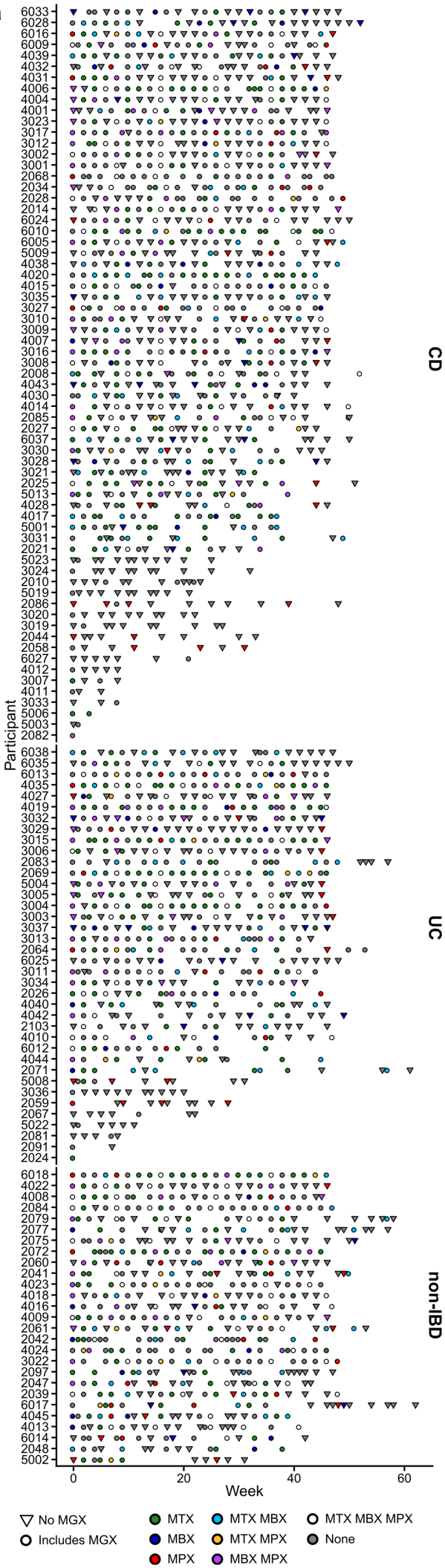

b

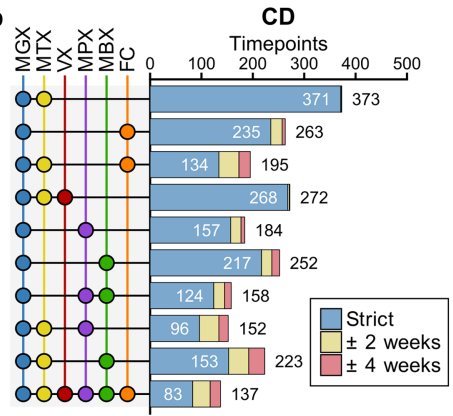

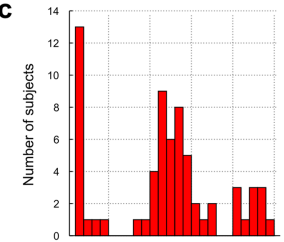
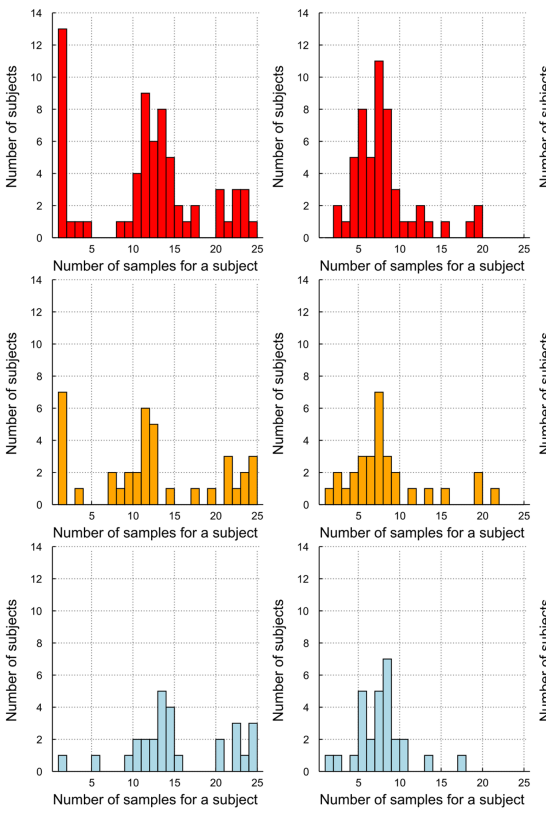

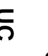

d
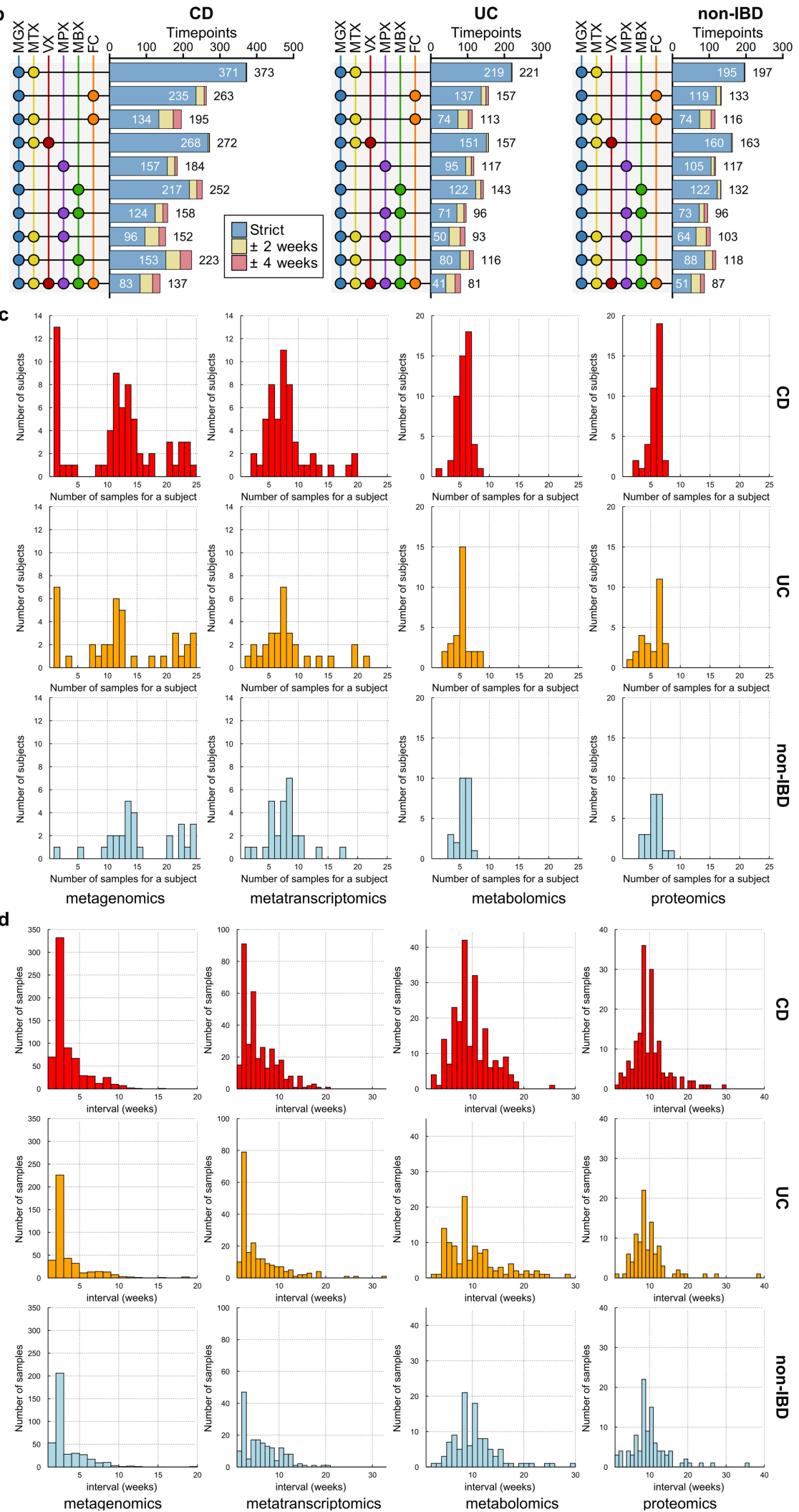

Extended Data Fig. 1 | Distribution of sample and measurement types and timing. a, Measurements available over time for each IBDMDB participant. $\mathbf{b}$, Number of identical or closely-aligned time points available for which each measurement type has been generated (see Methods). c, Distributions of the number of processed samples per subject, stratified by disease and measurement type. d, Distributions of time intervals between consecutive samples for each measurement type. 

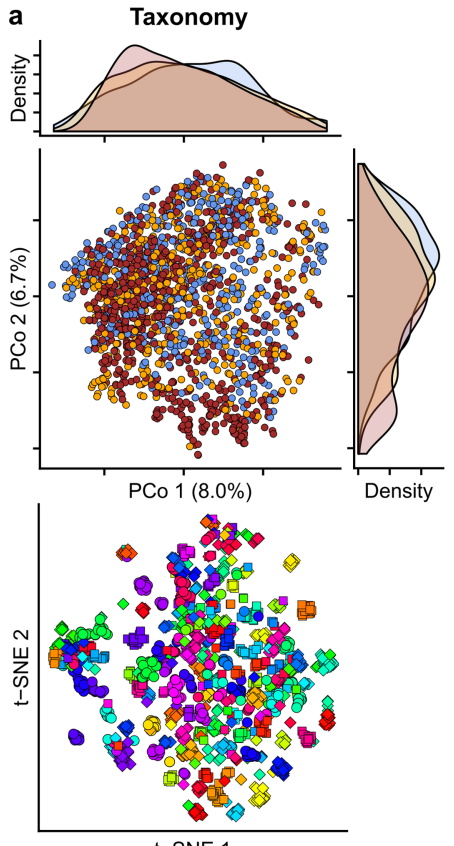

t-SNE 1

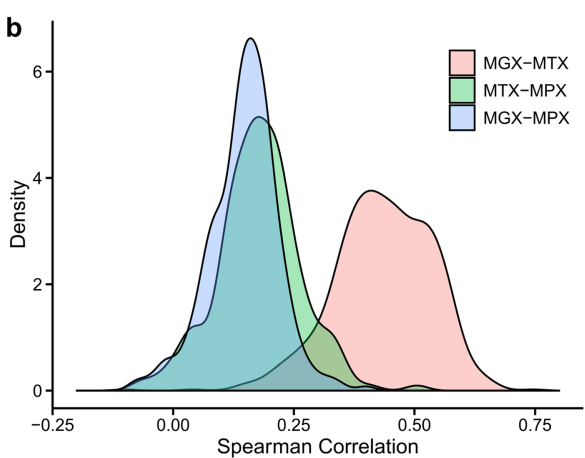

Extended Data Fig. $2 \mid$ Within-individual stability is a major driver of microbiome differences across measurement types. a, PCoA and $\mathrm{t}$-SNE embeddings based on Bray-Curtis dissimilarity matrices from stool species abundances, transcripts, proteins, and metabolites. Marginal densities are shown for the PCoAs that show disease separation for some measurements. In the t-SNEs, each subject has been assigned a different hue, showing that small clusters generally represent individuals' time courses, as inter-individual differences are the greatest driver of microbiome variation across measurement types (Fig. 1f). Sample counts are shown in Fig. 1b. b, Distributions of correlations between functional profiles, captured as UniRef $90^{100}$ gene family abundances ${ }^{73}$,
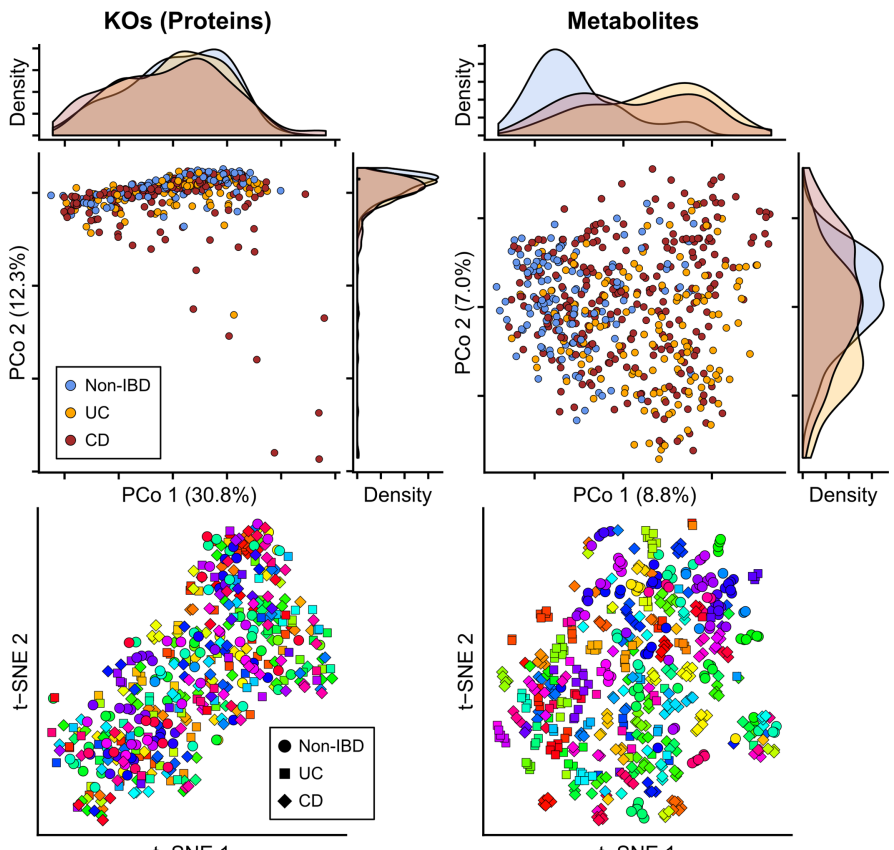

t-SNE 1
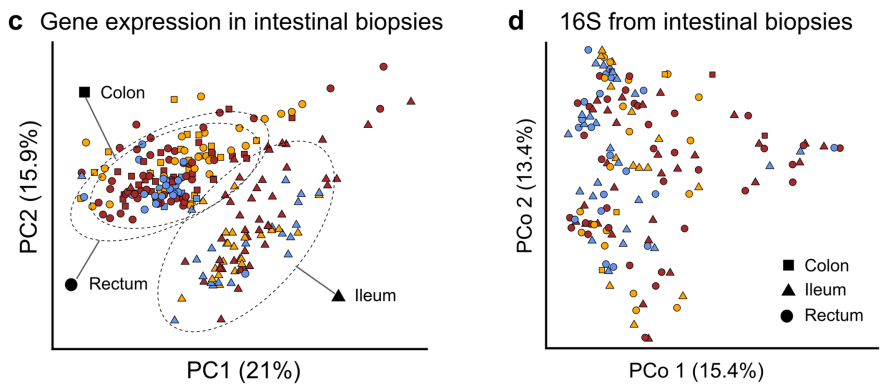

measured from paired metagenomes, metatranscriptomes, and metaproteomes (see Methods). c, Human transcriptional expression was mostly determined by biopsy location rather than IBD phenotype and inflammation ( $n=249$ samples from 91 subjects). Ordination shows PCA on gene expression levels normalized by library sizes and represented as CPM. Ellipses indicate $95 \%$ confidence regions for the indicated sample types. d, Principal coordinates plot (Bray-Curtis on OTU profiles) of community profiles from biopsy samples shows that mucosal microbial communities do not differ significantly by biopsy location (shape), unlike human gene expression in epithelial tissue (Fig. 4b). 


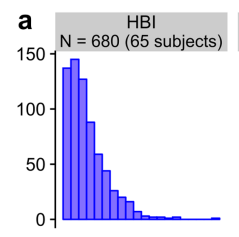

NA

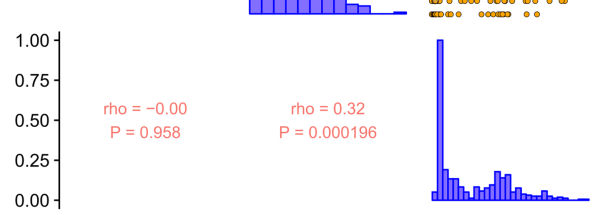

$0.00-1$

1.00
0.75

0.50
0.25

0.25
0.00

$0.00-$

1.00
0.75

0.50

0.25

$0.00-1$

$1.00-1$
0.75

0.5

0.25

$0.000 .250 .500 .751 .00 .000 .250 .500 .751 .00 .000 .250 .500 .751 .00 .000 .250 .500 .751 .00 .000 .250 .500 .750 .1 \%$

Extended Data Fig. 3 | Patient-reported, molecular, and microbial disease activity measures. a, Relationships between six measures of disease activity: the patient-reported $\mathrm{HBI}$ in $\mathrm{CD}$, patient-reported SCCAI in UC, faecal calprotectin, the fractions of human reads from stool MGX and MTX, and a dysbiosis score defined here as departures from control population microbiome configurations (Fig. 2c). Rho values are Spearman correlations with ties broken randomly. Linear regression is shown (red line) with $95 \%$ confidence bound (shaded). Sample counts are presented in the title bar, though sample counts for a particular correlation may be less as samples must be paired. b, c, PCoA based on metagenomic species-
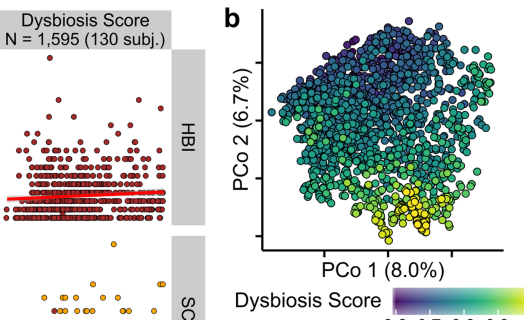$$
\text { Dyst }
$$
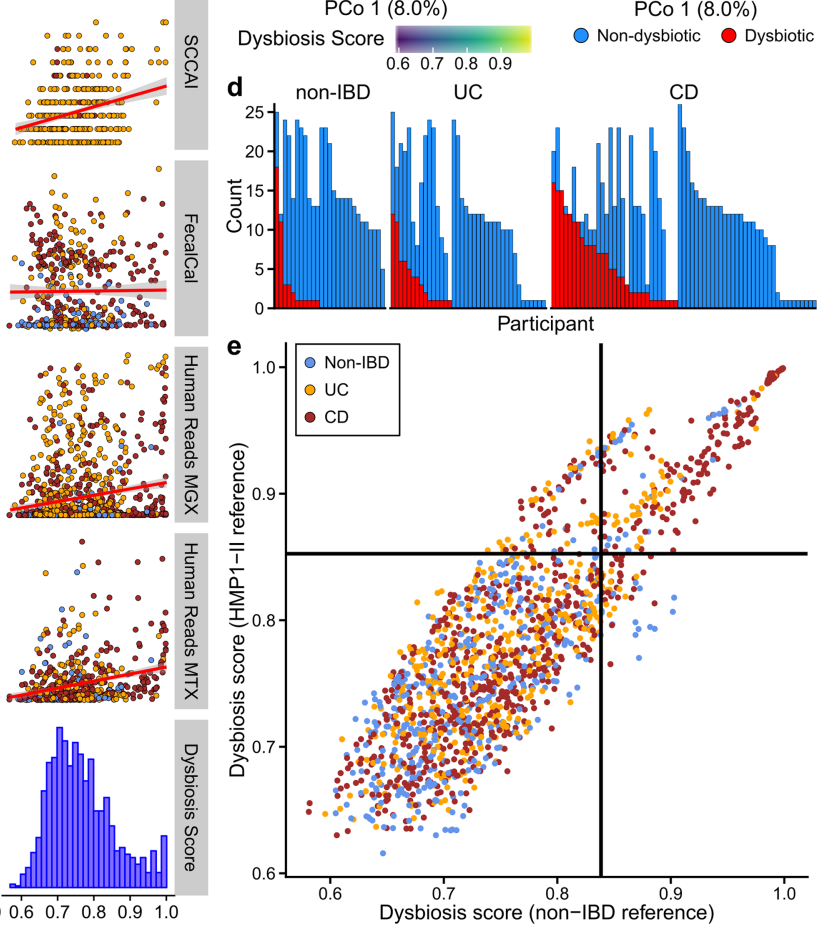

level Bray-Curtis dissimilarities ( $n=1,595$ samples from 130 subjects), indicating dysbiosis score (b) and whether the sample was defined as dysbiotic (c). d, Number of dysbiotic samples per participant. Colour scheme as in c. e, Relationship between the dysbiosis score, when using the HMP1-II gut data set as reference ( $n=553$ from 249 subjects), compared to the non-IBD data set. The threshold for the dysbiosis classification is also shown (black lines). The two scores are highly correlated (Pearson $\rho=0.86$; two-sided $P<2.2 \times 10^{-16}$ ), as are the resulting dysbiosis classifications (odds ratio of 56; Fisher's exact test $P<2.2 \times 10^{-16}$ ). $n=1,595$ samples from 130 subjects. 
a
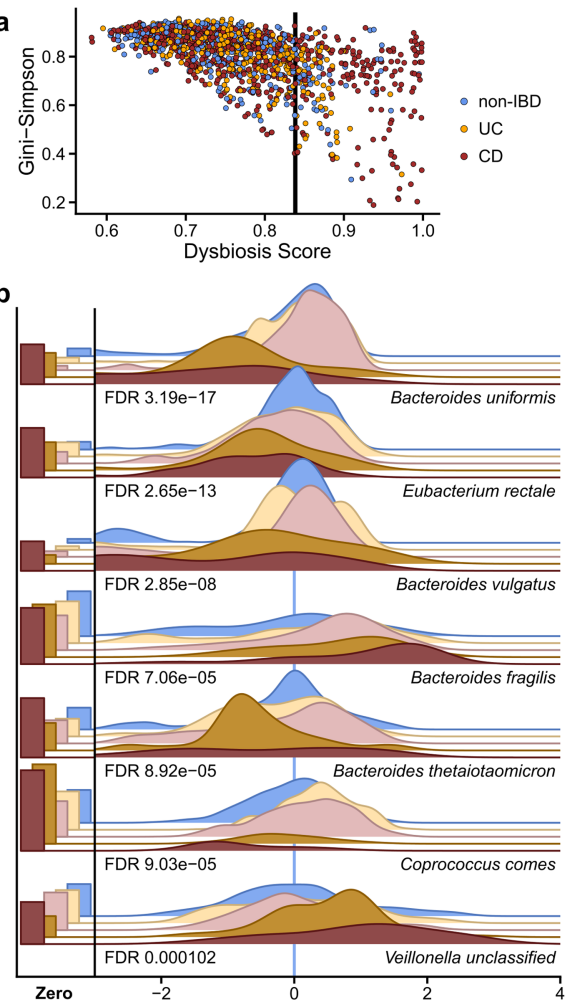

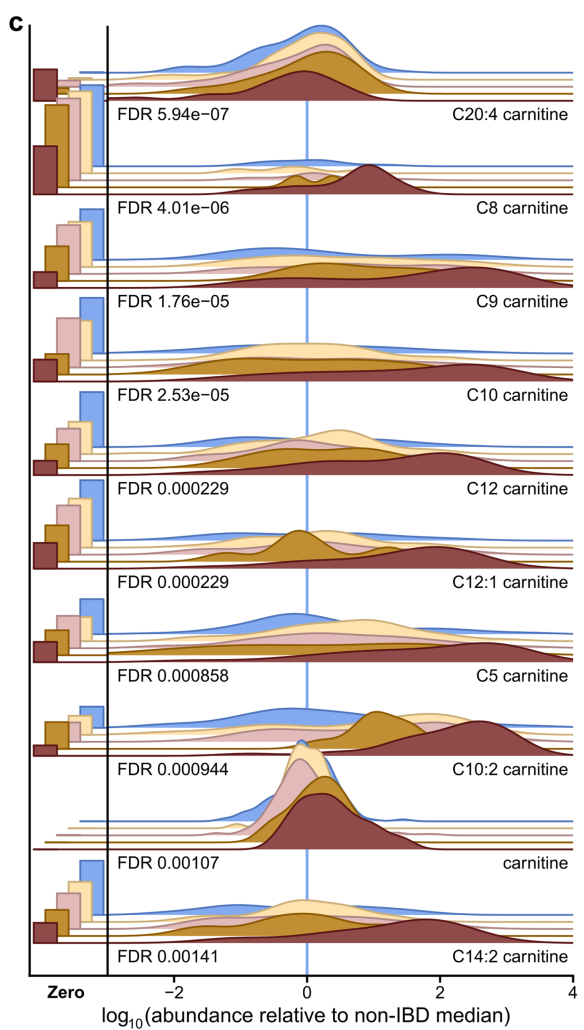

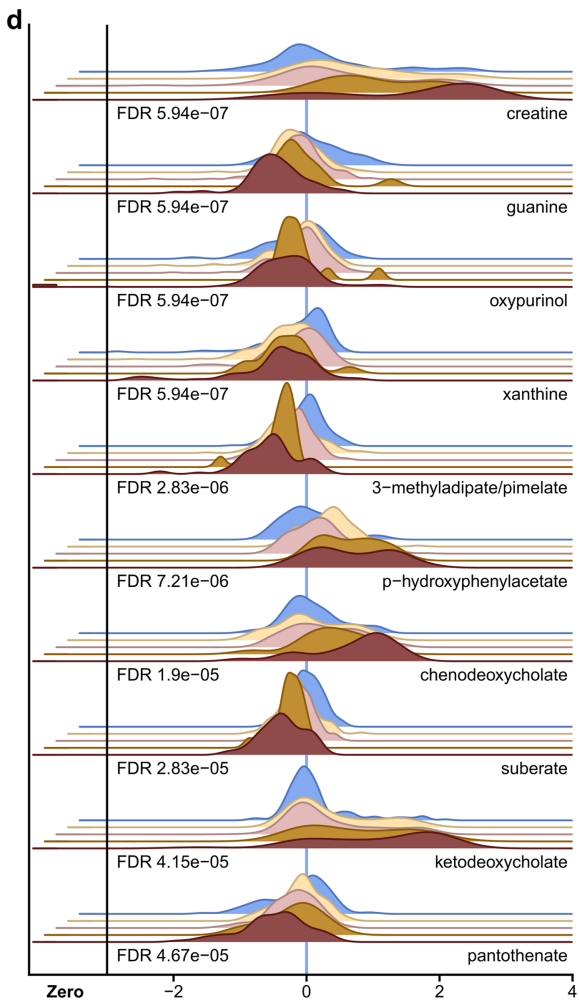

Extended Data Fig. 4 | Significant microbial and metabolic perturbations during taxonomic dysbioses. a, Alpha diversity (Gini-Simpson) as a function of the dysbiosis score for the sample (Pearson correlation $\left.-0.60 ; P<2.2 \times 10^{-16}\right) . n=1,595$ samples from 130 subjects. b, Seven (chosen for space constraints) most differentially abundant species not shown elsewhere in this manuscript $(n=1,595$ samples from 130 subjects; Wald test; see Methods; full results in
Supplementary Table 15). c, Top 10 differentially abundant acylcarnitines in dysbiosis ( $n=546$ samples from 106 subjects; Wald test). Carnitine and acylcarnitines are more abundant in dysbiotic CD, whereas C20:4 carnitine is significantly depleted (Supplementary Table 16). d, Top 10 differentially abundant metabolites during dysbiosis not shown elsewhere in this manuscript ( $n=546$ samples from 106 subjects; Wald test; full results in Supplementary Table 16). 
a

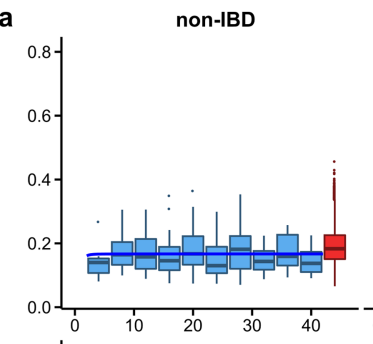

uc

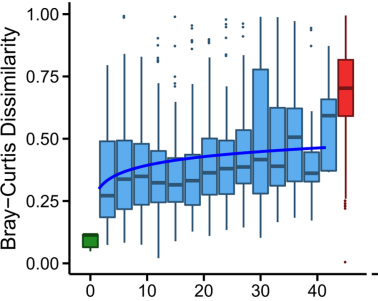

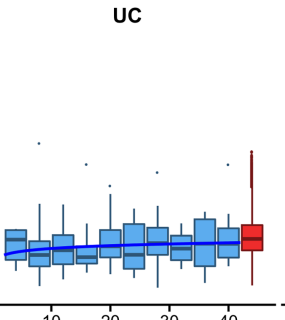
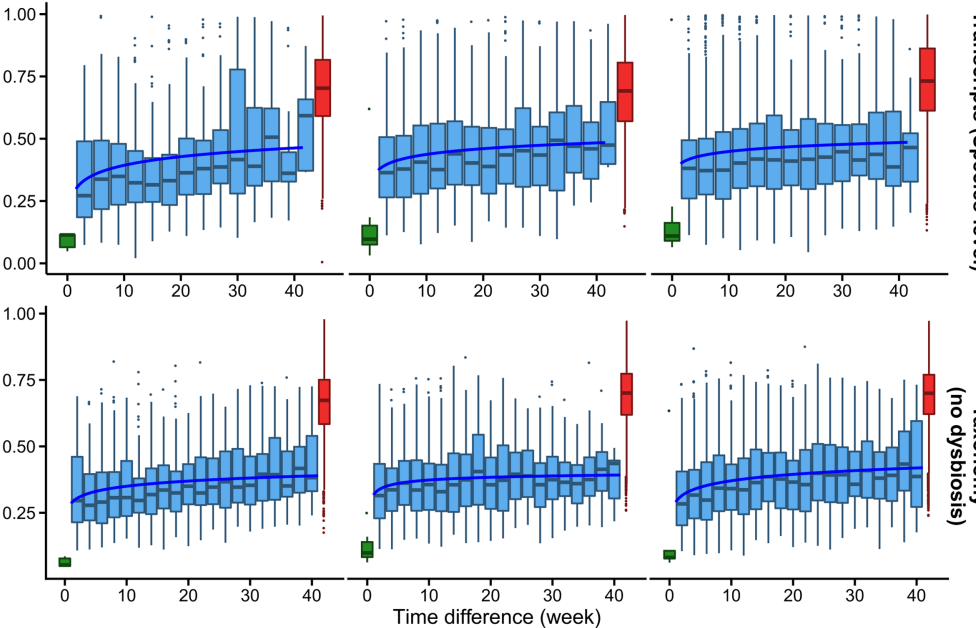

Time difference (week)
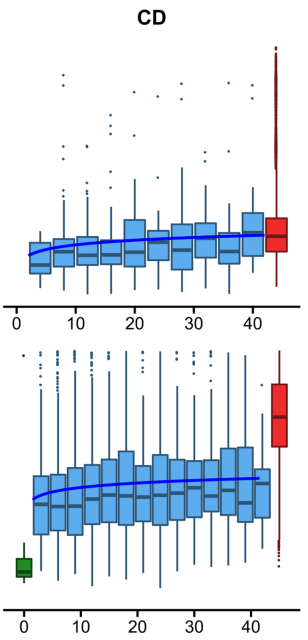

C
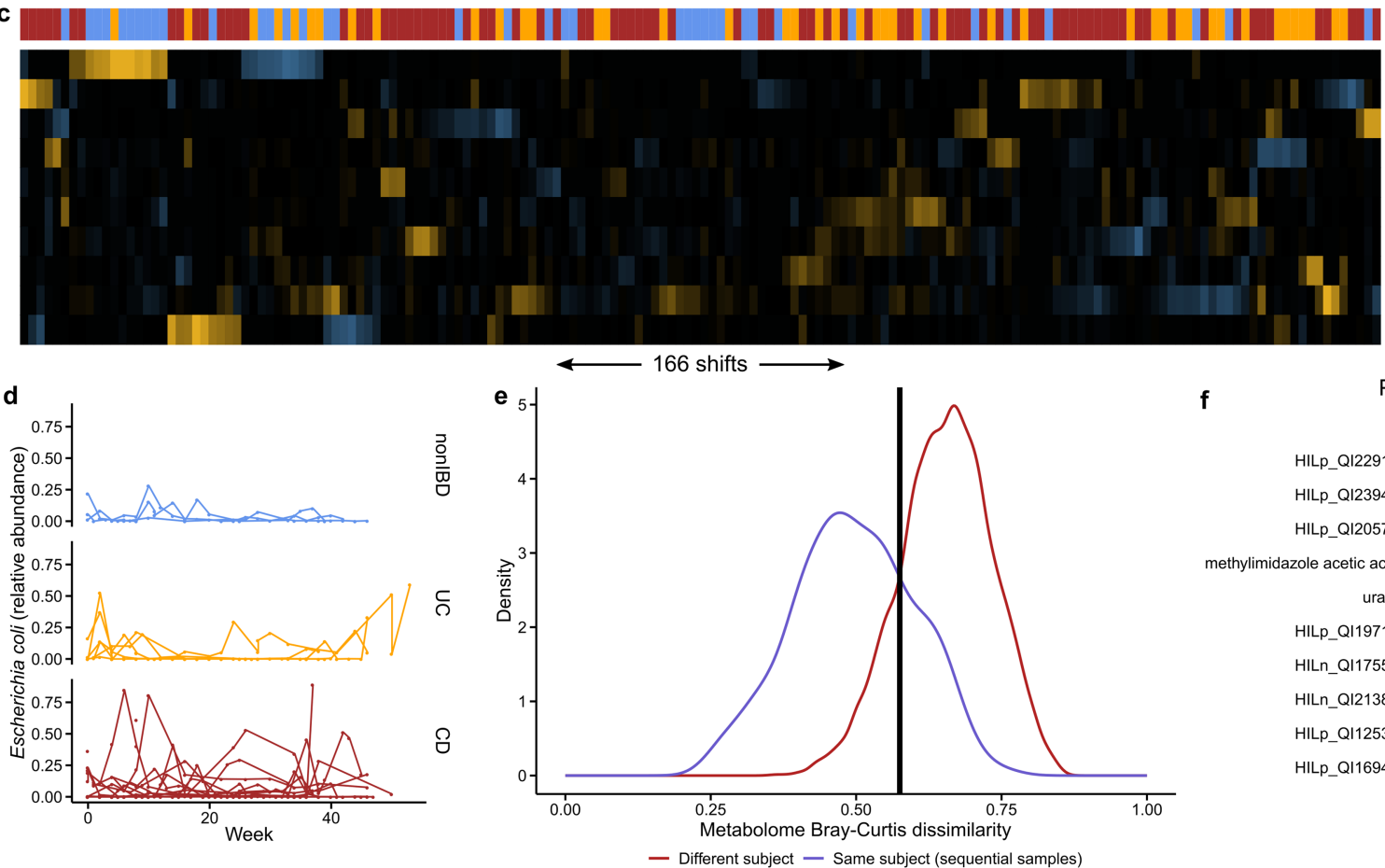

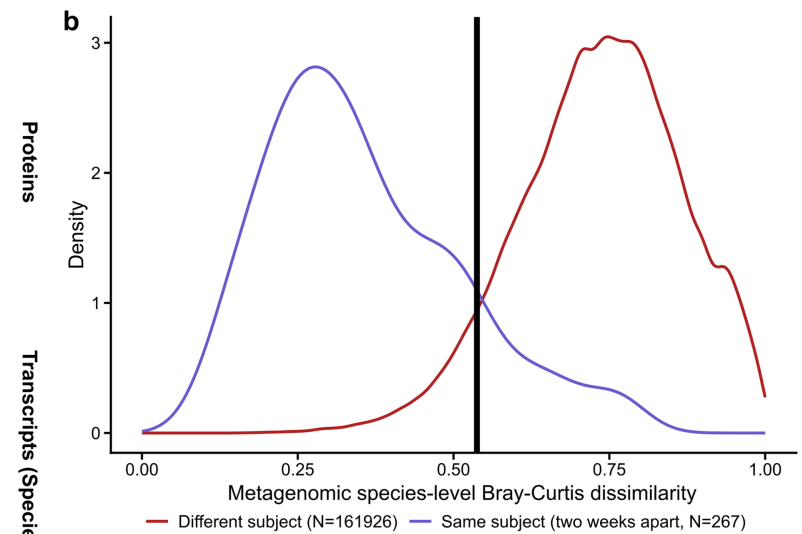

non-IBD UC $\square$ CD $-0.5$ 0 Relative abundance difference in shift Diagnosis Prevotella copri Escherichia coli Bacteroides fragilis Bacteroides vulgatus Bacteroides ovatus Bacteroides stercoris Bacteroides uniformis Eubacterium rectale Faecalibacterium prausnitzii Roseburia intestinalis

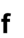

Primary contributors to metabolomic shifts

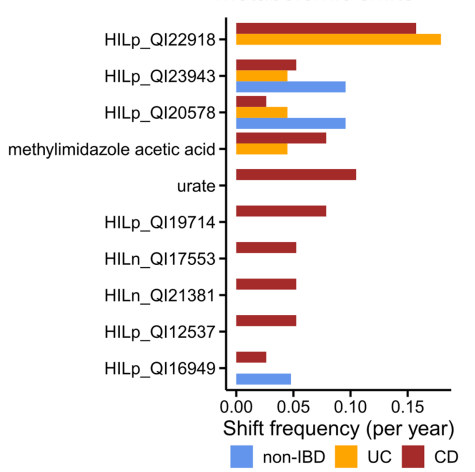

Extended Data Fig. 5 | See next page for caption. 
Extended Data Fig. 5 Detecting shifts in longitudinal microbiome multi-omics. a, Distributions of Bray-Curtis dissimilarities as a function of time difference between samples for protein profiles, species-level transcriptional activity (see Methods), and species-level taxonomy (though excluding subjects with dysbiosis at any time point), otherwise as in Fig. 3a. Removing subjects with dysbiotic samples removes the extreme dissimilarities (near 1) observed in IBD subjects. Boxplots show median and lower/upper quartiles; whiskers show inner fences. b, Distribution of Bray-Curtis dissimilarities between samples from the same subject, two weeks apart versus those from different individuals, allowing us to define a 'shift' in the microbiome as a change more likely to have been drawn from the between-subject distribution than within-subject distances (corresponding to Bray-Curtis > 0.54). c, Relative abundance differences of the top ten microorganisms that contributed to each of the 183 detected taxonomic shifts among any two within-subject subsequent time points. Shifts are typically reciprocal (that is, losing a microorganism and regaining it later, or vice versa), and microorganism with frequent high-abundance shifts generally correspond to frequent contributors in Fig. 3b. Sample ordering is from a hierarchical clustering using average linkage followed by optimal leaf ordering ${ }^{101}$. d, As in Fig. 3c, but for E. coli
( $n=322$ samples from 24 subjects; two-tailed Wilcoxon test of the absolute differences in relative abundances between consecutive time points $P=2.2 \times 10^{-4}$ for non-IBD to UC, and $P=0.029$ for non-IBD to $\mathrm{CD}$ ), which is frequently implicated in gut inflammation. e, As in b, but showing Bray-Curtis dissimilarities of metabolomic profiles. Here, $22 \%$ (96 out of 440 ) of sample pairs exceed the shift threshold, whereas $13 \%$ (183 out of 1,413$)$ exceed the threshold in $\mathbf{b}$. If metagenomic profiles are sub-sampled to match the metabolomics samples, this increases to $14 \%$ (57 out of 398 ) of sample pairs, showing that if we increased the sampling rate, this measurement type would be likely to shift more than the metagenomes. $\mathbf{f}$, As in Fig. 3b, but showing the primary contributors to metabolomic shifts, that is, the metabolite with the largest change in relative abundance during a shift. Note that other metabolites may still experience large changes in abundance (for example, for this reason, urate was not a primary contributor to any non-IBD shifts, though large changes are visible for one non-IBD individual in Fig. 3e). The full table of detected metabolomic shifts is given in Supplementary Table 30. Violin plot shows the density of points around that intake frequency; bandwidth chosen automatically by Silverman's method ${ }^{102}$. 
a

\begin{tabular}{ccccc} 
& & \multicolumn{3}{c}{ Intestinal biopsies } \\
& & lleum & Colon & Rectum \\
\hline \multirow{2}{*}{ CD } & inflamed & 43 & 15 & 47 \\
\cline { 2 - 5 } & non-inflamed & 0 & 13 & 1 \\
\hline \multirow{2}{*}{ UC } & inflamed & 0 & 13 & 26 \\
\cline { 2 - 5 } & non-inflamed & 24 & 9 & 0 \\
\hline & non-IBD & 21 & 6 & 23 \\
\hline
\end{tabular}

b
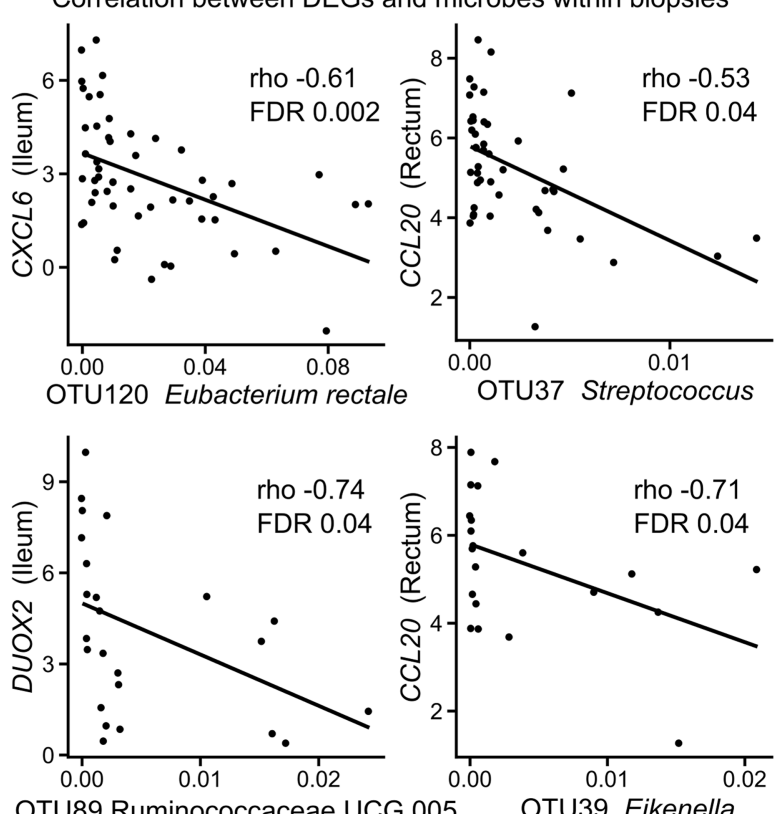

OTU89 Ruminococcaceae UCG 005

Extended Data Fig. 6 | Mucosal communities and human genetics.

a, Number of biopsy samples available for each biopsy location and inflammation status. b, DEGs (Fig. 4a) with newly identified significant correlations with OTU abundances in biopsies (partial Spearman correlation conditioned on disease status, BMI, age at consent and sex; FDR $P<0.05 ; n=54$ in ileum and $n=52$ independent 16S-RNA-seq pairs; full table in Supplementary Table 33). c, A limited subset of the microbiome trended with genetic variants in targeted testing, including the strongest trend shown here of Parabacteroides distasonis with genotypes of NKX2-3 (a known IBD-associated locus ${ }^{103}$; boxplots show median and lower/upper quartiles; whiskers show inner fences). This is the most significant association by $P$ value among all tested associations between metagenomic taxa and five known IBD loci (nominal significance $P=0.006$; no associations passed FDR $P<0.05$, mixed effect model with age, sex, antibiotic and immunosuppressant use and first 20 genetic principal components as covariates while specifying subjects as random
C
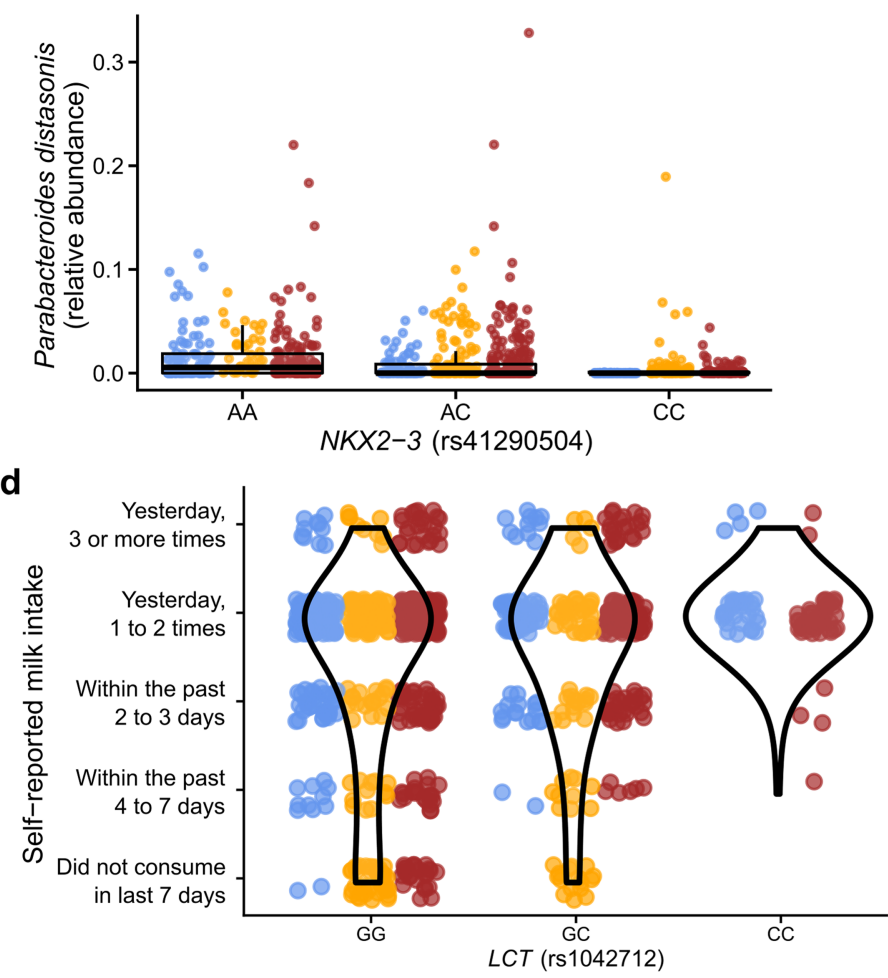

effects; Wald test; $n=84$ subjects of European ancestry with exomes and 960 metagenomes; full results in Supplementary Table 34). d, Association between rs1042712 SNP in the LCT locus and self-reported milk intake from dietary recall. Self-reported short-term milk intake (from dietary recalls accompanying stool samples) was significantly associated with the count of C alleles ( $29.8 \%$ allele frequency) at rs 1042712 in the LCT gene locus using a linear mixed effect model accounting for age, sex, first 20 genetic principal components and with subjects as random effects ( $P=0.028$, linear mixed effect regression with Wald test, see Methods). All available data are plotted for unique subjects of European ancestry with exome data (per-genotype subject count (GG/GC/CC):50/26/8). Differences between IBD and non-IBD groups are not statistically significant (odds ratio $0.27 ; 95 \%$ CI $0.05-1.33 ; P=0.10 ; n=84$ subjects of European ancestry with exomes and 960 dietary surveys; model: IBD (yes|no) $\sim$ intercept + SNP + sex + age + PC1-PC20). 
a

Subdoligranulum, Faecalibacterium, and Roseburia sub-networks

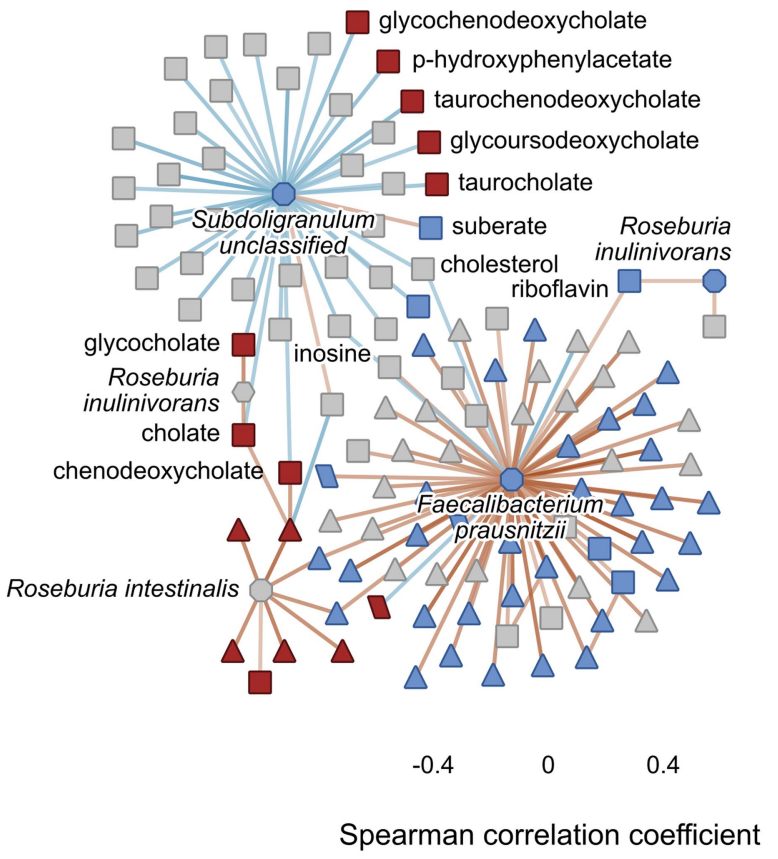

Extended Data Fig. 7 | Microbial and host-related subsets of the multiomic association networks. a, Subset of the network in Fig. 4c showing metagenomic abundances (octagons) and expression levels (hexagons) of Subdoligranulum, Roseburia spp. and F. prausnitzii and their neighbours (three functionally associated microbial hubs selected for further b Host-related subnetwork (unadjusted)

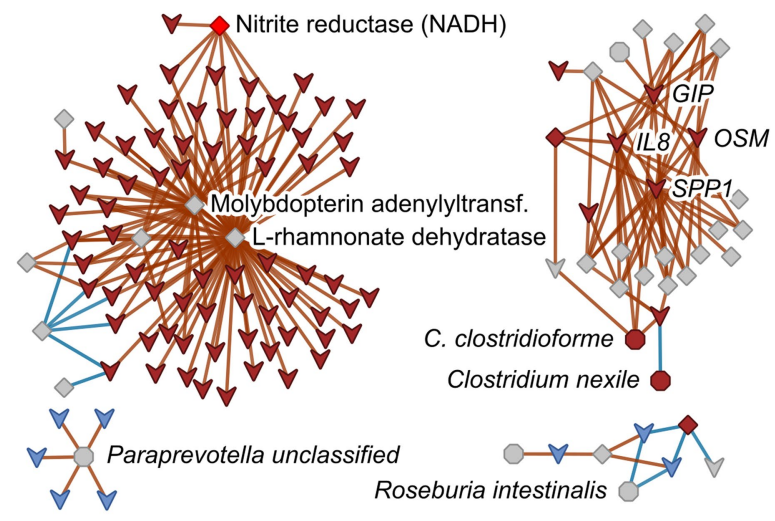

$\begin{array}{ll} & \bigcirc \text { Species (MGX) } \\ & \square \text { Species (MTX/MGX) } \\ & \bigcirc \text { EC (MGX) } \\ \text { Unchanged in dysbiotic IBD } & \triangle \text { EC (MTX) } \\ \text { Down in dysbiotic UC or CD } & \square \text { EC (MPX) } \\ \square \text { Up in dysbiotic UC } & \square \text { Metabolite } \\ \text { Up in dysbiotic CD } & \square \text { DEG (HTX) } \\ \text { Up in dysbiotic UC and CD } & \bigcirc \text { Sera/Calprotectin }\end{array}$

investigation based on anti-inflammatory associations in the literature, see text). b. The host expression-related subnetwork of the 'unadjusted' association network (Extended Data Fig. 9, Supplementary Discussion). Sample counts in Fig. 1b, c. 


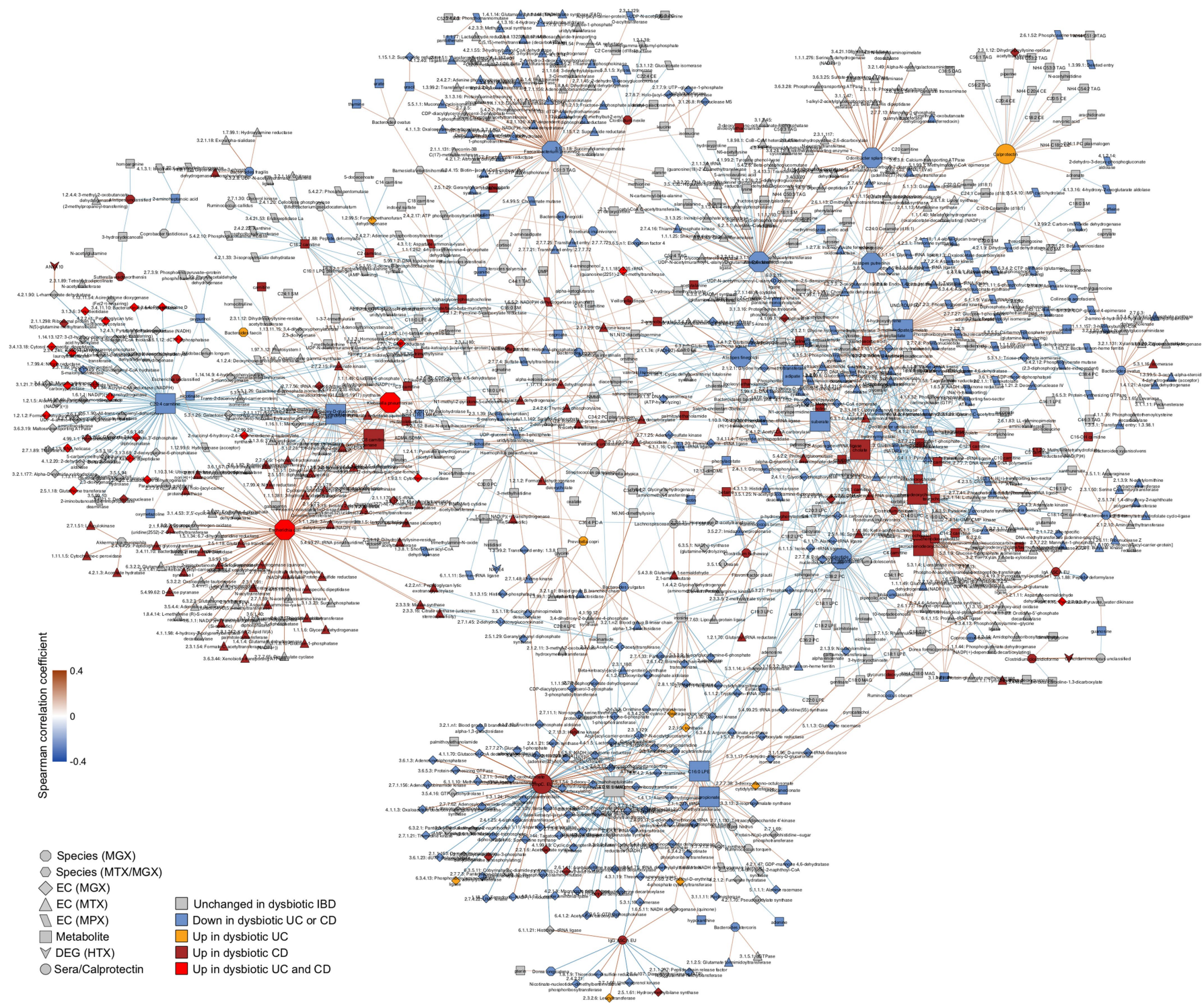

Extended Data Fig. 8 | Significant covariation among multi-omic components of the gut microbiome and host interactors in IBD (adjusted). Detailed labelling of the association network in Fig. 4c (intended for magnification). The network was constructed from ten data sets: metagenomic species, species-level transcription ratios, functional profiles at the EC levels (MGX, MTX and MPX), metabolites, host transcription (rectal and ileal separately), serology and faecal calprotectin. As in Fig. 4c, measurement types were approximately matched in time with a maximum separation between paired samples of four weeks. The top 300 significant correlations (FDR $P<0.05$ ) among correlations between features that were differentially abundant in dysbiosis were used to construct the network visualized here (for serology, a threshold of FDR $P<0.25$ was used). Nodes are coloured by the disease group in which they are 'high', and edges are coloured by the sign and strength of the correlation. For this adjusted network, Spearman correlations were calculated using HAllA from the residuals of a mixed-effects model with subjects as random effects (or a simple linear model without the random effects when only baseline samples were used) after adjusting for age, sex, diagnosis, dysbiosis status, recruitment site, and antibiotics (see Methods). Appropriate normalization and/or transformation for each measurement type was performed independently before the model fitting (see Methods). Singleton node pairs were pruned from the network. Source associations are in Supplementary Table 35, sample counts in Fig. 1b, c. 


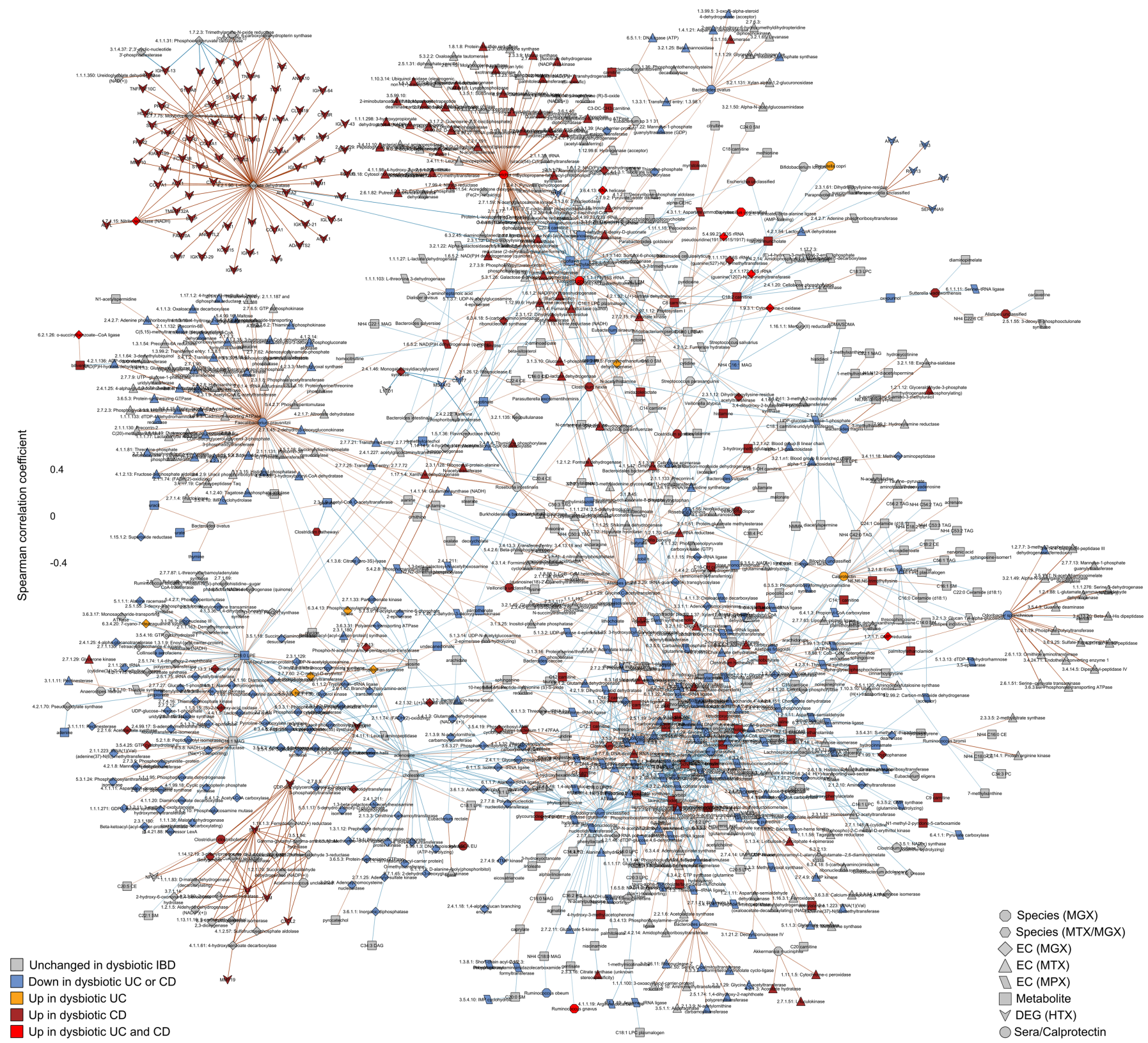

Extended Data Fig. 9 | Significant covariation among multi-omic components of the gut microbiome and host interactors in IBD

(unadjusted). The network was constructed from ten data sets: metagenomic species, species-level transcription ratios, functional profiles at the EC levels (MGX, MTX and MPX), metabolites, host transcription (rectal and ileal separately), serology and faecal calprotectin. As in Fig. 4c, measurement types were approximately matched in time with a maximum separation between paired samples of four weeks. The top 300 significant correlations (FDR $P<0.05$ ) among correlations between features that were differentially abundant in dysbiosis were used to construct the network visualized here (for serology, a threshold of FDR $P<0.25$ was used). Nodes are coloured by the disease group in which they are 'high', and edges are coloured by the sign and strength of the correlation. For this unadjusted network, Spearman correlations were calculated using HAllA from the residuals of the same model as in Extended Data Fig. 8, though without adjusting for dysbiosis (see Methods). Appropriate normalization and/or transformation for each measurement type was performed independently before the model fitting (see Methods). Singleton node pairs were pruned from the network. Source associations are in Supplementary Table 36, sample counts in Fig. 1b, c. 


\section{RESEARCH ARTICLE}

Extended Data Table 1 | IBDMDB cohort characteristics

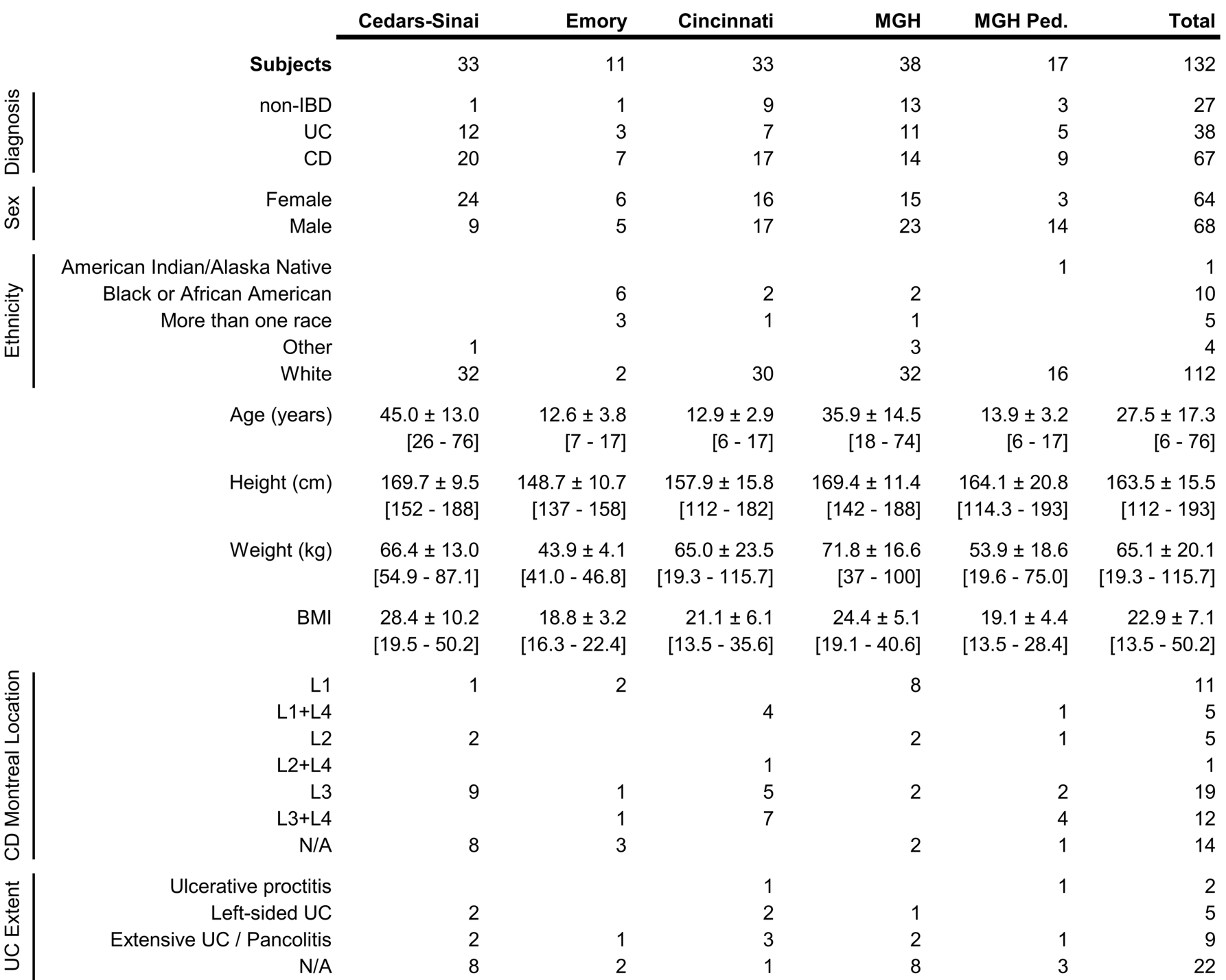

Breakdown of the cohort by diagnosis, sex, ethnicity, physical characteristics, and disease location for each clinical site. Numeric data are summarized as mean \pm s.d., with the range given by [min-max], and with blank cells for NA (not applicable). Subject counts for disease locations are subsets of the respective diseases. 


\section{Life Sciences Reporting Summary}

Nature Research wishes to improve the reproducibility of the work that we publish. This form is intended for publication with all accepted life science papers and provides structure for consistency and transparency in reporting. Every life science submission will use this form; some list items might not apply to an individual manuscript, but all fields must be completed for clarity.

For further information on the points included in this form, see Reporting Life Sciences Research. For further information on Nature Research policies, including our data availability policy, see Authors \& Referees and the Editorial Policy Checklist.

Please do not complete any field with "not applicable" or n/a. Refer to the help text for what text to use if an item is not relevant to your study. For final submission: please carefully check your responses for accuracy; you will not be able to make changes later.

\section{- Experimental design}

\section{Sample size}

Describe how sample size was determined.

\section{Data exclusions}

Describe any data exclusions.

\section{Replication}

Describe the measures taken to verify the reproducibility of the experimental findings.

\section{Randomization}

Describe how samples/organisms/participants were allocated into experimental groups.

\section{Blinding}

Describe whether the investigators were blinded to group allocation during data collection and/or analysis.
The target sample size calculated for at least $n=72$ subjects with repeated measures was designed to have power of 0.9 to detect 1 ) between-group differences in taxon abundance (repeated measures ANOVA, group F > 0.4), 2) differentially expressed transcripts (Edland's test for a linear mixed model with random slope, $d>0.07$ ), and 3 ) multi'omic correlations (Pearson correlation, $r>0.6$ ). Power calculations incorporated conservative Bonferroni $p$ value correction, with numbers of post-QC microbial features and within-sample correlations estimated from previous microbiome studies.

Potential subjects were excluded from the study if they were unable or did not consent to provide tissue, blood, or stool, were pregnant, had a known bleeding disorder or an acute gastrointestinal infection, were actively being treated for a malignancy with chemotherapy, were diagnosed with indeterminate colitis, or had a prior, major gastrointestinal surgery such as an ileal/colonic diversion or j-pouch. These criteria were established prior to the study start. Samples were filtered based on data type-specific quality control measures. For metagenomes and metatrascriptomes, samples were required to have $>1 \mathrm{M}$ reads and at least one species detected by MetaPhIAn2.

The study was a large-scale clinical cohort and we did not attempt to replicate all aspects of sample collection and data generation. However, data and source code for computational tools used are available to the public and therefore all of our analysis can be reproduced using our methods or re-analyzed using other methods. When possible, we refer to existing literature that supports our findings. Multiple pilot studies as well as technical replicates covering a subset of samples are also available, and these data were successfully integrated into subsequent multi-batch analyses, ensuring that data generation methods produced reproducible results.

Experimental groups could not be randomized as they depended on diagnosis. Participants were recruited into the three disease groups as available from each of the recruitment sites. Upon enrollment, an initial colonoscopy was performed to determine study strata. Subjects not diagnosed with IBD based on endoscopic and histopathologic findings were classified as "non-IBD" controls, including the aforementioned healthy individuals presenting for routine screening, and those with more benign or non-specific symptoms. This creates a control group that, while not completely "healthy", differs from the IBD cohorts specifically by clinical IBD status.

Samples were collected by clinical staff who were not blinded as they needed to examine patients to determine which experimental group they should be allocated to. All data were generated by investigators that were blinded to the metadata. Once data were generated, computational analysis was performed with all of the necessary clinical information to test between groups. 


\section{Statistical parameters}

For all figures and tables that use statistical methods, confirm that the following items are present in relevant figure legends (or in the Methods section if additional space is needed).

n/a Confirmed

$\bigotimes$ The exact sample size $(n)$ for each experimental group/condition, given as a discrete number and unit of measurement (animals, litters, cultures, etc.)

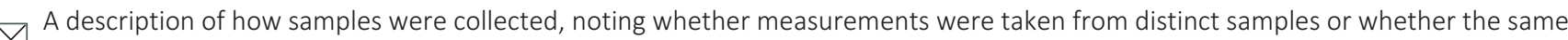

sample was measured repeatedly

Х $\square$ A statement indicating how many times each experiment was replicated

The statistical test(s) used and whether they are one- or two-sided

Only common tests should be described solely by name; describe more complex techniques in the Methods section.

$\bigotimes$ A description of any assumptions or corrections, such as an adjustment for multiple comparisons

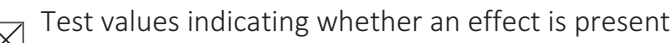

$\triangle$ Provide confidence intervals or give results of significance tests (e.g. P values) as exact values whenever appropriate and with effect sizes noted.

$\bigotimes$ A clear description of statistics including central tendency (e.g. median, mean) and variation (e.g. standard deviation, interquartile range)

$\bigotimes$ Clearly defined error bars in all relevant figure captions (with explicit mention of central tendency and variation)

See the web collection on statistics for biologists for further resources and guidance.

\section{- Software}

Policy information about availability of computer code

\section{Software}

Describe the software used to analyze the data in this study.

\author{
Analysis of mass spectra: MSGF+ software v10072 \\ Sequence processing: Picard 2.9.4 \\ Metagenome and metatranscriptome profiles generated using bioBakery meta'omics \\ workflow v0.9.0 (Preprocessing: KneadData 0.7.0; Taxonomic profiles: MetaPhlAn2 v2.6.0; \\ Functional profiles: HUMAnN2 v0.11.0 with UniRef release 2014_07, Diamond v0.8.22.84) \\ Viral profiles: VirMAP \\ 16S analysis: USEARCH v7.0.1090 \\ Data analysis and plotting: $R$ (main packages: edgeR, $n$ Ime, ggplot2, ggridges, pheatmap, \\ vegan, tsne), Julia plots package \\ Network construction and visualization: HAllA 0.8.17, Cytoscape 3.6.0
}

For manuscripts utilizing custom algorithms or software that are central to the paper but not yet described in the published literature, software must be made available to editors and reviewers upon request. We strongly encourage code deposition in a community repository (e.g. GitHub). Nature Methods guidance for providing algorithms and software for publication provides further information on this topic.

\section{- Materials and reagents}

Policy information about availability of materials

\section{Materials availability}

Indicate whether there are restrictions on availability of unique materials or if these materials are only available for distribution by a third party.

\section{Antibodies}

Describe the antibodies used and how they were validated for use in the system under study (i.e. assay and species).
Remaining biospecimen aliquots from the project are available by request from the corresponding author.

Antibodies were used for serology and the ELISAs we performed are not commercially available as kits or reagents (except for secondary antibodies). The primary antibodies are patient sera (validation not applicable).

Conjugated polyclonal secondary antibodies are utilized, clone not applicable: Jackson ImmunoResearch Laboratories goat anti-human IgG-alkaline phosphatase; Cat \# 109-056-098; 1:1000 dilution

Jackson ImmunoResearch Laboratories goat anti-human IgA-alkaline phosphatase; Cat \# 109-055-011; 1:1000 dilution 
10. Eukaryotic cell lines

a. State the source of each eukaryotic cell line used.

No eukaryotic cell lines were used.

b. Describe the method of cell line authentication used.

No eukaryotic cell lines were used.

c. Report whether the cell lines were tested for mycoplasma contamination.

d. If any of the cell lines used are listed in the database of commonly misidentified cell lines maintained by ICLAC, provide a scientific rationale for their use.

No eukaryotic cell lines were used.

No commonly misidentified cell lines were used.

\section{- Animals and human research participants}

Policy information about studies involving animals; when reporting animal research, follow the ARRIVE guidelines

\section{Description of research animals}

Provide all relevant details on animals and/or

No animals were used.

animal-derived materials used in the study.

Policy information about studies involving human research participants

12. Description of human research participants

Describe the covariate-relevant population

Population characteristics are presented in Extended Data Table 1.

characteristics of the human research participants. 